\title{
NBSIR 78-1483
}

\section{NBS: Properties of Electronic Materials}

J. R. Manning, R. L. Parker

National Bureau of Standards

Washington, D.C. 20234

Phone: (301) 921-3354

June 1978

Annua! Report

For the Period 1 April 1977 - 31 March 1978

NASA Goveroment Order H-27954B

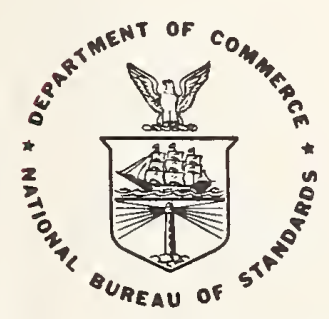

QC

U.S. DEPARTMENT OF COMMERCE 
NBSIR 78-1483

$\ldots$

NBS: PROPERTIES OF ELECTRONIC

MATERIALS

J. R. Manning, R. L. Parker

National Bureau of Standards

Washington, D.C. 20234

Phone: (301) 921-3354

June 1978

Annual Report

For the Period 1 April 1977 - 31 March 1978

NASA Government Order H-27954B

U.S. DEPARTMENT OF COMMERCE, Juanita M. Kreps, Secretary

Dr. Sidney Harman, Under Secretary

Jordan J. Baruch, Assistant Secretary for Science and Technology

NATIONAL BUREAU OF STANDARDS, Ernest Ambler, Director 
TABLE OF CONTENTS

$\underline{\text { Page }}$

Summary . . . . . . . . . . . . . 1-2

TaSk 1 - SURFACE TENSIONS AND THEIR VARIATIONS WITH TEMPERATURE AND IMPURITIES by

S. C. Hardy and S. R. Coriell.......... 3-14

Task 2 - SOLUTAL CONVECTION AND LIQUID DIFFUSION COEFFICIENTS by W. J. Boettinger, S. R. Coriell, F. S. Biancaniello and M. R. Cordes ............. 15-68

Task 3 - DETERMINATION OF CRYSTAL PERFECTION by M. Kuriyama and H. E. Burdette ......... 69-83

Task 4 - A THERMOCHEMICAL STUDY OF CORROSIVE REACTIONS IN OXIDE MATERIALS by H. S. Parker, R. S. Roth, C. D. Olson and E. R. Plante.............. 84-100

Task 5 - GRAVITY EFFECTS ON FLAME INHIBITION by J. W. Hastie ................ 101-111 Distribution List . . . . . . . . . . . . 112-113 
National Bureau of Standards

Properties of Electronic Materials

\section{Summary}

This report describes NBS work for NASA in support of NASA's Materials Processing in Space Program covering the period April 1, 1977 to March 31, 1978. The NBS program has two main thrusts:

1) Carrying out precision measurements in space and investigating the feasibility of improved measurements when the space environment offers a unique opportunity for performing such measurements. These measurements would be useful for either space processing or processes on the ground.

2) Obtaining precision measurements on materials properties when these properties are important to the design and interpretation of space processing experiments. These measurements would be carried out either in space or on the ground。

The NBS work is carried out in five tasks. These tasks have, as two of their focal points, the role of convection effects and the role of container effects, both of which would differ in space-based experiments from those found in ground-based experiments. The results obtained for each task are given in detailed summaries in the body of the report. Briefly, in Task I - Surface Tensions and their Variations with Temperature and Impurities - surface tension measurements have been made for gallium using the pendant drop technique and some complex 
effects ascribed to impurities were observed。In Task 2 - Solutal Convection and Liquid Diffusion Coefficients - the onset of convective and interfacial instabilities during the vertical directional solidification of a binary alloy has been determined theoretically by means of a linear stability analysis。 In Task 3 - Determination of Crystal Perfection - a study has been performed on the improvement in resolution of $x$-ray imaging systems for real-time assessment of crystal perfection. In Task 4 - A Thermochemical Study of Corrosion Reactions in Oxide Materials - the system $\mathrm{KFeO}_{2}-\mathrm{Fe}_{2} \mathrm{O}_{3}$ has been examined over portions of the subsolidus region, and the occurrence of a beta alumina structuretype at 85.2 mole percent $\mathrm{Fe}_{2} \mathrm{O}_{3}$ has been observed。In Task 5 - Gravity Effects on Flame Inhibition - a high pressure sampling mass spectrometer facility has been designed and construction on it is nearly completed 。 


\title{
Surface Tensions and Their Variations with Temperature and Impurities
}

\author{
S. C. Hardy and S. R. Coriell \\ Metal Science and Standards Division \\ Center for Materials Science
}

\begin{abstract}
Summary
We have measured the surface tension of gallium in ultra pure helium atmospheres at room temperature using the pendant drop technique and have observed a time dependence. This time dependence is more complex than that found in previous work. We have tentatively interpreted the observed time variation of the surface tension in terms of a bulk phase impurity which diffuses to the surface and desorbs. These preliminary investigations indicate the surface tension of pure gallium is near $800 \mathrm{~mJ} / \mathrm{m}^{2}$, a value about $10 \%$ higher than the equilibrium values previously reported.
\end{abstract}




\section{INTRODUCTION}

We have started an experimental and theoretical program to accurately measure the liquid-vapor surface tensions of metals and semiconductors. Accurate measurements of surface tension as a function of temperature and concentration would be of value in many industrial applications on earth and are essential in developing experiments and manufacturing processes for space which involve free liquid surfaces. The importance of surface tension related phenomena in microgravity is attested by a number of unpredicted and essentially not understood phenomena which have occurred in space experiments involving solidification.

Although technologically significant, the surface tensions of many metals and semiconductors are not well known and the temperature and concentration variations are uncertain. Indeed, even the qualitative variation of surface tension with temperature is in doubt with contradictory measurements showing surface tension both increasing and decreasing with temperature in some cases. Although fluid flow can be calculated from the relevant differential equations, such calculations require knowledge of the temperature and concentration dependence of the surface tension in order to be predictive.

Gallium was selected for the first measurements in this program because it has been suggested as a model material for ground based fluid flow studies in the NASA program, it is an intrinsically interesting metal, and its surface tension is known only approximately. In 
addition, it has a boiling point near $2200{ }^{\circ} \mathrm{C}$ and it can be supercooled by more than $100{ }^{\circ} \mathrm{C}$ below its $30^{\circ} \mathrm{C}$ melting point. Thus a very large temperature range is available for surface tension measurements.

There have been four previous studies of the surface tension of gallium. An early measurement by Richards and Boyer ${ }^{[1]}$ found a value of $358.2 \mathrm{~mJ} / \mathrm{m}^{2}$ in an atmosphere of carbon dioxide. This result is certainly very much lower than the value for pure gallium. Later measurements by Mack et al ${ }^{[2]}$ and Timofeevicheva and Pubachevich ${ }^{[3]}$ found surface tensions in the $700 \mathrm{~mJ} / \mathrm{m}^{2}$ to $750 \mathrm{~mJ} / \mathrm{m}^{2}$ range at temperatures near the melting point and under atmospheres of hydrogen and carbon dioxide. The most recent measurements by Abbaschian [4] also found values in this range with nitrogen atmospheres. However, he also observed a time dependence of the surface tension: the surface tensions of the pendant drops declined from over $800 \mathrm{~mJ} / \mathrm{m}^{2}$ initially to a constant value of about $725 \mathrm{~mJ} / \mathrm{m}^{2}$ in fifteen minutes. This terminal value is thus in agreement with earlier measurements. However, the time dependence of his observations suggests impurity adsorption with a consequent surface tension lowering. Thus it seems probable that all previous measurements of the gallium surface tension were strongly influenced by surface adsorption with the clean gallium value being over $800 \mathrm{~mJ} / \mathrm{m}^{2}$. The temperature variation of the surface tension derived from these measurements must be regarded as highly uncertain. 


\section{EXPERIMENTAL PROCEDURES}

The initial phase of this work has attempted to develop techniques for handling gallium which, by generating a clean surface, will produce an accurate surface tension value. Since the temperature variation of the surface tension can not profitably be studied until such a clean surface is obtained, all the measurements have been made at ambient temperature, i.e., $23^{\circ} \mathrm{C}$. Thus the gallium is undercooled by about 7

${ }^{\circ} \mathrm{C}$. Variations of several degrees in the ambient temperature will result in only very small surface tension variations since the temperature coefficients for metals are of the order of $0.1 \mathrm{~mJ} / \mathrm{m}^{2} \mathrm{~K}$.

The pendant drop technique was used for the measurements, the gallium drops being formed at the end of a micrometer syringe with a pyrex-glass tip of about $1.25 \mathrm{~mm} \mathrm{i.d.} \mathrm{The} \mathrm{teflon} \mathrm{plunger} \mathrm{of}$ syringe passed through an 0-ring which formed an air tight seal and the syringe was inserted through a vacuum coupling into a glass cell with flat faces. The cell-syringe assembly was checked for leaks by pressurizing to several psi and immersing in water to detect bubbles.

The gallium was obtained in 1 gm units in plastic ampoules and was nominally $99.9999 \%$ pure. The ampoules were filled with alcohol containing a little hydrochloric acid and slightly warmed on a hot plate to melt the solid gallium and to form a compact drop at the bottom of the ampoule. The contents of several ampoules were then drawn into the micrometer syringe which had been previously filled with alcohol. The gallium was rinsed at least six times with absolute ethyl alcohol 
within the syringe to eliminate all acid. The alcohol during this procedure was kept vigorously boiling to minimize dissolved gases.

The cell prior to and during the measurements was continuously flushed with helium gas purified by an instrument which uses titanium metal at $800{ }^{\circ} \mathrm{C}$ to remove impurities. The manufacturer of this device claims titanium gettering reduces oxygen pressures to about $10^{-22}$ atmospheres with similar levels for carbon and nitrogen. These are impurity levels lower than those found in ultra high vacuum, i.e., $10^{-16}$ atmosphere. However, gases desorbed from the tubulation to this device and from the cell are probably far above this level.

Several drops of gallium were formed and discarded before retaining one for measurement in order to clean the bore and tip of the syringe. The drops were observed and photographed on Polaroid film through a Iow power microscope and the photographs were analyzed on an $X-Y$ measuring microscope. The pendant drop technique requires the measurement of $\mathrm{d}_{e}$ the equatorial diameter and $\mathrm{d}_{s}$, the diameter measured at a height $\mathrm{d}_{\mathrm{e}}$ from the bottom of the drop. These dimensions are used to calculate a shape determining variable $\mathrm{S}=\mathrm{d}_{\mathrm{e}} / \mathrm{d}_{\mathrm{s}} \cdot$ Solutions to the Young-Laplace equation for the pendant drop characterize the drop profile in terms of a shape dependent quantity $\mathrm{H}$ which is a function of $\mathrm{S}$. Tables of $\mathrm{H}$ as a function of $\mathrm{S}$ permit the calculation of the surface tension from the relationship $\gamma=\frac{\rho \mathrm{gd}_{\mathrm{e}}{ }^{2}}{\mathrm{H}}$, where $\rho$ is the density and $g$ is the gravitational acceleration. The magnification of each picture is determined precisely by measurement of the diameter of the syringe tip at a 
specific cross section marked by a notch. A typical drop is shown in Figure 1.

\section{II . EXPER IMENTAL RESULTS}

We have found a time dependence of the gallium surface tension which is complex and qualitatively different from that observed by Abbaschian. The changes in surface tension with time we observe appear to be dependent on the helium flow rate. Figure 2 shows the surface tension as a function of time for a low flow rate, e.g., 4 bubbles/sec. The surface tension declines from about $710 \mathrm{~mJ} / \mathrm{m}^{2}$ to about $629 \mathrm{~mJ} / \mathrm{m}^{2}$ in 2 hours. This differs from Abbaschian's observations in several respects: the surface tension values here are about $100 \mathrm{~mJ} / \mathrm{m}^{2}$ lower than Abbaschian's and the decay to a constant value takes much longer than in his experiments.

A quite different result is obtained with a high helium flow rate, e.g., 2 liters/min. As seen in Figure 3, the surface tension declines initially but rises sharply after about fifty minutes to a value near $780 \mathrm{~mJ} / \mathrm{m}^{2}$. The rise is not monotonic; reanalysis of the pictures confirms the several local minima seen in the data. Note also the large decrease in surface tension after the titanium getter was turned off at 420 minutes. These two curves represent the extremes of the observed time dependences we have observed. Other data lie between the results shown here. 
Conclusions and Discussions

The complex time dependent surface tensions we observe are probably the result of several coupled dynamic processes. Although we do not understand the curves we think they reflect the diffusion of a bulk phase species to the surface with a subsequent desorption. Initially the flux of this species to the surface from the bulk is much faster than the desorption so the surface concentration increases and the surface tension falls. As this concentration builds up, however, the diffusion flux decreases and the desorption rate increases. If the bulk concentration of the impurity is decreased sufficiently, the diffusion flux will become smaller than the desorption flux and the surface concentration will decline leading to an increase in surface tension as seen in Figure 3. Eventually an equilibrium concentration is established on the surface which gives a constant surface tension. The gas phase impurities which come primarily from the cell walls and tubulation play a critical role in this equilibration as shown by the dependence on flow rate and on the use of the titanium getter.

The time dependent behavior described above postulates a bulk phase impurity which diffuses to the gallium surface and desorbs at room temperature. We can not identify the impurity at this point. It may be significant that the initial surface tensions of Abbaschian are much higher than those we observe. We believe this indicates his gallium is cleaner than ours. Although nominally six nines pure, our gallium comes in one gram ampoules whereas Abbaschian's gallium was in ingot 
form. The subdivision of the zone refined gallium for packaging in the ampoules may have introduced impurities. It is also possible the impurity is introduced from the alcohol and hydrochloric acid used in the handling procedure discussed earlier.

To resolve this complex picture it will probably be necessary to measure the gallium surface tension in ultra high vacuum. A system to do this is being assembled.

We note that two articles, based on previous work on this task, have been published during the past year, viz. "Stability of Liquid Zones", S. R. Coriell, S. C. Hardy, and M. R. Cordes, J. Colloid and Interface Science 60,126 (1977) and "Theory of Molten Zone Shape and Stability", S. R. Coriell and M. R. Cordes, J. Crystal Growth, 42, 466 (1977). An article "The Motion of Bubbles in a Vertical Temperature Gradient" by S. C. Hardy is in preparation. 


\section{References}

[1] T. W. Richards and S. Boyer, J. Amer. Chem. Soc. 43, 283 (1931).

[2] G. L. Mack, J. K. Davis and F. E. Barte11, J. Phys. Chem. 45, $846(1941)$.

[3] 0. A. Timofeevicheva and P. P. Pugachevich, Handbook of the Rare Elements. Vol. 1, Eds. M. A. Filyand and E. I. Semenova, M. E. Alferieff (Transl.) (MacDonald Technical and Scientific, London 1968) p. 13 .

[4] G. J. Abbaschian, Journal of Less-Common Metals 4C, 329 (1975). 


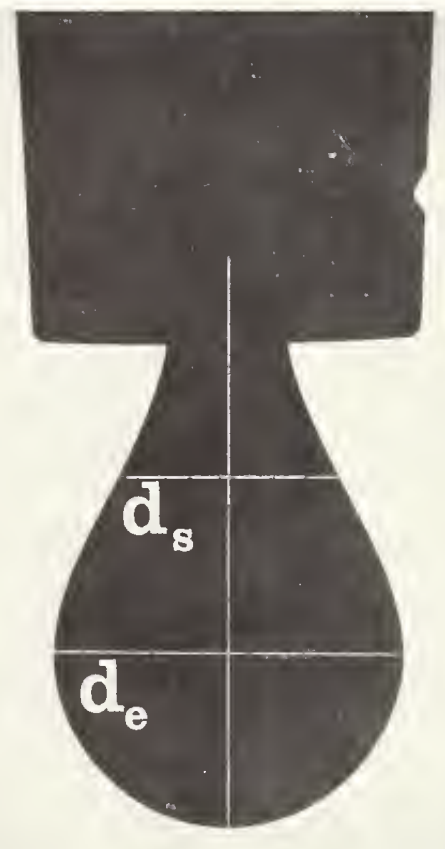

Fig. 1. Photograph of a pendant drop of gallium showing the dimensions which are used to calculate surface tension. For this drop the calculated surface tension was $790 \mathrm{~mJ} / \mathrm{m}^{2}$. 


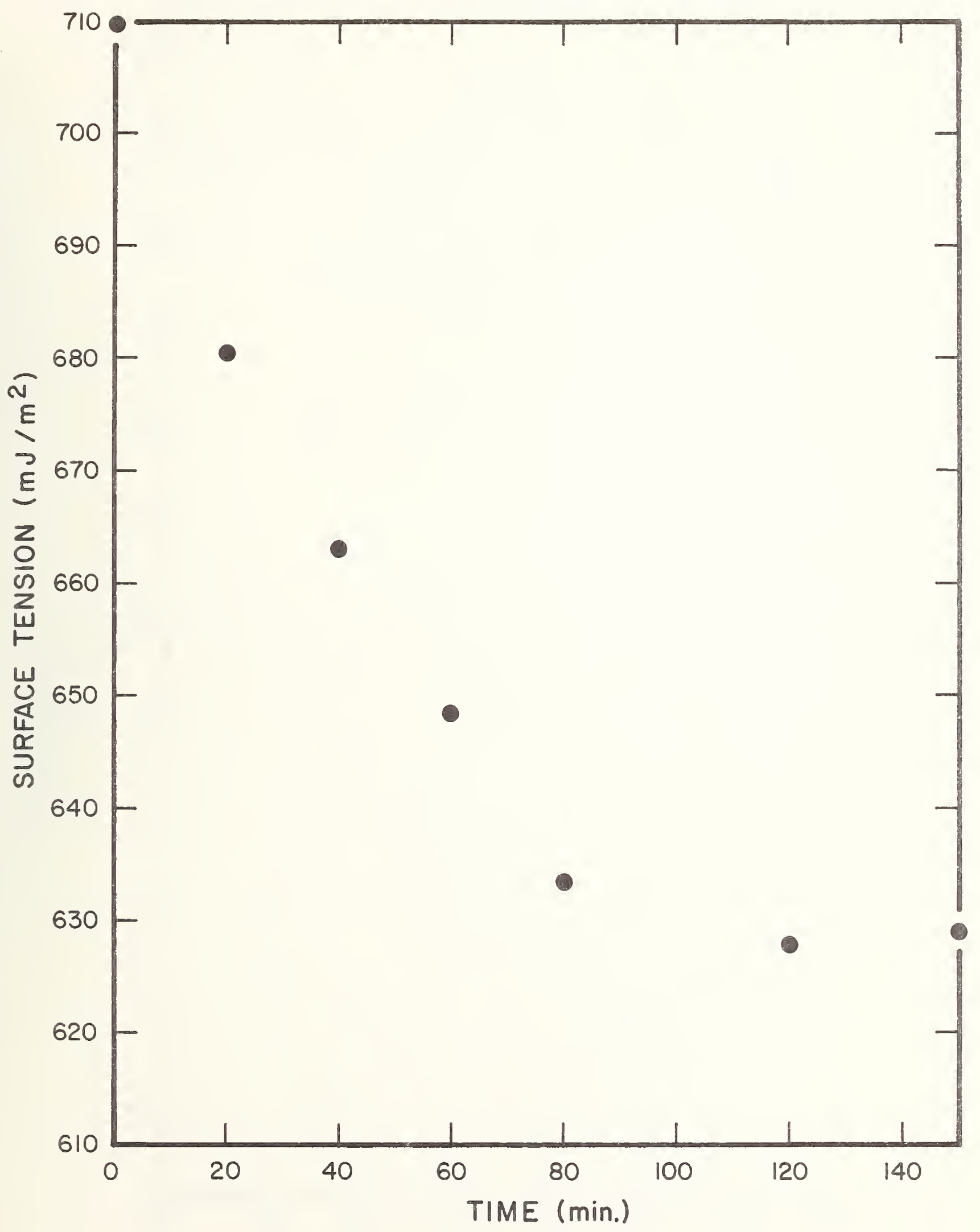

Fig. 2. The variation of surface tension with time for a low helium flow rate. 


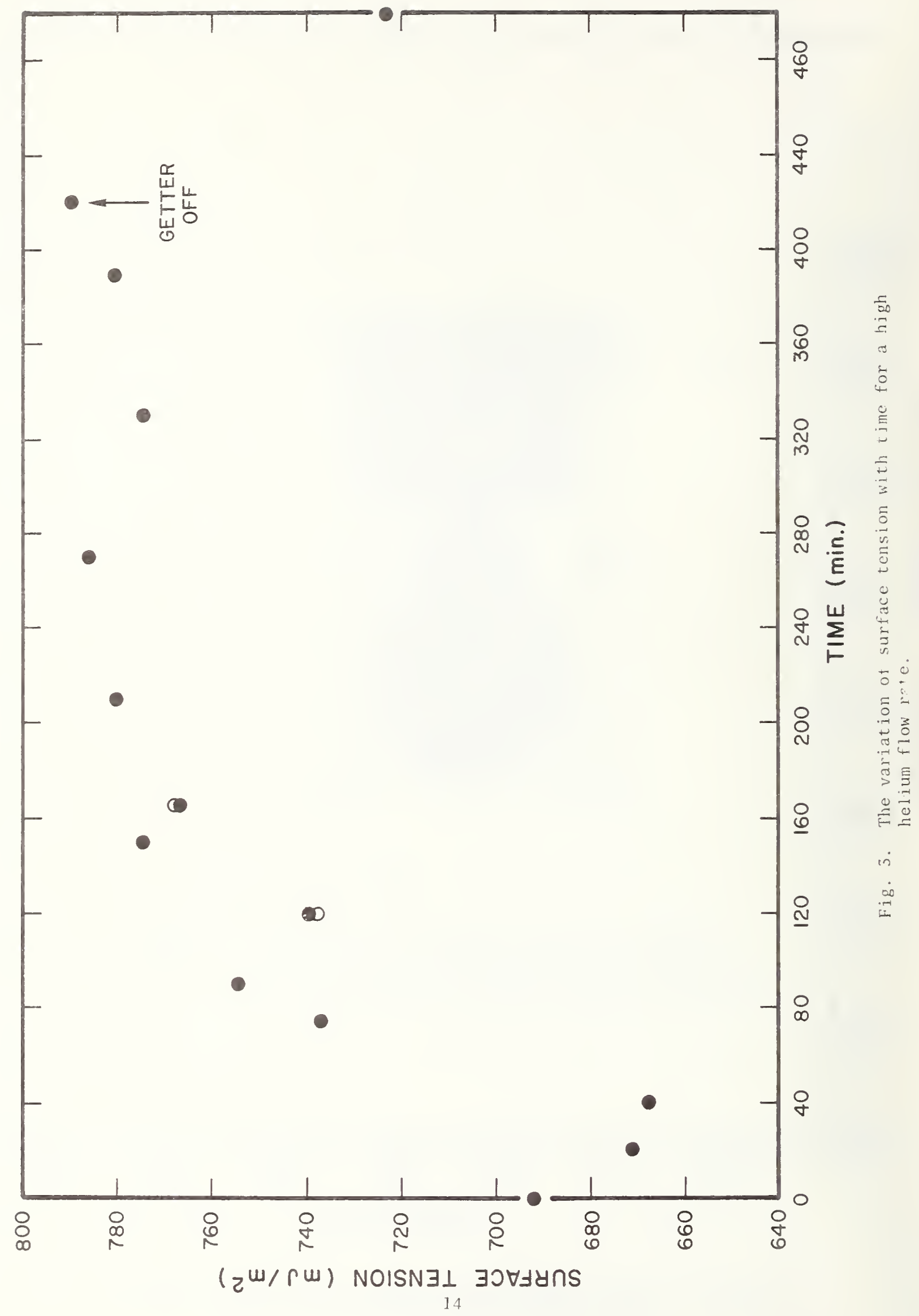


Task 2

Solutal Convection and Liquid Diffusion Coefficients

W. J. Boettinger, S. R. Coriell, and F. S. Biancaniello

Metal Science and Standards Division

Center for Materials Science

and

M. R. Cordes

Mathematical Analysis Division

Center for Applied Mathematics

Summary

This research is directed towards an understanding of solutal convection caused by the simultaneous concentration and temperature gradients present during the directional solidification of alloys. Included in this study is investigation of the effects of convection on the microstructure and chemical homogeneity of directionally solidified alloys and of the conditions under which undesirable effects can be eliminated.

The onset of convective and interfacial instabilities during the vertical directional solidification of a binary alloy is determined theoretically by means of a linear stability analysis. For values of the physical constants appropriate to the solidification of lead containing tin, the critical bulk solute concentration delineating the demarcation between stability and instability is numerically calculated as a function of growth velocity. For concentrations less than the critical concentration, the system is stable. For growth velocities 
between 1 and $40 \mathrm{\mu m} / \mathrm{s}$, the critical concentration increases with increasing velocity; for velocities greater than approximately $40 \mathrm{\mu m} / \mathrm{s}$, it decreases with velocity.

The occurrence of solutal convection and its effects on the microstructure and macrosegregation of directionally solidified alloys, particularly off-eutectics, is investigated using lead-tin alloys. Lead-rich lead-tin alloys have been directionally solidified vertically upwards. During plane front solidification the concentration of less dense solute is increased in the liquid near the solid-liquid interface and there is the possibility of solutal convection. In this year's research, procedures for careful directional solidification and sanple characterization have been developed and preliminary microstructural information on lead-rich lead-tin off-eutectics has been obtained. 


\section{INTRODUCTION}

In recent years there has been extensive research and development efforts on the effect of fluid flow on solidification and on the properties of the resulting solids $[1-2]$. In general, one attempts to control fluid flow in such a way as to obtain optimum materials properties. To minimize fluid flow, it is well known that horizontal temperature and concentration gradients must be avoided. If the fluid density gradient is parallel to the gravitational field, a motionless solution of the fluid flow equations is possible; in the remaining discussion we will assume that this is the case, i.e., there are no horizontal density gradients.

In the plane front constant velocity directional solidification of a11 alloys other than congruent melting compounds and invariant eutectics, there exists in the liquid an exponential concentration gradient which extends ahead of the liquid-solid interface with a decay distance of $\mathrm{D} / \mathrm{V}$ where $\mathrm{D}$ is the liquid interdiffusion coefficient and $\mathrm{V}$ is the interface velocity. There also exists a temperature gradient which is essentially linear in the region in which the concentration gradient is exponential. The density, being a function of temperature and concentration, can have various profiles depending on the solidification conditions and the properties of the fluid. The simplest criterion of whether convection occurs might be based on whether the density decreases or increases with height in the fluid [3]. However, this criterion will be shown to be incorrect. The complex interaction 
of the solute gradient and temperature gradient on the presence or absence of fluid flow and its effect on the freezing solid is the subject of this research. We will call convection caused by simultaneous concentration and temperature gradients, solutal convection. Solidification

Previous metallurgical work on solutal convection during solidification has mostly been directed towards off-eutectic and dendritic solidification. In 1967, Mollard and Flemings [4] showed that fully lamellar structures could be solidified vertically upward from compositions on the $\mathrm{Sn}-\mathrm{rich}$ side of the $\mathrm{Pb}-\mathrm{Sn}$ eutectic. In this regime the temperature gradient and the concentration gradient in the liquid are such that no convection is expected. Their paper claimed that on the $\mathrm{Pb}$-rich side, however, convection would be present because the density increases with height due to the concentration gradient and that growth of composites was impossible. However, Holder and Oliver [5] succeeded in growing composites on the $\mathrm{Pb}-\mathrm{rich}$ side up to 8 w/o from the eutectic and reported no macrosegregation. Hogan and Davis ${ }^{[6]}$ showed data for the dendrite to composite transition in Pb-rich $\mathrm{Sn}-\mathrm{Pb}$ alloys implying that no unusual problems were encountered. Verhoeven, Kingery, and Hofer ${ }^{[7]}$ in very careful experiments, reported extensive macrosegregation in Pb-rich alloys only $1 \mathrm{w} / \mathrm{O}$ from the Pb-Sn eutectic. Fitting their macrosegregation by a Burton-Prim-S1ichter ${ }^{[8]}$ type analysis modified for thermotransport ${ }^{[9]}$, they obtained $\delta$ parameters (width of unmixed zone at the liquid-solid 
interface) of as sma11 as $60 \mu \mathrm{m}$, which for these experiments indicates rather strong convection. Vandenbulcke and Vuillard ${ }^{[10]}$ solidified $\mathrm{Sn}-\mathrm{rich} \mathrm{Pb}-\mathrm{Sn}$ off-eutectics vertically downward. Here both temperature gradient and solute gradient contribute to a density which increases with height. (As will be pointed out in the next section, this may or may not cause convection.) Their work indicates macrosegregation is present. Thus, the effect that solutal convection can have on directional solidification is thought by these authors to fall into three areas: 1) it prevents the growth of the composite (or perhaps increases the fault density); 2) it causes macrosegregation in the composite; 3) it has no effect. It appears at this time that 2) is the most likely effect. However, the conditions under which solute convection occur and its effects on microstructure or macrosegregation are not fully known.

Macrosegregation in off-eutectics would be deleterious to the performance of these composites for practical purposes, e.g., turbine blades. It is important to know the extent of restrictions on composite growth because designers of these materials need flexibility in adjusting the volume fraction of the reinforcing phase and in adding alloying components to control high temperature microstructural stability and to improve strength and corrosion resistance. These alloying additions will make eutectics freeze over a range of temperature. These noninvariant eutectics in multicomponent systems behave just like off-eutectics in binary alloys as far as solutal convection is concerned. Hence the study of simple off-eutectics has wider 
applicability.

Other work hás been performed on solutal convection during dendritic and cellular solidification [11]. Macrosegregation down the length of ingots and certain casting defects (A-segregates and freckles) have been attributed to this flow. In these situations, horizontal solute and temperature gradients exist making this a much more complex problem than is presently being considered here.

\section{Convection}

A classic problem is that of the onset of convection when a pure liquid, whose density decreases with increasing temperature, is heated from below. For sufficiently small temperature gradients, there is no fluid flow. Convection occurs when the temperature gradient exceeds a certain critical value. Therefore convection can occur when more dense liquid is on top of less dense liquid. We shall refer to a non-increasing density with height as a statically stable density profile; if this is not the case we refer to it as statically unstable. For the case of a pure liquid heated from below, a statically unstable density profile does not necessarily cause convection; convection occurs when the density gradient exceeds a critical value. Extensive research has been carried out on the onset of convection [12-16].

More recently there has been research on the phenomena of solutal convection (also called double-diffusive, thermohaline or thermosolutal convection ${ }^{[13-27]}$. This phenomena occurs when the fluid density depends on two variables such as temperature and solute concentration, which have different diffusivities. An interesting aspect of solutal 
convection is the occurrence of convection even when the density profile is statically stabie. For this example, we assume that the solute is more dense than the solvent. Consider a fluid with a statically stable density profile which is heated from above (positive temperature gradient) and has a greater concentration of solute at the top (positive solute gradient). The positive temperature gradient alone would cause a negative density gradient and the positive solute gradient alone would cause a positive density gradient; we assume that the temperature gradient is sufficiently large that the net fluid density gradient is negative, i.e., the fluid is statically stable. Consider an element of fluid near the bottom of the container; it is cold and dilute in solute concentration. Imagine a disturbance in which this fluid element rises. Since the thermal diffusivity is greater than the solute diffusivity, the fluid element increases in temperature but does not change in solute concentration. At its new position it has nearly the same temperature but less solute than the surrounding fluid; it is thus less dense than the surrounding fluid and hence continues to rise, leading to convection. Thus convection occurs even though the density profile is statically stable. Clearly the static density profile is not useful in predicting convection, since one can have a statically unstable density profile without convection and a statically stable density profile with convection.

$\underline{\text { Research Plan }}$

In this task, the problem will be approached theoretically and experimentally. The question of when convection occurs during 
directional solidification is being investigated theoretically using a linear hydrodynamic stability analysis. This analysis of solutal convection is being performed for the solidification of a single phase material. Single phase materials are being investigated because of their intrinsic importance and because the problem is tractable. Results obtained here will be applicable to off-eutectics to describe the macroscopic features of their solidification. In the first year's work, the problem has been formulated, computer algorithms developed and initial results obtained for a limited number of cases.

The questions of whether solutal convection occurs and of what effects it has on the microstructure and possible macrosegregation of directionally solidified alloys, particularly off-eutectics, is being investigated experimentally using $\mathrm{Pb}-\mathrm{Sn}$ alloys. This alloy system is chosen because more data exists for this alloy system than others and because it is a low temperature alloy. Results will be generalized to other alloy systems. Pb-rich $\mathrm{Pb}-\mathrm{Sn}$ alloys have been directionally solidified vertically upwards where a less dense solute is built up in the liquid at the liquid- solid interface. Evidence for fluid flow is being sought. The presence of fluid flow can be detected by measuring variations in composition and/or microstructure along the growth direction in the solidified sample and changes in these observables when a magnetic field is applied. Such fields are known to increase the effective viscosity of liquid metals and retard convection. Also it will be determined whether a high temperature gradient can suppress 
solutal convection. In the first year's work, procedures for careful directional solidification and sample characterization have been developed and preliminary microstructural information on $\mathrm{Pb}-\mathrm{rich}, \mathrm{Pb}-\mathrm{Sn}$ off-eutectics have been obtained.

\section{THEORETICAL INVESTIGATION}

In studying the onset of convection, one uses a linear stability analysis. One considers an unperturbed state in which the temperature, concentration, density, pressure, and fluid flow velocities are functions of height alone, and satisfy the relevant differential equations. One then considers small disturbances in these quantities and retains only linear terms in the disturbances in the differential equations. In this linear theory, the time dependence of the disturbances is of the form $\exp (\sigma t)$, where $t$ is the time. Upon carrying out the calculation for a particular set of physical variables, e.g. temperature gradient, solute gradient, viscosity, etc., one determines $\sigma$. If the real part of $\sigma$ is positive, then the disturbances increase exponentially with time; the unperturbed state is unstable and convection occurs. If the real part of $\sigma$ is negative for all possible disturbances, the system is stable. The onset of convection corresponds to the set of physical variables for which the real part of $\sigma$ vanishes for some particular disturbance and is negative for all other disturbances. For example, we might fix all physical variables except the temperature gradient, and determine the critical value of the temperature gradient at the onset of convection. This critical temperature gradient is then the demarcation between stable (no convection) and unstable (convection) 
behavior. The onset of convection can occur in two different ways depending on whether or not the imaginary part of sigma vanishes when the real part of sigma vanishes. Writing $\sigma=\sigma_{r}+i \sigma_{i}$, if $\sigma_{i}=0$ when $\sigma_{r}=0$, the principle of the exchange of stabilities holds and the onset of convection corresponds to a monotonic (exponential) increase in the size of the disturbances. If $\sigma_{i} \neq 0$ when $\sigma_{r}=0$, there is overstability and the disturbances oscillate in time with exponentially increasing amplitude at the onset of convection.

Linear hydrodynamic stability theory for solutal convection with rigid and/or free boundary conditions and constant temperature and solute gradients has been developed by numerous scientists [13-22]. Some research has been carried out on non-linear theories of solutal convection [23-27]. However, the onset of convection during the vertical directional solidification of a binary alloy differs in several ways from previous treatments of solutal convection. Since the solid-liquid interface is moving at constant velocity $\mathrm{V}$, the concentration gradient is not constant, but decreases exponentially with distance $z$ from the interface, i.e. the concentration gradient decreases as $\exp (-V z / D)$, where $D$ is the diffusion coefficient in the liquid. The temperature gradient is also of this form with D replaced by $k$, the thermal diffusivity in the liquid. However, the quantity $(\mathrm{V} z / \mathrm{K})$ is usually sufficiently small that the thermal gradient is approximately constant. The boundary conditions at the solid-liquid interface are different from those used in treatments of double- 
diffusive convection. The solid-liquid interface can change shape as a result of fluctuations, and the solidification boundary conditions are applied at the actual solid-liquid interface.

In the absence of convection the stability of the shape of a solidliquid interface during solidification has been treated extensively in the last fifteen years by morphological stability theory [28-29]. During directional solidification a non-planar interface will give rise to a non-uniform solute distribution in the solidified material. A non-planar interface can occur without convection through the usual morphological instability mechanism. Clearly, convection can also cause a non-planar solid-liquid interface. Of course these are not distinct problems; both are governed by the same differential equations and boundary conditions. Previous morphological stability theory has assumed that the liquid density is independent of temperature and solute concentration. In this report we will generalize morphological stability theory and remove this assumption. We will also take account of the density change on solidification. Thus, one may look on the present calculations as double diffusive convection with solidification occurring or as morphological stability theory with fluid flow. They are simply different vantage points for the same physical problem.

We will present a few results for the case of directional solidification upwards of a binary alloy, which is rejecting solute which is less dense than the solvent, e.g., a small concentration of tin in lead. The temperature gradients are positive; i.e., the temperature 
increases with height. The temperature gradient contributes to a negative liquid density gradient, i.e., a statically stable density gradient. The concentration gradient contributes to a positive liquid density gradient. However, the concentration gradient exponentially decreases with distance from the solid-liquid interface with decay distance $\mathrm{V} / \mathrm{V}$; typically $\mathrm{D} / \mathrm{V}$ is of the order of 0.1-1.0 mm. Thus, near the solid-liquid interface the density gradient may be statically stable or unstabie depending on the relative magnitudes of the temperature and concentration gradients. Far from the interface the concentration gradient vanishes and the density gradient is statically stable. Our calculations will determine the conditions for the onset of convection and interface instability in such a system. 


\section{Theory}

We consider unidirectional vertical solidification of a binary alloy at constant velocity. We choose an $\left(x^{\prime}, y^{\prime}, z^{\prime}\right)$ 1aboratory coordinate system such that the $z^{\prime}$ axis is perpendicular to the planar solid-liquid interface. The gravitational field is of the form $\bar{g}=(0,0,-g)$, i.e., positive $g$ implies a field anti-parallel to the $z^{\prime}$ axis. The following differential equations govern the fluid velocity $\bar{u}=\left(u_{x^{-}}, u_{y^{\prime},}, u_{z^{-}}\right)$, temperature $T$, and concentration $\mathrm{c}^{[12-18]}$

$$
\begin{aligned}
& \operatorname{div} \bar{u}=0 \\
& \left(\partial \bar{u} / \partial t^{\prime}\right)+\left(\bar{u} \nabla^{\prime}\right) \bar{u}=-\left(\nabla p / \rho_{0}\right)+\nu \nabla^{\cdot 2} \bar{u}-\bar{g}\left[\alpha(T-\tilde{T})+\alpha_{c}\left(c-c^{2}\right)\right] \\
& \left(\partial T / \partial t^{\prime}\right)+\bar{u} \nabla^{\prime} T=\kappa \nabla^{\prime}{ }^{2} T, \\
& \left(\partial c / \partial t^{\prime}\right)+\bar{u} \nabla^{\prime} c=D \nabla^{\prime 2} c,
\end{aligned}
$$

where $t^{\prime}$ is the time, $p$ is a pressure, $v$ is the kinematic viscosity, $k$ is the liquid thermal diffusivity, and $\mathrm{D}$ is the liquid diffusion coefficient. The prime on the operator $\nabla$ indicates that it is with respect to the $\left(x^{\prime}, y^{\prime}, z^{\prime}\right)$ coordinate system. We have assumed that the fluid density $\rho$ is given by $\rho=\rho_{0}\left[1-\alpha(T-\tilde{T})-\alpha_{c}(c-\tilde{c})\right]$, where $\rho_{0}$ is the density at $T=\tilde{T}$ and $c=\tilde{c}$. The above equations are based on the Oberbeck-Boussinesq approximation ${ }^{[14-15]}$ and the coefficients $\nu, k$, and D are assumed constant.

We transform to a moving coordinate system $(x, y, z)$ attached to the unperturbed planar solid-liquid interface which is moving with constant velocity $\bar{V}=(0,0, V)$ in the $z$ - direction, i.e., $x=x^{\prime}, y=y^{\prime}, z=$ $z^{\prime}-V t$, and $t=t^{\prime}$. 
We obtain

$$
\begin{aligned}
& \operatorname{div} \bar{u}=0 \\
& (\partial \bar{u} / \partial t)-(\bar{V} \nabla) \bar{u}+(\bar{u} \nabla) \bar{u}=-\left(\nabla p / \rho_{0}\right)+v \nabla^{2} \bar{u}-\bar{g}\left[\alpha(T-\tilde{T})+\alpha_{c}(c-\tilde{c})\right](6) \\
& (\partial T / \partial t)-\bar{V} \nabla T+\bar{u} \nabla T=k \nabla^{2} T \\
& (\partial c / \partial t)-\bar{V} \nabla c+\bar{u} \nabla c=D \nabla^{2} c
\end{aligned}
$$

Note that $\bar{u}$ is still measured in the laboratory coordinate system $\left(x^{\prime}, y^{\prime}, z^{\prime}\right)$

The boundary conditions at the solid-liquid interface ${ }^{*}$ are that the tangential components of the fluid velocity vanish (no slip condition) and that the normal component satisfy

$$
\bar{v} \cdot \bar{n}\left(\rho_{L}-\rho_{S}\right)=(\bar{u} \cdot \bar{n}) \rho_{L},
$$

where $\bar{v}$ is the solidification velocity, $\bar{n}$ is the unit normal to the solid-liquid interface, and $\rho_{L}$ and $\rho_{S}$ are liquid and solid densities, respectively, at the solid-liquid interface. The temperature and concentration satisfy

$$
\begin{aligned}
& (\bar{v} \cdot \bar{n}) L_{v}=\left(-k_{L} \nabla T+k_{S} \nabla T S\right) \cdot \bar{n}, \\
& (\bar{v} \cdot \bar{n})\left(c_{S I}-c_{I}\right)=\left(\rho_{L} / \rho_{S}\right) D \nabla c \cdot \bar{n},
\end{aligned}
$$

where $L_{v}$ is the latent heat of fusion per unit volume of solid, $k_{L}$ and $k_{s}$ are thermal conductivities of 1 iquid and solid, respectively, $c_{s}$ and $c_{I}$ are solid and liquid concentrations at the solid-liquid interface, and all quantities are evaluated at the interface. The temperature $\mathrm{T}_{\mathrm{S}}$ in the solid satisfies the differential equation

$$
\left(\partial \mathrm{T}_{S} / \partial \mathrm{t}\right)-(\overline{\mathrm{V}} \nabla) \mathrm{T}_{\mathrm{S}}=\kappa_{\mathrm{S}} \nabla^{2} \mathrm{~T}_{\mathrm{S}},
$$

* The authors are indebted to R. F. Sekerka for detailed discussions and derivations of the boundary conditions at the solid-liquid interface. 
where $k_{s}$ is the thermal diffusivity of the solid. Diffusion in the solid has been neglected. In addition, we have

$$
\begin{aligned}
& \mathrm{T}_{S I}=\mathrm{T}_{\mathrm{I}} \\
& c_{s I}=k c_{I} \\
& (\bar{v} \cdot \bar{n})=\mu\left\{T_{e}-T_{I}\right\} \\
& \mathrm{T}_{\mathrm{e}}=\mathrm{T}_{\mathrm{N}}+\mathrm{HC}_{\mathrm{I}}-\mathrm{T}_{M} \Gamma \mathrm{K} \text {. }
\end{aligned}
$$

Here, we have used a subscript I to denote evaluation of the quantity at the solid-liquid interface; $k$ is the partition coefficient, $\mu$ is a linear Einetic coefficient, $\mathrm{T}_{e}$ is the equilibrium temperature of a curved interface in the presence of solute, $T_{M}$ is the melting point of the pure substance with a planar interface, $m$ is the slope of the liquidus line, $\Gamma$ is the capillary constant, i.e., the solid-liquid surface tension $\gamma=\mathrm{L}_{\mathrm{V}} \Gamma$, and $\mathrm{K}$ is the interface curvature.

We now solve the equations for a planar solid-liquid interface located at $z=0$; all quantities are functions of $z$ alone. We assume that in the moving frame there is no dependence on time. The fluid velocity, temperature, and concentration fields are

$$
\begin{aligned}
& \overline{\mathrm{u}}_{0}=(0,0,-\varepsilon V) \\
& \mathrm{T}_{0}=\mathrm{d}_{1}-\left(\mathrm{kG}_{\mathrm{L}} / \mathrm{V}^{*}\right) \exp \left(-\mathrm{V}^{*} \mathrm{z} / \mathrm{k}\right) \\
& \mathrm{T}_{\mathrm{s} 0}=\mathrm{d}_{2}-\left(\mathrm{k}_{\mathrm{S}} \mathrm{G}_{\mathrm{s}} / \mathrm{V}\right) \exp \left(-\mathrm{Vz} / \mathrm{k}_{\mathrm{s}}\right) \\
& \mathrm{c}_{0}=c_{\infty}-\left(c_{\infty} / \mathrm{k}\right)(\mathrm{k}-1) \exp \left(-\mathrm{V}^{*} \mathrm{z} / \mathrm{D}\right),
\end{aligned}
$$

where $\varepsilon=\left(\rho_{s} / \rho_{L}\right)-1, d_{1}$ and $d_{2}$ are constants, $V^{*}=V\left(\rho_{S} / \rho_{L}\right), G_{L}$ and $G_{S}$ are temperature gradients in the liquid and solid, respectively, at the solid-liquid interface, $c_{\infty}$ is the solute concentration far from the 
interface, and the subscript 0 indicates that the solutions are for an unperturbed planar interface. From the boundary conditions we also have the relationships

$$
\begin{aligned}
& V L_{v}=k_{s} G_{S}-k_{L} G_{L} \\
& V^{*}\left(c_{\infty} / k\right)(k-1)=D_{c} \\
& d_{1}-\left(k G_{L} / V^{*}\right)=d_{2}-\left(k_{s} G_{s} / V\right) \\
& c_{s I}=c_{\infty} \\
& V=\mu\left\{T_{M}+\left(m c_{\infty} / k\right)-d_{1}+\left(k G_{L} / V^{*}\right)\right\}
\end{aligned}
$$

where $G_{C}$ is the concentration gradient in the liquid at the solid-liquid interface. These relationships may be used to find explicit expressions for the constants $\mathrm{d}_{1}$ and $\mathrm{d}_{2}$.

We now consider whether the above solutions, for which there is only a trivial fluid flow corresponding to the density change on solidification, are stable with respect to small fluctuations. We write

$$
\begin{aligned}
& \bar{u}=\bar{u}_{0}+\bar{W}(x, y, z, t) \\
& T=T_{0}+T(x, y, z, t) \\
& T_{s}=T_{s 0}+T_{s}(x, y, z, t) \\
& c=c_{0}+C(x, y, z, t) \\
& p=p_{0}+p_{1}(x, y, z, t) \\
& z=z_{S L}(x, y, t)
\end{aligned}
$$

and will retain only linear terms in the perturbation quantities, $\bar{W}$, $T, T_{s}, C, p_{1}$, and $z_{s L}$. The function $z_{s L}(x, y, t)$ represents the shape of the solid-liquid interface which in general is not planar and the function $p_{0}(z)$ satisfies the unperturbed equations. We obtain the following differential equations for the fluctuations 


$$
\begin{aligned}
& \operatorname{div} \bar{W}=0 \\
& (\partial \bar{W} / \partial t)-V^{*}(\partial \bar{W} / \partial z)=-\left(\nabla p_{1} / \rho_{0}\right)+v \nabla^{2} \bar{W}-\bar{g}\left(\alpha T+\alpha_{C} C\right), \\
& (\partial T / \partial t)-V^{*}(\partial T / \partial z)+W_{z}\left(\partial T_{0} / \partial z\right)=k \nabla^{2} T, \\
& (\partial C / \partial t)-V^{*}(\partial C / \partial z)+W_{z}\left(\partial c_{0} / \partial z\right)=D \nabla^{2} C, \\
& \left(\partial T_{s} / \partial t\right)-V(\partial T / \partial z)=k_{S} \nabla^{2} T_{S} .
\end{aligned}
$$

Since equation (33) is a vector equation, we have seven equations for the seven functions $w_{x}, w_{y}, w_{z}, p_{1}, T, C$, and $T_{s}$. By appropriate rearrangement of these equations, we can eliminate $W_{x}, w_{y}$, and $p_{1}$, and obtain a higher order equation for $W_{z}$. Briefly, we apply the operator $\left(\partial^{2} / \partial z \partial x\right)$ to the $w_{x}$ equation, the operator $\left(\partial^{2} / \partial z \partial y\right)$ to the $w_{y}$ equation, and the operator $\nabla_{1}{ }^{2} \equiv\left(\partial^{2} / \partial x^{2}\right)+\left(\partial^{2} / \partial y^{2}\right)$ to the $w_{z}$ equation. Noting that from the continuity equation

$$
\left(\partial^{2} W_{x} / \partial x \partial z\right)+\left(\partial^{2} W_{y} / \partial y \partial z\right)+\left(\partial^{2} W_{z} / \partial z^{2}\right)=0
$$

we obtain after algebraic manipulation

$$
\left(\partial \nabla^{2} W_{z} / \partial t\right)-V^{*}\left(\partial \nabla^{2} W_{z} / \partial z\right)-\nu \nabla^{4} W_{z}=g \nabla_{1}^{2}\left(\alpha T+\alpha_{c} C\right),
$$

which is equivalent to equations (32) and (33).

The boundary conditions on the perturbed quantities at $z=0$ follow from the general boundary conditions and are

$$
\begin{aligned}
W_{z} & =-\varepsilon\left(\partial z_{S L} / \partial t\right) \\
W_{x} & =\varepsilon V\left(\partial z_{S L} / \partial x\right) \\
W_{y} & =\varepsilon V\left(\partial z_{s L} / \partial y\right) \\
\left(\partial z_{S L} / \partial t\right) L_{V} & =-k_{L}\left[-\left(V^{*} / K\right) G_{L} z_{S L}+\partial T / \partial z\right] \\
& \quad+k_{s}\left[-\left(V / k_{S}\right) G_{s} z_{S L}+\partial T_{s} / \partial z\right]
\end{aligned}
$$




$$
\begin{aligned}
& \left(\partial z_{S L} / \partial t\right)(1-k)\left(c_{\infty} / k\right)=k V G_{c} z_{S L}-(1-k) V C-\left(\rho_{L} / \rho_{S}\right) D(\partial C / \partial z) \\
& G_{S} z_{S L}+T_{S}=G_{L} z_{S L}+T \\
& \left(\partial z_{S L} / \partial t\right)=\mu\left\{m_{C} z_{S L}+m C+T_{M} \nabla_{1}{ }^{2} z_{S L}-G_{L} z_{S L}-T\right\}
\end{aligned}
$$

From the continuity equation, eqs (40) and (41) can be combined to give

$$
\left(\partial W_{z} / \partial z\right)=-\varepsilon V \nabla_{1} z_{S L}
$$

at $z=0$.

The equations and the boundary conditions are homogeneous and linear. It is convenient to represent the time dependence and the $\mathrm{x}$ and $y$ dependence in the form $\exp \left(\sigma t+i \omega_{x} x+i \omega_{y} y\right)$. The quantities $\omega_{x}$ and $\omega_{y}$ represent spatial frequencies and the general solution of the equations will involve integration over all values of $\omega_{x}$ and $\omega_{y}$. If the real part of $\sigma$ is positive for any values of $\left(\omega_{x}, \omega_{y}\right)$, then the solutions grow exponentially in time and are unstable. If the real part of $\sigma$ is negative for all values of $\left(\omega_{x}, \omega_{y}\right)$, the system is stable. The criterion for the onset of instability is determined by finding the conditions for which the real part of $\sigma$ is not positive for any $\left(\omega_{x}, \omega_{y}\right)$ and is equal to zero for some value of $\left(\omega_{x}, \omega_{y}\right)$. We write

$$
\begin{aligned}
& w_{z}=\hat{W}_{z}(z) \exp \left(\sigma t+i \omega_{x} x+i \omega_{y} y\right) \\
& T=\hat{T}(z) \exp \left(\sigma t+i \omega_{x} x+i \omega_{y} y\right) \\
& c=\hat{C}(z) \exp \left(\sigma t+i \omega_{x} x+i \omega_{y} y\right) \\
& T_{s}=\hat{T}_{s}(z) \exp \left(\sigma t+i \omega_{x} x+i \omega_{y} y\right) \\
& z_{S L}=\hat{z} \exp \left(\sigma t+i \omega_{x} x+i \omega_{y} y\right),
\end{aligned}
$$


where $\hat{W}_{z}, \hat{T}, \hat{C}, \hat{T}_{s}$ are functions of $z$ alone, and $\hat{z}$ is a constant. Substituting these expressions in the differential equations yields

$$
\begin{aligned}
& \left\{\left(\partial^{2} / \partial z^{2}\right)-\omega^{2}\right\}\left\{v\left[\left(\partial^{2} / \partial z^{2}\right)-\omega^{2}\right]+V^{*}(\partial / \partial z)-\sigma\right\} \hat{W}{ }_{z}=g \omega^{2}(\alpha \hat{T}+\alpha \hat{C}), \\
& \left\{k\left[\left(\partial^{2} / \partial z^{2}\right)-\omega^{2}\right]+V^{*}(\partial / \partial z)-\sigma\right\} \hat{T}=\hat{W}_{z} G_{L} \exp \left(-V^{*} z / k\right), \\
& \left\{D\left[\left(\partial^{2} / \partial z^{2}\right)-\omega^{2}\right]+V^{*}(\partial / \partial z)-\sigma\right\} \hat{C}=\hat{W}_{z} G_{C} \exp \left(-V^{*} z / D\right), \\
& \left\{\kappa_{S}\left[\left(\partial^{2} / \partial z^{2}\right)-\omega^{2}\right]+V(\partial / \partial z)-\sigma\right\} \hat{T}_{S}=0,
\end{aligned}
$$

where $\omega^{2}=\omega_{x}^{2}+\omega_{y}^{2}$.

Since the equation for $\hat{T}_{S}$ does not depend on $\hat{W}_{z}, \hat{T}$, or $\hat{C}$, it can be solved analytically. Using the boundary condition $\hat{T}_{S}(z \rightarrow-\infty=0$, we find

$$
\hat{T}_{\mathrm{S}}=\mathrm{d}_{3} \exp (\lambda z) \text {, }
$$

where $d_{3}$ is a constant and

$$
\lambda=\left(-V / 2 k_{s}\right)+\left\{\left(V / 2 k_{s}\right)^{2}+\omega^{2}+\left(\sigma / k_{s}\right)\right\}^{\frac{2}{2}} \text {. }
$$

We have three coupled differential equations for the functions $\hat{W}_{Z}$, $\hat{T}$, and $\hat{C}$. The boundary conditions, eqs $(39-46)$, for $\hat{W}_{z}, \hat{T}$, and $\hat{C}$ at $z=0$ also contain the unknown constant $\hat{z}$ and $\mathrm{d}_{3}$. However, we can eliminate these constants, and obtain boundary conditions for $\hat{W}_{z}, \hat{T}$, and $\hat{C}$ at $z=0$, viz,

$$
\begin{aligned}
& \hat{W}_{z}+\left(\sigma / V \omega^{2}\right)\left(\partial \hat{W}_{z} / \partial z\right)=0, \\
& \left(\partial \hat{W}_{z} / \partial z\right)+\left(\varepsilon V \omega^{2} k_{L} / a_{3}\right)(\partial \hat{T} / \partial z)-\left(\varepsilon V \omega^{2} k_{s} \lambda / a_{3}\right) \hat{T}=0, \\
& \left(a_{3}+a_{2} k_{s} \lambda\right) \hat{T}-\left(a_{2} k_{L}\right)(\partial \hat{T} / \partial z)-a_{3} m \hat{C}=0, \\
& -a_{4} \hat{T}+\left[m a_{4}+(1-k) V a_{2}\right] \hat{C}+\left(a_{2} D \rho_{L} / \rho_{s}\right)(\partial \hat{C} / \partial z)=0,
\end{aligned}
$$

where

$$
a_{1}=\left(k_{L} G_{L} V^{*} / K\right)-\left(k_{S} G_{S} V / k_{S}\right)
$$




$$
\begin{aligned}
& a_{2}=(\sigma / \mu)-m G_{c}+G_{L}+T_{M} \Gamma \omega^{2} \\
& a_{3}=\sigma L_{v}-a_{1}-k_{s} \lambda\left(G_{L}-G_{s}\right) \\
& a_{4}=\sigma(1-k)\left(c_{\infty} / k\right)-k V G_{c} .
\end{aligned}
$$

Thus we have four linear homogeneous boundary conditions at $z=0$. We need four additional conditions in the liquid away from the interface since our differential equations are equivalent to a single eighth order equation. We consider a rigid planar isothermal boundary at $z=z_{L}$ (a constant). The boundary conditions are

$$
\hat{W}_{z}=\left(\partial \hat{W}_{z} / \partial z\right)=\hat{T}=(\partial \hat{C} / \partial z)=0
$$

However, in order to be able to treat a more general class of problems, we will write the boundary conditions as $\hat{W}_{z}=0$,

$$
\begin{aligned}
& b_{1} \hat{T}+b_{2}(\partial \hat{T} / \partial z)=0, \\
& b_{3} \hat{C}+b_{4}(\partial \hat{C} / \partial z)=0, \\
& b_{5}\left(\partial \hat{W}_{z} / \partial z\right)+b_{6}\left(\partial \hat{W}_{z} / \partial z^{2}\right)=0
\end{aligned}
$$

where $b_{1}, b_{2}, b_{3}, b_{4}, b_{5}$, and $b_{6}$ are constants. Clearly $b_{1}=b_{4}=$ $b_{5}=1$ and $b_{2}=b_{3}=b_{6}=0$ corresponds to eq (66).

The boundary conditions at $z=z_{L}$ are not rigorous. In many solidification experiments, there is a rigid boundary at $z^{-}=z_{L}$ (a constant), which implies that in our moving frame the boundary conditions should be applied at $z=z_{L}-V t$. In addition, if $\rho_{L} \neq \rho_{s}$, the unperturbed flow is not zero at $z_{L}$. To take account of these effects would greatly complicate the calculation. We are basically assuming that as long as ${ }_{L}$ is sufficiently far from the solid-liquid interface, the results will be relatively independent of the precise boundary conditions. This assumption will be investigated by numerical calculations. 


\section{Numerical Methods}

It is convenient to convert the differential equations, eqs, (52-55) to eight first order equations. We define

$$
\begin{aligned}
Y_{1} & =\hat{W}_{z} \\
Y_{2} & =\left(\partial \hat{W}_{z} / \partial z\right) \\
Y_{3} & =\left(\partial \hat{W}_{z} / \partial z^{2}\right) \\
Y_{4} & =\left(\partial \hat{W}_{z} / \partial z\right) \\
Y_{5} & =\hat{T} \\
Y_{6} & =(\partial \hat{T} / \partial z) \\
Y_{7} & =\hat{C} \\
Y_{8} & =(\partial \hat{C} / \partial z)
\end{aligned}
$$

and denote by $\bar{Y}(z)$ the vector with components $Y_{i}(z)(i=1,2, \ldots 8)$. The differential equations are now

$$
\begin{aligned}
\left(\partial Y_{1} / \partial z\right) & =Y_{2} \\
\left(\partial Y_{2} / \partial z\right) & =Y_{3} \\
\left(\partial Y_{3} / \partial z\right) & =Y_{4} \\
\left(\partial Y_{4} / \partial z\right) & =-\left(V^{*} / \nu\right) Y_{4}+\left[2 \omega^{2}+(\sigma / \nu)\right] Y_{3}+\left(\omega^{2} V^{*} / \nu\right) Y_{2} \\
- & {\left[\omega^{2}+(\sigma / \nu)\right] \omega^{2} Y_{1}+\left[g \omega^{2} / \nu\right]\left[\alpha Y_{5}+\alpha_{c} Y_{7}\right] } \\
\left(\partial Y_{5} / \partial z\right) & =Y_{6} \\
\left(\partial Y_{6} / \partial z\right) & =-(V * / K) Y_{6}+\left[\omega^{2}+(\sigma / K)\right] Y_{5}+\left(Y_{1} G_{L} / K\right) \exp \left(-V^{*} z / K\right) \\
\left(\partial Y_{7} / \partial z\right) & =Y_{8} \\
\left(\partial Y_{8} / \partial z\right) & =-\left(V^{*} / D\right) Y_{8}+\left[\omega^{2}+(\sigma / D)\right] Y_{7}+\left(Y_{1} G_{c} / D\right) \exp \left(-V^{*} z / D\right)
\end{aligned}
$$

or in a matrix form

$$
(\partial \bar{Y} / \partial z)=A \bar{Y}
$$


where the elements of the $8 \times 8$ matrix A are defined by eqs (78-85). In general the functions $Y_{i}$ and the quantity $\sigma$ are complex. A further decomposition of the form $Y_{i}=U_{2 i-1}+i U_{2 i}$ and $\sigma=\sigma_{r}+i \sigma_{i}$ allows one to consider only real functions and quantities. This results in sixteen first order differential equations for the functions $U_{i}$ or in matrix form

$$
(\partial \bar{U} / \partial z)=A-\bar{U}
$$

where $\bar{U}$ is the vector with components $U_{i}$ and $A^{\prime}$ is a $16 \times 16$ real matrix whose components can be determined from eqs (78-85).

Since the equations for $Y_{i}(i=1,2, \ldots 8)$ are linear and homogeneous, there are eight linearly independent solutions. We will determine these eight solutions numerically $\mathrm{y}^{[30-33]}$. We define the $\mathrm{k}$ th linearly independent solution $\vec{Y}^{k}$ as a solution of the differential equation (86) satisfying the initial condition (i.e., at $z=0$ ) $Y_{i}{ }^{k}(0)=\delta_{i k}$ for $i=1,2, \ldots 8$, where $\delta_{i k}=0$ if $i \neq k$ and 1 if $i=k$. The $\bar{Y}^{k}$ are solutions of an initial value problem, and the numerical computer codes developed by Shampine and Gordon [34] have been used to calculate these functions. The general solution of the differential equations is then

$$
\bar{Y}=\sum_{k=1}^{8} p_{k} \bar{Y}^{k}
$$

where $p_{k}$ are constants.

The boundary conditions, eqs $(58-61)$ at $z=0$ and eqs (66) at $z=z_{L}$ can be written in the form

$$
B_{0} \bar{Y}(0)+B_{L} \bar{Y}\left(z_{L}\right)=0
$$


where $\mathrm{B}_{0}$ and $\mathrm{B}_{\mathrm{L}}$ are $8 \times 8$ matrices whose elements are independent of $\mathrm{z}$ and are determined from the boundary conditions. For example, we can order the boundary conditions in such a way that the elements in the last four rows of $\mathrm{B}_{0}$ and the first four rows of $\mathrm{B}_{\mathrm{L}}$ vanish. Substituting eq (88) into eq (89) we obtain the vector equation,

$$
\sum_{k=1}^{8} p_{k}\left[B_{0} \bar{Y}^{k}(0)+B_{L} \bar{Y}^{k}\left(z_{L}\right)=0\right. \text {, }
$$

or in component form

$$
\sum_{k=1}^{8} p_{k} \sum_{j=1}^{8}\left[\left(B_{0}\right)_{i j} Y_{j}{ }^{k}(0)+\left(B_{L}\right)_{i j} Y_{j}{ }^{k}\left(z_{L}\right)=0, i=1,2, \ldots 8\right.
$$

where $\left(B_{0}\right)_{i j}$ and $\left(B_{L}\right)_{i j}$ are the elements in the $i$ th row and $j$ th column of the matrices $\mathrm{B}_{0}$ and $\mathrm{B}_{\mathrm{L}}$. Thus we have eight linear homogeneous equations for the eight coefficients $p_{k}$. A trivial solution of these equations is $p_{k}=0$ for all $k$. This is the only solution unless the determinant of the coefficients vanish, i.e.

$$
\operatorname{det} \mathrm{F}=0 \text {, }
$$

where the elements $\mathrm{F}_{i k}$ of the matrix $\mathrm{F}$ are given by

$$
F_{i k}=\sum_{j=1}^{8}\left[\left(B_{0}\right)_{i j} Y_{j}^{k}(0)+\left(B_{L}\right)_{i j} Y_{j}^{k}\left(z_{L}\right)\right] \text {. }
$$

Since $Y_{j}{ }^{k}(0)=\delta_{j k}$, we can also write

$$
F_{i k}=\left(B_{0}\right)_{i k}+\sum_{j=1}^{8}\left(B_{L}\right)_{i j} Y_{j}^{k}\left(z_{L}\right) \text {. }
$$

Explicit expressions for $\left(B_{0}\right)_{i k}$ and $\left(B_{L}\right)_{i k}$ are

$$
\begin{aligned}
& \left(\mathrm{B}_{0}\right)_{11}=1 \\
& \left(\mathrm{~B}_{0}\right)_{12}=\left(\sigma / \mathrm{V} \omega^{2}\right)
\end{aligned}
$$




$$
\begin{aligned}
& \left(B_{0}\right)_{22}=1 \\
& \left(B_{0}\right)_{25}=-\left(\varepsilon V \omega{ }^{2} k_{s} \lambda / a_{3}\right) \\
& \left(B_{0}\right)_{26}=\left(\varepsilon V \omega^{2} k_{L} / a_{3}\right) \\
& \left(B_{0}\right)_{35}=\left(a_{3}+a_{2} k_{s} \lambda\right) \\
& \left(B_{0}\right)_{36}=-\left(a_{2} k_{L}\right) \\
& \left(B_{0}\right)_{37}=-\left(a_{3} m_{1}\right. \\
& \left(B_{0}\right)_{45}=-a_{4} \\
& \left(B_{0}\right)_{47}=m_{4}+(1-k) V a_{2} \\
& \left(B_{0}\right)_{48}=a_{2} D_{L} / p_{s} \\
& \left(B_{L}\right)_{51}=1 \\
& \left(B_{L}\right)_{62}=1 \\
& \left(B_{L}\right)_{75}=b_{1} \\
& \left(B_{L}\right)_{76}=b_{2} \\
& \left(B_{L}\right)_{87}=b_{3} \\
& \left(B_{L}\right)_{88}=b_{4}
\end{aligned}
$$

with all other elements equal to zero.

Since $F$ is in general a complex matrix, eq (92) is equivalent to two equations, i.e., both the real and imaginary parts of det F vanish. For particular values of the parameters occuring in the differential equations and boundary conditions, det $F$ in general will not vanish. We will vary two parameters, viz., $\sigma_{i}$ and $c_{\infty}$, until $\operatorname{det} F$ vanishes. We use an iteration method developed by Brown [35] as implemented by Gay [36] to find values of $c_{\infty}$ and $\sigma_{i}$ for which det $F$ vanishes. 
Numerical Results

The differential equations and boundary conditions obtained in the previous sections depend on a large number of parameters. Even if one uses dimensionless variables, there appear to be thirteen dimensionless parameters necessary to specify the particular solidification conditions. A possible set of dimensionless parameters are $r=v / k$ (Prandt1 number), $s=v / D$ (Schmidt number), $R=-g a G_{L} z_{L}^{4} / \nu k$ (Rayleigh number), $S=-g \alpha_{c} G_{c} z_{L}^{4} / \nu D$ (solute Rayleigh number), $z_{L} V / \nu, \varepsilon, k_{S} / k, k$, $k_{s} / k_{L}, V L_{v} / k_{L} G_{L}, m_{c} / G_{L}, T_{M} \Gamma V^{2} / \nu^{2} G_{L}, V^{2} / \nu \mu G_{L}$. The first four $r, s, R$, and $\mathrm{S}$ are the usual dimensionless parameters for thermosolutal convection while the remaining parameters are related to the solidification process. Clearly, it is impossible to explore the effects of more than a few combinations of these variables on the onset of instability. In addition, the numerical code for performing the calculations was only recently completed so that a very limited set of results are available at present. Additional calculations will be carried out in the future. In conjunction with the experimental program, the first calculations have used parameters appropriate to the solidification of lead containing small quantities of tin. The specific values are given in Table 1 . We have initially taken the density of solid and liquid equal $(\varepsilon=0)$. In the usual morphological stability theory the liquid density is assumed (1) independent of temperature $(\alpha=0)$ and concentration $\left(\alpha_{c}=0\right)$ and (2) the density change on solidification is neglected $(\varepsilon=0)$. In order to distinguish 
between these two effects, our initial calculations have taken $\varepsilon=0$ and $\alpha$ and $\alpha_{c}$ non-zero. We have also neglected any kinetic effects, i.e., $\mu \rightarrow \infty$.

A number of tests of the numerical program have been carried out. The program was written so that the usual type of boundary conditions can be used at both $z=0$ and $z=z_{L}$, i.e., of the type given by eqs (67-69). A comparison of our numerical calculations with previous analytical and numerical results for thermal and thermosolutal convection is given in Table 2, and good agreement is found. Such calculations correspond to $V=0$ but $G_{c}$ and $G_{L}$ finite (see eqs $(52-54))$. When $\alpha=\alpha_{c}=\varepsilon=0$, there is no fluid flow and the equations can be solved analytically. For solidification boundary conditions, these are the usual morphological stability results (with boundary conditions at $z=z_{L}$ ). For this special case, our numerical calculations are in agreement with the analytic results.

The calculations carried out correspond to unidirectional solidification upwards of a binary alloy which is rejecting $(k<1)$ a solute whose density is less than that of the solvent $\left(\alpha_{c}>0\right)$. The density change due to solute is such that heavier fluid is above less dense fluid, i.e., the solute gradient promotes convection. The thermal gradient is positive so that the density change due to temperature is such that less dense fluid is above heavier fluid, i.e., the thermal gradient is stabilizing with respect to convection. The solute gradient falls off exponentially with a decay distance of $D / V^{*}$, whereas the 
temperature gradient is essentially linear since $k / V^{*}$ is typically much larger than $\mathrm{z}_{\mathrm{L}}$ (see eqs $\left.(18-20)\right)$. We expect therefore that for sufficiently small solute concentration there will be no convection, and the calculation will determine the solute concentration at which convection first occurs.

In carrying out a particular calculation, in addition to parameters specified in Table 1 , one specifies $V, z_{L}$, and $\omega$, takes $\sigma_{r}=0$, and picks initial values of $\sigma_{i}$ and $c_{\infty}$. The program then varies $\sigma_{i}$ and $c_{\infty}$ systematically until det $\mathrm{F}=0$; more precisely until the real and imaginary parts of $\operatorname{det} F$ are less than some prescribed value or until the changes in $\sigma_{i}$ and $c_{\infty}$ from one iteration to the next iteration are less than some prescribed value. We have also written a program which assumes that $\sigma_{i}=0$ when $\sigma_{r}=0$ (principle of the exchange of stabilities). In this case $F$ is real and $c_{\infty}$ is varied until det $F=0$. If the initial guess for $\sigma_{i}$ and $c_{\infty}$ (or $c_{\infty}$ alone) is not sufficiently close to the actual root of $\operatorname{det} F=0$, the iteration may not converge. In addition, there are many roots of $\operatorname{det} F=0$, and although we are primarily interested in the smallest value of $c_{\infty}$ (which we denote by $\mathrm{c}_{\infty}^{*}$ ), the iteration may converge to some larger root. For a particular

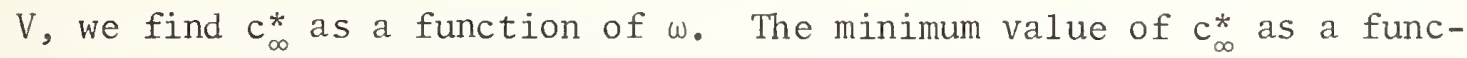
tion of $\omega$ is denoted by $c_{\infty}^{* *}$. Calculations allowing both $\sigma_{i}=0$ and $c_{\infty}$ to vary appeared to indicate that $\sigma_{i}=0$; therefore most calculations were carried out assuming $\sigma_{i}=0$ since these require considerably less computer time. However, as will be discussed, this assumption requires further investigation. 
The values of $z_{L}$ were chosen such that $z_{L}>>D / V^{*}$ and $\omega z_{L}>>1$. For $z_{L}$ satisfying these conditions the results for $c_{\infty}^{*}$ were independent of $z_{L}$; the results were also independent of the precise boundary conditions imposed at $z=z_{L}, e . g ., \hat{T}=\hat{C}=0$ boundary conditions gave the same result as $(\partial \hat{T} / \partial z)=(\partial \hat{C} / \partial z)=0$. As $\omega z_{L}$ becomes large, the present computer program fails. This occurs because the functions $\mathrm{Y}_{\mathrm{i}}{ }^{\mathrm{k}}$ become very large, viz., roughly like $\exp \left(\omega z_{L}\right)$. This results in long computation times and decreasing accuracy. Hence, we choose $z_{L}$ such that $1<<\omega z_{L}<20$ and $z_{L}>D / V^{*}$. As can be seen from Table 2, accurate results are obtained for $\omega z_{L}=20$. The program fails in a similar manner if $\sigma_{i}$ is not small, i.e., $\left(\sigma_{i} / D\right)^{\frac{1}{2}} z_{L}>1$. This currently precludes in.vestigating the existence of solutions corresponding to overstability $\left(\sigma_{i} \neq 0\right)$. In the corresponding problem with linear temperature and concentration gradients and free boundaries $[13,21]$ it is known that overstability does not occur when the temperature gradient is stabilizing and the concentration gradient destabilizing as is the case in the present solidification problem; overstability does occur $[13,21]$ when the temperature gradient is destabilizing and the solute gradient stabilizing. We plan to modify out numerical algoritlım so that results can be obtained for large $\omega z_{L}$ and $\sigma_{i}$.

The results of our numerical calculations are presented in Table 3 . For $c_{\infty}>c_{\infty}^{* *}$, convection will occur. As the growth velocity $V$ increases from $1.0 \mu \mathrm{m} / \mathrm{s}$ to $40 \mu \mathrm{m} / \mathrm{s}, c_{\infty}^{* *}$ increases and the wavelength $\lambda=2 \pi / \omega$ decreases. Clearly this is not a universal behavior, e.g., if $V \pm 0$, 
convection would not occur and $\mathrm{c}_{\infty}^{* *}$ is infinite. The results of Table 3 can be rationalized if one assumes that the relevant length scale for this particular problem is $\mathrm{D} / \mathrm{V}$. The independence of the results on the precise value of $z_{L}$ indicates that this is reasonable. We therefore define Kayleigh numbers based on (D/V), viz.

$$
\begin{aligned}
& R^{\prime}=-g \alpha G_{L}(D / V)^{4} / \nu K \\
& S^{\prime}=-g \alpha_{c} G_{C}(D / V)^{4} / \nu D=\left(g \alpha_{c} / \nu D k\right)(1-k) c_{\infty}(D / V)^{3} .
\end{aligned}
$$

For the values in Table $3,\left|R^{-}\right|<\left|S^{-}\right|$and for $V \geq 2.5 \mu \mathrm{m} / \mathrm{s}^{\prime}\left|R^{-}\right|<<\left|\mathrm{S}^{-}\right|$, By assuming that the onset of convection occurs when $S^{\prime}$ exceeds a critical value, one predicts that $\mathrm{c}_{\infty}^{* *}$ should increase with $V$. However, although the critical values of $\mathrm{S}^{\prime}$ given in Table 3 do not vary as much as $\mathrm{c}_{\infty}^{* *}$, they are certainly not constant. The liquid density decreases with height as long as $\alpha G_{L}+\alpha_{c} G_{c}>0$ or $c_{\infty}<c_{\infty}^{\prime \prime} \equiv\left(\alpha G / \alpha_{c}\right)[k /(1-k)](D / V)=$ $1.896(\mathrm{D} / \mathrm{V})^{+}$; this value is given in the last column of Table 3 . Only for the two largest velocities, viz., $V=40$ and $80 \mu \mathrm{m} / \mathrm{s}$ does the density increase with height at the onset of convection. For the other velocities, convection occurs even though the liquid density decreases with height.

For velocities much larger than $40 \mathrm{\mu m} / \mathrm{s}$, the solid-liquid interface is morphologically unstable for $c_{\infty}>0.215 \mathrm{w} / \mathrm{o}$. Neglecting surface tension, the morphological stability criterion for interface stability is $c_{\infty}<\left[\operatorname{Dk}\left(2 \mathrm{k}_{\mathrm{L}} \mathrm{G}_{\mathrm{L}}+\mathrm{VL} \mathrm{L}_{\mathrm{V}}\right)\right] /\left[\mathrm{mV}(\mathrm{k}-1)\left(\mathrm{k}_{\mathrm{L}}+\mathrm{k}_{\mathrm{S}}\right)\right] \simeq 25.7(\mathrm{D} / \mathrm{V})^{+}$. For morphological instability $\hat{C}$ and $\hat{T}$ are non-zero; it follows from eq (52) that $\hat{W}_{z}$ in

\footnotetext{
Here $D / V$ has units of $\mathrm{cm}$.
} 
general does not vanish. Thus, morphological instability implies convective instability. Similarly if $\hat{W}_{z}$ is non-zero, eqs $(53-54)$ imply that in general $\hat{T}$ and $\hat{C}$ are non-zero and convective instability inplies morphological instability. Since in the morphological stability criterion $c_{\infty}^{* *}$ is proportional to $\mathrm{V}^{-1}$, for velocities somewhat greater than $40 \mu \mathrm{m} / \mathrm{s}, c_{\infty}^{* *}$ will decrease with increasing $V$. The result in Table 3 for $V=80 \mu \mathrm{m} / \mathrm{s}$ illustrates the beginning of this trend; the value of $c_{\infty}^{* *}$ is in excellent agreement with previous morphological stability theory which neglects convection. This may be related to the quite different spatial frequencies at which morphological and convective instabilities occur; the convective instabilities occur at large wavelenths compared to the morphological instabilities. For the parameters chosen for our numerical calculations, it appears that it is impossible to avoid either convective or morphological instability, if the concentration is greater than $0.2 \mathrm{w} / \mathrm{o}$. In Figure 1 for $V=20 \mathrm{~m} / \mathrm{s}$, we illustrate the dependence of $c_{\infty}^{*}$ on $\omega$. The value of $c_{\infty}^{* *}$ corresponds to the minimum value of $c_{\infty}^{*}$, viz. $0.0189 \mathrm{w} / 0$ at $\omega=24$. When morphological and convective instabilities occur at about the same values of $c_{\infty}$, e.g. for $V=40 \mu \mathrm{m} / \mathrm{s}$, the corresponding plot of $c_{\infty}$ as a function of $\omega$ is more complicated and is still being investigated. For $\mathrm{V}=20 \mu \mathrm{m} / \mathrm{s}$, we have also varied the temperature gradient. For $G_{L}=100,200$, and $400 \mathrm{~K} / \mathrm{cm}$, the values of $\mathrm{c}_{\infty}^{* *}$ are $0.186,0.189$, and $0.195 \mathrm{w} / \mathrm{o}$ with $\omega=23,24$, and $25 \mathrm{~cm}^{-1}$, respectively. For this velocity and this range of temperature gradients, increasing $G_{L}$ has only a very small stabilizing effect. 
At present the number of calculations is so limited that general conclusions can not be drawn. There are a large number of areas that require further investigation. Calculations for a range of Prandt numbers and systems for which the solutal and thermal Rayleigh numbers are of the same order of magnitude appear worthwhile. The possibility of overstability requires the development of a better computer algorithm, which is capable of handling wide variations of parameters. If overstability occurs, the present results are only upper bounds for the stability-instability demarcation, i.e., although instability certainly occurs if $c_{\infty}>c_{\infty}^{* *}$ it may occur for even smaller values. This study has been concerned with the criteria for the onset of convective and morphological instabilities. Of great interest is the effect of convection on interface shape and the resulting solute distribution in the solidified material. A limited amount of information on this can be obtained from a linear analysis, e.g., the wavelength at the onset of instability. A nonlinear analysis is necessary for understanding the effects of fluid flow on solute distribution. In the near future we will further investigate the onset of instability for a wider range of parameters and develop improved computer algorithms. 


\section{EXPERIMENTAL INVESTIGATION}

\section{Experimental Procedures}

The procedures of sample preparation, directional solidification and sample characterization, which are being used to determine the effects of solutal convection on the microstructure and macrosegregation of off-eutectics, are reported in the following three sections.

Sample Preparation - Half kg alloy melts were prepared from 99.999 pct pure $\mathrm{Sn}$ and $\mathrm{Pb}$ in evacuated and sealed silica crucibles. After homogenization at 500C, crucibles were lowered quickly into $\mathrm{H}_{2} \mathrm{O}$ to prevent any macrosegregation in the ingots. Ingots were swaged into 1 cm diameter rods, from which 12 gm pieces were cut for each directional solidification tube. Five different alloys were prepared: Sn-35 w/o $\mathrm{Pb},-38.1 \mathrm{w} / \mathrm{O} \mathrm{Pb}$ (the eutectic), $-41 \mathrm{w} / \mathrm{o} \mathrm{Pb},-44 \mathrm{w} / \mathrm{o} \mathrm{Pb},-62 \mathrm{w} / \mathrm{o} \mathrm{Pb}$. Wet chemical analysis using a titration method yielded actual compositions of $34.7,37.8,40.7,43.7,61.1$.

The samples for directional solidification are contained in $3 \mathrm{~mm}$ ID, $4.2 \mathrm{~mm}$ OD silica tubes. In order to fill such thin tubes with alloy without bubbles the following method is followed. A tube is prepared with a $1 \mathrm{~cm}$ diameter $x 3 \mathrm{~cm}$ long silica cup at the top with a $0.4 \mathrm{~mm}$ orifice between the cup and tube. The lower half of the tube is coated with colloidal graphite and carefully dried. A thermocouple assembly is inserted through a graphite plug at the bottom of the tube and cemented in place with epoxy to form a vacuum tight seal. The 
assembly consists of a commercially available thermocouple contained in a $0.25 \mathrm{~mm}$ diameter stainless steel sheath coated with boron nitride. The top $1 \mathrm{~cm}$ of the thermocouple is exposed and the lower portion is held rigid by a $0.75 \mathrm{~mm}$ diameter $\mathrm{Al}_{2} \mathrm{O}_{3}$ tube.

The tube is then inserted into a simple vacuum furnace with only the cemented joint outside the furnace and a $12 \mathrm{gm}$ alloy slug in the cup. After evacuation the alloy charge is melted and the chamber is vented with Ar. This casts the alloy down over the thermocouple and fills the tube up to a level of about $18 \mathrm{~cm}$. The chamber is quickly filled with $\mathrm{H}_{2} \mathrm{O}$ to freeze the sample from bottom up to prevent shrinkage porosity.

Directional Solidification - The design of the directional solidification furnace is similar to that of Mollard and Flemings [4]. Briefly, it consists of a bifilar wound cylindrical resistance heating element with upper and lower sections and a circulating water cooled chill zone. The silica tube containing the alloy is pulled down through the chill sealed by O-rings at constant velocity to accomplish directional solidification. The cooling water comes directly in contact with the silica tube. The furnace is powered by an AC constant voltage power supply and the water is cooled by a heat exchanger. The vertical drive is powered by a synchronous motor with a multi-speed gear box. Speeds from $0.5 \mathrm{\mu m} / \mathrm{sec}$ to $0.5 \mathrm{~mm} / \mathrm{sec}$ are possible. The tube is attached to the pull shaft using a device which permits the sample to be suddenly pulled down about $3 \mathrm{~cm}$ to quench the liquid solid interface. 
The furnace is designed so that a permanent magnet with a pole diameter of $8 \mathrm{~cm}$ and a pole separation of $11 \mathrm{~cm}$ can be slid around the furnace to apply a 1 kilogauss horizontal magnetic field. Alternately, an $11 \mathrm{~cm}$ diameter solenoid can also be placed over the furnace-chill to apply a 700 gauss vertical magnetic field. The vertical field should be the most effective in preventing convection [39]. These magnetic fields will be used in future work under this contract.

A specific directional solidification experiment is conducted in the following manner. A tube filled with the desired alloy is inserted through the chill, with the alloy completely down in the chill section. A vacuum is pulled on the tube from the top. The furnace temperature is equilibrated for several hours. The temperature of the lower winding is used to control the temperature gradient. The sample is then run up quickly into the furnace melting about $12 \mathrm{~cm}$ of alloy. At this point the top $1 \mathrm{~cm}$ of the thermocouple is in the liquid. The tube is backfilled with Argon and temperatures are allowed to stabilize for about 20 minutes. The tube is then pulled down at the desired rate until about $6 \mathrm{~cm}$ have solidified. The remainder of the sample is then quenched delineating the liquid-solid interface in the microstructure. The thermocouple in the alloy is used to determine the temperature gradient in the liquid. The silica is removed with HF acid and the specimens are mounted in a cool-setting plastic for characterization. Characterization - Samples are routinely examined in a longitudinal section (parallel to the solidification direction) using standard 
metallographic techniques. When necessary transverse sections are also taken. For each experiment the shape of the liquid-solid interface is examined to be certain that heat flow was unidirectional. Whether the structure is dendritic, lamellar, or whether there are any signs of growth rate fluctuations is also determined.

As mentioned previously in this report, Verhoeven [7] has shown that considerable macrosegration occurs in directionally solidified offeutectics when solutal convection occurs. This macrosegregation is almost impossible to detect by looking at microstructure alone. Therefore for the present work it was desirable to measure the concentration as a function of distance along the growth direction in the directionally solidified material. Since we are interested in macrosegregation, this concentration must be averaged over the two phase structure and over as much of the entire cross section of the sample as possible. For this purpose a local energy dispersive $x$-ray fluorescence technique is being employed. This technique differs from ordinary $x$-ray fluorescence techniques in one important way: diffraction from a perfect Si crystal is employed to obtain a small monochromatic $x$-ray beam to excite fluorescence from the sample.

AgKc: radiation from a small focus conventional tube passes through a vertical slit $(0.3 \mathrm{~mm})$ and a horizontal slit $(1 \mathrm{~mm})$ which $\mathrm{sit} 20 \mathrm{~cm}$ from the source, and diffracts from the (220) plane of a perfect Si crystal in the reflection geometry (see Fig 2). The diffracted beam then passes through a wide vertical slit (1 $\mathrm{mm}$ ) and strikes the sample to be analyzed. This beam is $3 \mathrm{~mm}$ high and $0.5 \mathrm{~mm}$ wide at the sample 
position. The sample, which is sectioned longitudinally to the growth direction, is placed with the growth axis horizontal and perpendicular to the $x$-ray beam. The sample can be translated to perform a profile in the growth direction. Fluorescence from the sample is detected by a Si(Li) solid-state detector. The $10 \mathrm{~mm}$ diameter detector window is placed $40 \mathrm{~mm}$ from the sample surface and such that the scattering angle to the centerline of the detector is $135^{\circ}$. The $\mathrm{x}$-ray tube is operated at $55 \mathrm{kev}, 15 \mathrm{ma}$. This voltage is sufficiently high to also excite the (440) diffraction from the Si so that the collimated beam contains $22.1 \mathrm{kev}$ and $44.2 \mathrm{kev}$ components. This is done in order to excite Snka at $25.3 \mathrm{kev}$. However, at the present time only data from the $\mathrm{Pb}$ peaks is being used. The signal from the detector is processed by a multi-channel analyzer to form the x-ray emission spectrum from a position in the sample. A spectrum from a $\mathrm{Sn}-44 \mathrm{w} / \mathrm{O} \mathrm{Pb}$ alloy is shown in Fig 3. The spectrum in digital form can be transfered to a computer for analysis. The integrated intensity is obtained for the $\mathrm{PbL}, \mathrm{SnK}$ and TDS (thermal diffuse scattering from the incident beam peak). The latter serves as a monitor for the intensity of the incident beam. The ratio of $\mathrm{PbL}$ counts to TDS serves as a measure of the fraction of $\mathrm{Pb}$ in the sample. Results for runs on three different compositions are shown in Table 4. At this point, composition resolution of $0.5 \mathrm{w} / \mathrm{O} \mathrm{Pb}$ is possible. Further improvements are expected. In order to determine the effective size of the incident beam, spectra were collected from various positions across a planar interface 
separating a piece of $\mathrm{Au}$ from a piece of $\mathrm{Pb}$. The ratio of La counts to TDS counts for $\mathrm{Au}$ and $\mathrm{Pb}$ uncorrected for background are shown in Fig 4. This implies the effective size of the beam is $0.5 \mathrm{~mm}$; i.e., less than $10 \%$ of the counts come from a region wider than $0.5 \mathrm{~mm}$.

The reasons for using this particular technique are two-fold. First, previous electron microprobe analyses $[40]$ of composition profiles in two-phase structures have given very poor results presumably due to the very shallow penetration $(\curvearrowright 1 \mu \mathrm{m})$ of an electron beam into the alloy. The use of $x$-ray radiation for the incident beam greatly increases the penetration; as examples, in pure $\mathrm{Pb}$ the characteristic penetration depth $\left(\frac{1}{\mu}\right)$ where $\mu$ is the linear absorption coefficient for $\operatorname{AgK} \alpha$ is $10 \mu \mathrm{m}$ and in pure $\mathrm{Sn}$ it is $60 \mu \mathrm{m}$. The higher penetration of the $x$-ray radiation is necessary since the lamellar spacings are of the order of $5 \mathrm{\mu m}$. Second, it is necessary for this application to have an incident $x$-ray beam which is less than $1 \mathrm{~mm}$ wide and which is monochromatic (or bichromatic). The small size is required so that the profile can be measured with good spatial resolution and the monochromatic beam is needed to reduce the background to permit good composition sensitivity. X-ray energy dispersive techniques not employing diffraction from a first crystal are typically used for bulk analysis.

Further improvement will be obtained using a Ge crystal to increase the diffracted intensity. Also by using a dual slit, a piece of the 
diffracted beam will be monitored by a proportional counter. This should prove supérior to using TDS as a monitor because a larger number of counts can be collected. This will improve the counting statistics and improve the composition sensitivity.

\section{Results}

The experimental accomplishments of the first year's work under this contract consist primarily of the development of the specific procedures of sample preparation, directional solidification and sample characterization described in the previous sections. Using these procedures, significant advances are expected in the future. A few preliminary results obtained from $\mathrm{Pb}-\mathrm{rich}, \mathrm{Pb}-\mathrm{Sn}$ off-eutectics will be described.

More than 20 directional solidification experiments have been performed on the 38.1, 41 and $44 \mathrm{w} / \mathrm{O} \mathrm{Pb}$ alloys at solidification velocities from $0.9 \mathrm{\mu m} / \mathrm{s}$ to $32 \mathrm{\mu m} / \mathrm{s}$. Many of these samples were solidified to check the furnace temperature stability and drive mechanism and to determine the relation between furnace temperature and temperature gradient in the liquid at the interface. A problem encountered and solved this year is demonstrated in Figure 5. This micrograph shows a growth rate fluctuation during the directional solidification of an off-eutectic composite. This type of fluctuation was found not to be due to temperature instability or drive mechanism but by sticking of the alloy to the silica tube as described by Verhoeven and Gibson [41]. These fluctuations were eliminated by using a colloidal graphite coating on the interior of the silica tube. 
An interesting preliminary observation has been made. When samples with compositions on the $\mathrm{Pb}-$ rich side of the eutectic (41 w/o $\mathrm{Pb}$ and 44 w/o $\mathrm{Pb}$ ) are solidified at velocities high enough to ensure dendritic solidification, the solidification starts out dendritic but changes to composite after solidication distances of the order of centimeters. For example, a 41 w/o alloy solidified at a temperature gradient of $280 \mathrm{~K} / \mathrm{cm}$ and a velocity of $31.7 \mu \mathrm{m} / \mathrm{sec}$ became composite after a distance of $2 \mathrm{~cm}$. Figure 6 shows the gradual disappearance of the dendritic structure in a Pb-rich off-eutectic directionally solidified sample. The composition of a composite region of such a sample determined by wet chemical analysis was $39.6 \mathrm{w} / \mathrm{O} \mathrm{Pb}$; whereas the bulk alloy composition was $40.7 \mathrm{w} / \mathrm{O} \mathrm{Pb}$. The change in structure down the length of a directionally solidified sample is shown in higher magnification in Figure 7 and may be typical of macrosegregation in dendritic castings caused by solutal convection [11]. In the future, measurement of composition profiles using the $x$-ray method will confirm or deny this conjecture of macrosegregation.

Future efforts will concentrate on an accumulation of reproducible data on the microstructure and the macrosegregation present in samples solidified under various conditions thought to produce solutal convection. The effect of the magnetic field on the suppression of these flows will then be initiated. 


\section{Acknowl edgements}

We acknowledge the substantial contributions of Professor Robert F. Sekerka of Carnegie-Mellon University and Consultant to the National Bureau of Standards to the theoretical part of this research. We would also like to thank J. R. Carruthers, D. T. J. Hurle, M. Kuriyama and R. G. Rehn for helpful discussions. 
References

[1] J.R. Carruthers, Preparation and Properties of Solid State Materials, Vol. 3, Eds. W. R. Wilcox and R. A. Lefever (Marcel Dekker, Inc., New York, 1977) p. 1.

[2] D。 T. J. Hurle, Current Topics in Materials Science, Vo1. 2, Eds. E. Kaldis and H. J. Scheel (North-Holland, Amsterdam, 1977) p. 549 .

[3] R. M. Sharp and A. Hellawel1, J. Crystal Growth 12, 261 (1972).

[4] F. R. Mo lard and M. C. Flemings, Trans. AIME 239, 1534 (1967).

[5] J. D. Holder and B. F. Oliver, Met. Trans 5, 2423 (1974).

[6] K. G. Davis and L. M. Hogan, unpublished results shown in K. G. Davis and P. Fryzuk, Can. Met. Quart, 10, 273 (1971).

[7] J. D. Verhoeven, K. K. Kingery and R. Hofer, Met. Trans. 6 B, $647(1975)$.

[8] J.A. Burton, R. C. Prim and W. P. Slichter, J. Chem. Phys. $\underline{21}, 1987$ (1953).

[9] J. D. Verhoeven, J.C. Warner and E. D. Gibsor, Met. Trans. 3 , 1437 (1972)

[10] L. Vandenbulcke and G. Vuillard, J. Crystal Growth 36, 47 (1976).

[11] N. Streat and F. Weinberg, Met. Trans. 5, 2539 (1974) and references therein.

[12] S. Chandrasekhar, Hydrodynamic and Hydromagnetic Stability (Oxford, London, 1961).

[13] J. S. Turner, Buoyancy Effects in Fluids (Cambridge, London, 1973). 
[14] D. D. Joseph, Stability of Fluid Motions I and II (SpringerVerlag, Berlin, 1976).

[15] G. Z. Gershuni and E. M. Zhukhovitskii, Convective Stability of Incompressible Fluids (Keter, Jerusalem, 1976).

[16] C. Normand, Y. Pomeau and M. G. Velarde, Rev. Mod. Phys. 49, $581(1977)$.

[17] J. S. Turner, Ann. Rev. Fluid. Mech. 6, 37 (1974).

[18] R. S. Schechter, M. G. Velarde and J. K. Platten, Adv. Chem. Phys. 26, 265 (1974).

[19] M. E. Stern, Tellus 12, 172 (1960).

[20] R。L。Sani, Ph.I). dissertation, University of Minnesota, 1963.

[21] D. A. Nield, J. Fluid Mech.29, 545 (1967).

[22] P. G. Baines and A. E. Gill, J. Fluid Mech. 37, 289 (1969).

[23] G. Veronis, J. Mar. Res. 23, 1 (1965).

[24] R. L. Sani, A. I. Ch. E. J. 11, 971 (1965).

[25] G. Veronis, J. Fluid Mech. 34, 315 (1968).

[26] J。M. Straus, J. Fluid Mech. 56, 353 (1972).

[27] H. E. Huppert and D. R. Moore, J. Fluid Mech. 78, 821 (1976).

[28] R. F. Sekerka, Crystal Growth: An Introduction, Ed. P. Hartman (North-Holland, Amsterdam, 1973) p. 403.

[29] R. T. Delves, Crystal Growth, Vol. 1, Ed. B. R. Pamplin (Pergamon, Oxford, 1974) p. 40.

[30] H. B. Keller, Numerical Methods for Two-Point Boundary Value Problems (Blaisdel, Waltham, MA 1968). 
[31] H. B. Keller, Numerical Solution of Two Point Boundary Value Problems (Society for Industrial and Applied Mathematics, Philadelphia 1976)

[32] D. P. Chock and R. S. Schechter, Phys. Fluids 16, 329 (1973).

[33] D. P. Chock and C.-H. Li, Phys. Fluids 18, 1401 (1975).

[34] L. F. Shampine and M. K. Gordon, Computer Solution of Ordinary Differential Equations - The Initial Value Problem (W. H. Freeman, San Francisco, 1975).

[35] K. M. Brown, SIAM J. Numer. Anal . 6, 560 (1969).

[36] b. M. Gay, CNA-109 (Center for Numerical Analysis, The University of Texas at Austin) Dec. 1975 .

[37] W. H. Reid and D. L. Harris, Phys. Fluids 1, 102 (1958).

[38] E. M. Sparrow, R. J. Goldstein and V. K. Jonsson, J. Fluid Mech. $\underline{18}, 513(1964)$

[39] B. Lehnert and N. C. Little, Tellus 9, 97 (1957)。

[40] R. M. Sharp and A. Hellawel1, J. Crystal Growth 5, 155 (1969).

[41] J. D. Verhoeven and E. D. Gibson, J. Crystal Growth 10, 235 (1971) . 
Table 1. Values Used in Numerical Calculations

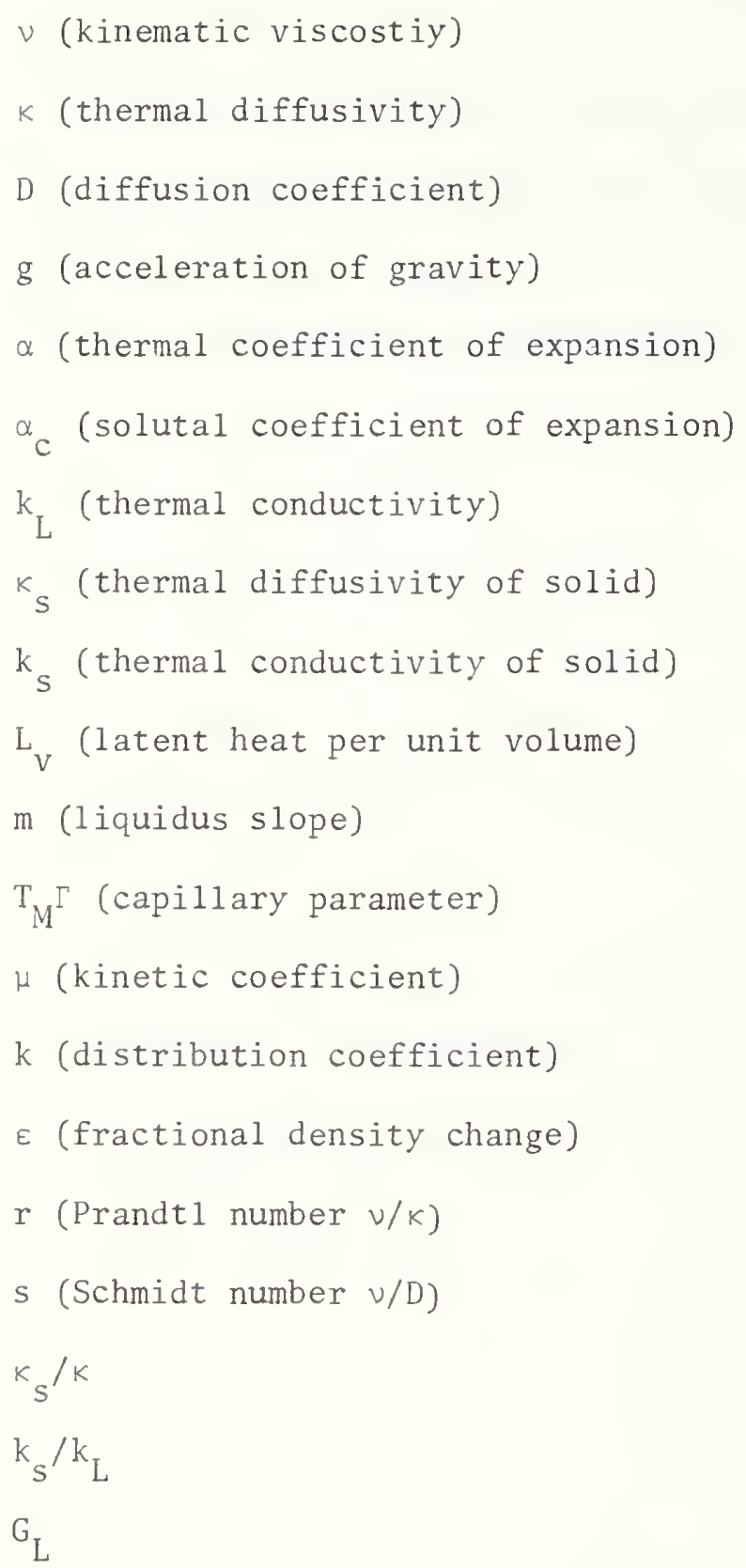

\begin{tabular}{|c|c|}
\hline $2.43\left(10^{-3}\right)$ & $\mathrm{cm}^{2} / \mathrm{s}$ \\
\hline .108 & $\mathrm{~cm}^{2} / \mathrm{s}$ \\
\hline $3.0\left(10^{-5}\right)$ & $\mathrm{cm}^{2} / \mathrm{s}$ \\
\hline 980.0 & $\mathrm{~cm} / \mathrm{s}^{2}$ \\
\hline $1.15\left(10^{-4}\right)$ & $K^{-1}$ \\
\hline $5.2\left(10^{-3}\right)$ & $(w / 0)^{-1}$ \\
\hline .159 & $\mathrm{~J} / \mathrm{cmKs}$ \\
\hline .202 & $\mathrm{~cm} /{ }^{2} \mathrm{~s}$ \\
\hline .297 & $\mathrm{~J} / \mathrm{cmKs}$ \\
\hline 256.0 & $\mathrm{~J} / \mathrm{cm}^{3}$ \\
\hline-2.33 & $K /(w / o)$ \\
\hline $1.0\left(10^{-5}\right)$ & $\mathrm{cmK}$ \\
\hline$\infty$ & $\mathrm{cm} / \mathrm{sK}$ \\
\hline 0.3 & \\
\hline 0 & \\
\hline $2.25\left(10^{-2}\right)$ & \\
\hline 81.0 & \\
\hline 1.87 & \\
\hline 1.87 & \\
\hline 200.0 & $\mathrm{~K} / \mathrm{cm}$ \\
\hline
\end{tabular}


Table 2. Comparison of Numerical Results with Previous Results

\begin{tabular}{|c|c|c|c|c|c|}
\hline Case $^{+}$ & $\omega$ & $\mathrm{ERR}^{++}$ & $\mathrm{R}$ & $\begin{array}{c}\mathrm{R} \\
\text { (previous) } \\
\end{array}$ & Reference \\
\hline 1 & 3.117 & $10^{-4}$ & $170 \% .35$ & 1707.762 & 37 \\
\hline 2 & 2.55 & $10^{-4}$ & 1295.67 & 1295.781 & 38 \\
\hline Case & $\omega$ & ERR & $\mathrm{R}+\mathrm{S}$ & $\begin{array}{c}\mathrm{R}+\mathrm{S} \\
\text { (previous) } \\
\end{array}$ & Reference \\
\hline 3 & 3.117 & $10^{-4}$ & 1707.8 & 1707.762 & 21,37 \\
\hline 3 & 3.117 & $10^{-4}$ & 1707.5 & 1707.762 & 21,37 \\
\hline 3 & 3.117 & $10^{-8}$ & 1707.762 & 1707.762 & 21,37 \\
\hline 3 & 8.0 & $10^{-6}$ & 7084.50 & 7084.51 & 21,37 \\
\hline 3 & 20.0 & $10^{-8}$ & 173573.47 & 173573.46 & 21,37 * \\
\hline 3 & 20.0 & $10^{-6}$ & 218625 & 218584.1 & $21,37 *$ \\
\hline 3 & 20.0 & $10^{-8}$ & 218584.1 & 218594.1 & $21,37 *$ \\
\hline 4 & $\pi /(2)^{\frac{1}{2}}$ & $10^{-6}$ & 657.577 & 657.5114 & 21 \\
\hline 4 & 10.0 & $10^{-8}$ & 943916 & 943864 & 21 \\
\hline 4 & 20.0 & $10^{-8}$ & 172785 & 172138 & 21 \\
\hline
\end{tabular}

${ }^{+}$Case 1 : rigid; $\hat{T}=0$ at $z=0$ and $z=1 ; \hat{C}=0$ for all $z$.

Case 2: rigid; $\hat{T}=0$ at $z=0$ and $(\partial \hat{T} / \partial z)=0$ at $z=1 ; \hat{C}=0$ for all $z$.

Case 3 : rigid; $\hat{T}=\hat{C}=0$ at $z=0$ and $z=1$.

Case 4: free; $\hat{T}=\hat{C}=0$ at $z=0$ and $z=1$.

rigid: $\hat{W}_{z}=\left(\partial \hat{W}_{z} / \partial z\right)=0$ at $z=0$ and $z=1$.

free : $\hat{W}_{z}=\left(\partial^{2} \hat{W}_{z} / \partial z^{2}\right)=0$ at $z=0$ and $z=1$.

${ }^{++}$ERR is an error tolerance in the code for solving the differential equations.

*alculated from formula of reference 37. 
Table 3. Bulk Concentrations, $c_{\infty}^{* *}$ at Which Convection First Occurs as a Function of Velocity V

\begin{tabular}{|c|c|c|c|c|c|}
\hline $\begin{array}{c}V \\
(\mu \mathrm{m} / \mathrm{s}) \\
\end{array}$ & $\begin{array}{r}c^{* *} \\
(w / 0) \\
\end{array}$ & $\begin{array}{r}\lambda \\
\mathrm{cm} \\
\end{array}$ & $\mathrm{D} \omega / \mathrm{V}$ & $\mathrm{S}^{-0}$ & $\begin{array}{l}c^{\infty} \\
w / 0 \\
\end{array}$ \\
\hline 1.0 & $3.16\left(10^{-4}\right)$ & 2.4 & 0.8 & 1390 & $5.7\left(10^{-1}\right)$ \\
\hline 2.5 & $4.10\left(10^{-4}\right)$ & 1.0 & 0.7 & 116.0 & $2.3\left(10^{-1}\right)$ \\
\hline 5.0 & $8.39\left(10^{-4}\right)$ & 0.6 & 0.6 & 29.6 & $1.1\left(10^{-1}\right)$ \\
\hline 10.0 & $3.10\left(10^{-3}\right)$ & 0.4 & 0.4 & 13.6 & $5.7\left(10^{-2}\right)$ \\
\hline 20.0 & $1.89\left(10^{-2}\right)$ & 0.26 & 0.36 & 10.4 & $2.8\left(10^{-2}\right)$ \\
\hline 40.0 & $1.86\left(10^{-1}\right)$ & 0.14 & 0.34 & 12.8 & $1.4\left(10^{-2}\right)$ \\
\hline 80.0 & $1.16\left(10^{-1}\right)$ & 0.0070 & 3.4 & 1.00 & $7.1\left(10^{-3}\right)$ \\
\hline
\end{tabular}

See Table 1 for values of physical quantities used in the calculations; $S^{\prime}=\left(g \alpha_{c} / \nu D k\right)(1-k) c_{\infty}^{* *}(D / V)^{3}$ and $c_{\infty}^{\prime \prime}=\left(\alpha G D / \alpha_{c} V\right)[k /(1-k)]$. For $c_{\infty}<c_{\infty}^{\prime \prime}$ the unperturbed density is a monotonically decreasing function of height. 
Table 4. Check on Reproducibility and Sensitivity of X-Ray Chemical Analysis Technique

\begin{tabular}{|c|c|c|c|c|c|}
\hline \multirow{2}{*}{$\begin{array}{l}\text { Analys is } \\
\text { No. }\end{array}$} & \multirow{2}{*}{$\begin{array}{c}\text { Composition } \\
w / 0 \mathrm{~Pb}\end{array}$} & \multicolumn{2}{|c|}{$\begin{array}{l}\text { Integrated Intensity } \\
\text { (counts) }\end{array}$} & \multirow{2}{*}{\multicolumn{2}{|c|}{$\begin{array}{c}\text { Ratio } \\
\text { PBL/TDS } \\
\end{array}$}} \\
\hline & & $\mathrm{PbL}$ & TDS & & \\
\hline 105 & 37.8 & 206,747 & 24,580 & 8.41 & \\
\hline 106 & 37.8 & 223,252 & 26,105 & 8.55 & $846+06$ \\
\hline 107 & 37.8 & 207,524 & 24,526 & 8.46 & \\
\hline 108 & 37.8 & 192,960 & 22,923 & 8.42 & \\
\hline 109 & 40.7 & 212,595 & 22,722 & 9.36 & \\
\hline 110 & 40.7 & 213,104 & 22,580 & 9.44 & $9.43+06$ \\
\hline 111 & 40.7 & 213,708 & 22,717 & 9.41 & \\
\hline 112 & 40.7 & 213,863 & 22,496 & 9.51 & \\
\hline 113 & 43.7 & 258,690 & 25,571 & 10.12 & \\
\hline 114 & 43.7 & 256,742 & 25,762 & 9.97 & \\
\hline 115 & 43.7 & 263,423 & 25,948 & 10.15 & \\
\hline 116 & 43.7 & 245,714 & 24,410 & 10.07 & \\
\hline 117 & 43.7 & 232,170 & 22,874 & 10.15 & $10.11 \pm .08$ \\
\hline 118 & 43.7 & 225,439 & 22,135 & 10.19 & \\
\hline 119 & 43.7 & 222,242 & 22,094 & 10.06 & \\
\hline 120 & 43.7 & 229,763 & 22,897 & 10.04 & \\
\hline 121 & 43.7 & 244,851 & 24,006 & 10.20 & \\
\hline
\end{tabular}

A $0.5 \mathrm{w} / \mathrm{O} \mathrm{Pb}$ change corresponds roughly to a $\frac{\mathrm{PBL}}{\mathrm{TDS}}$ ratio change of 0.13 . 


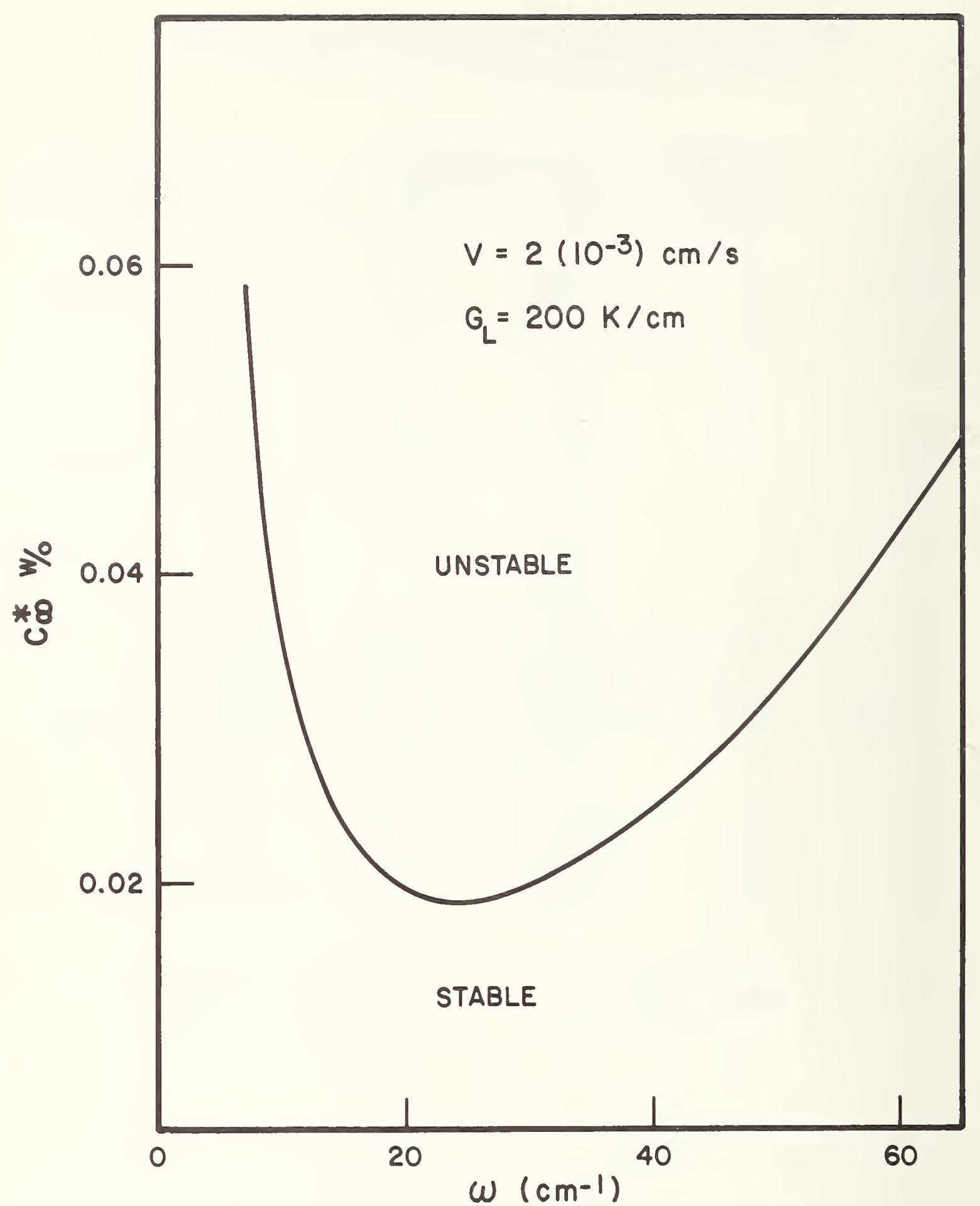

Fig. 1. The stability-instability demarcation as a function of the spatial frequency $w$ for a growth velocity of $2\left(10^{-3}\right) \mathrm{cm} / \mathrm{s}$ and a temperature gradient in the liquid of $200 \mathrm{~K} / \mathrm{cm}$. For values of the bulk concentration $c_{\infty}$ above the curve, the system is unstable with respect to disturbances of wavelength $2 \pi / \omega$. For $c_{\infty}<0.0189 \mathrm{w} / 0$, the fluid is stable with respect to all small $1^{\infty}$ perturbations. 


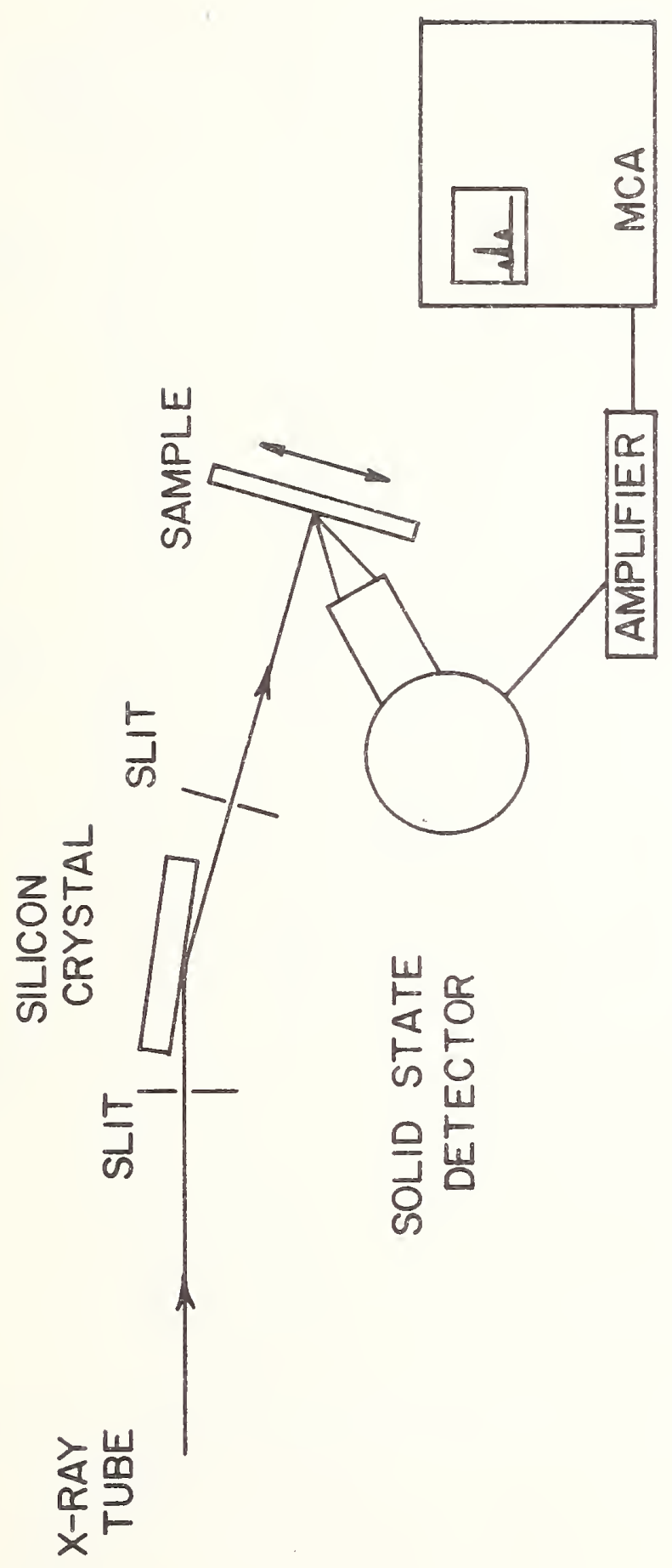

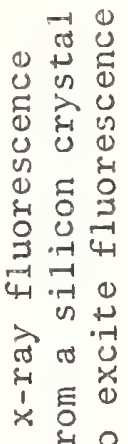

(1) 幽 옹

礼范

ध्र.

(1)

का

$\rightarrow$ तो

0 4

合然 $x$

-

¿.

(1) i

$\rightarrow$ 的

ขิ

$\rightarrow$ 둥

4

- $\rightarrow$

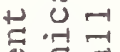

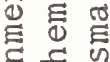

हू है

- $\rightarrow$ क्

का

तี

- $\begin{gathered}0 \\ 0\end{gathered}$

सכ年

용

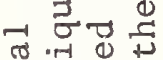

起

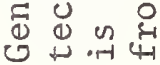

$\dot{N}$

os

. 


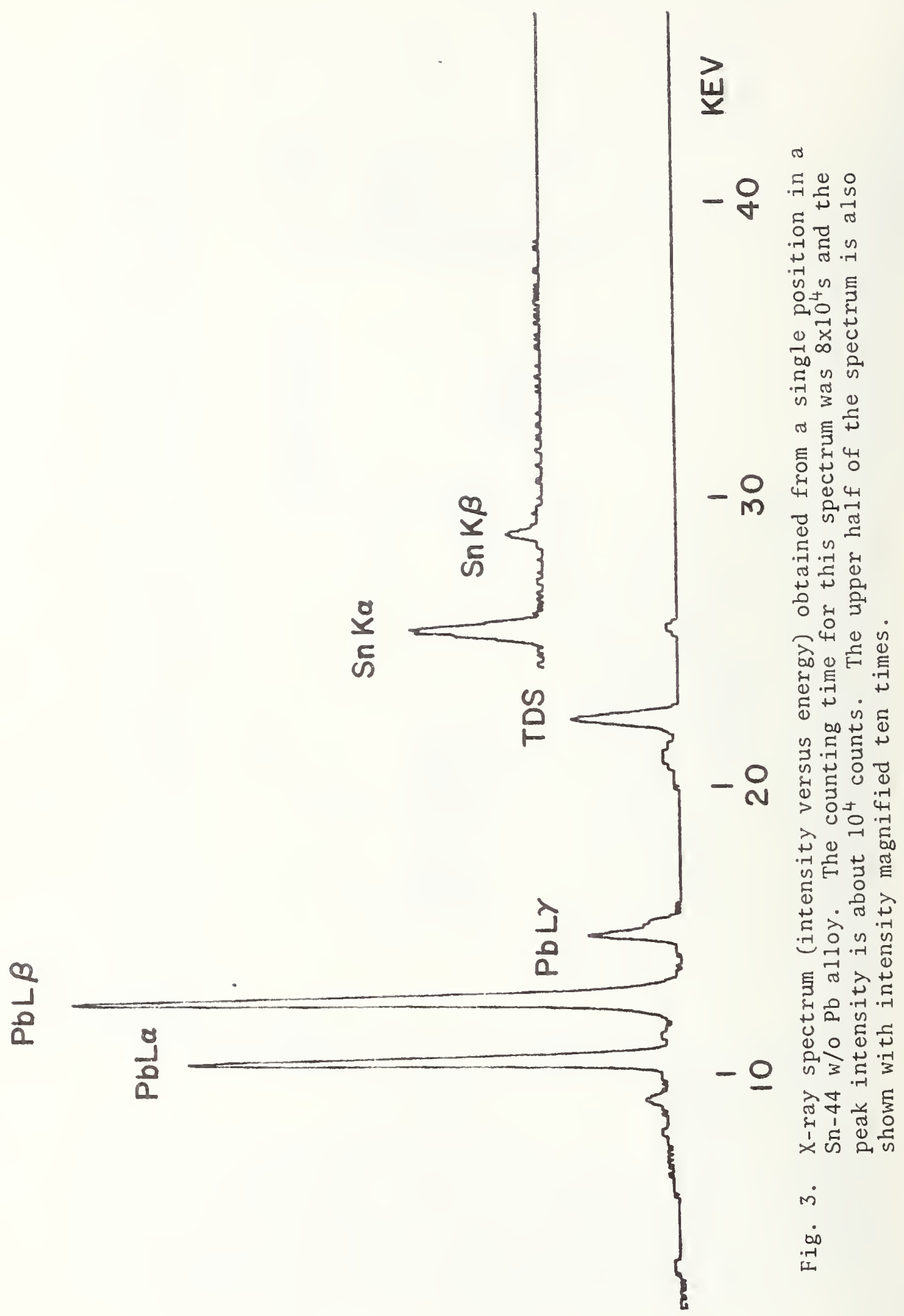




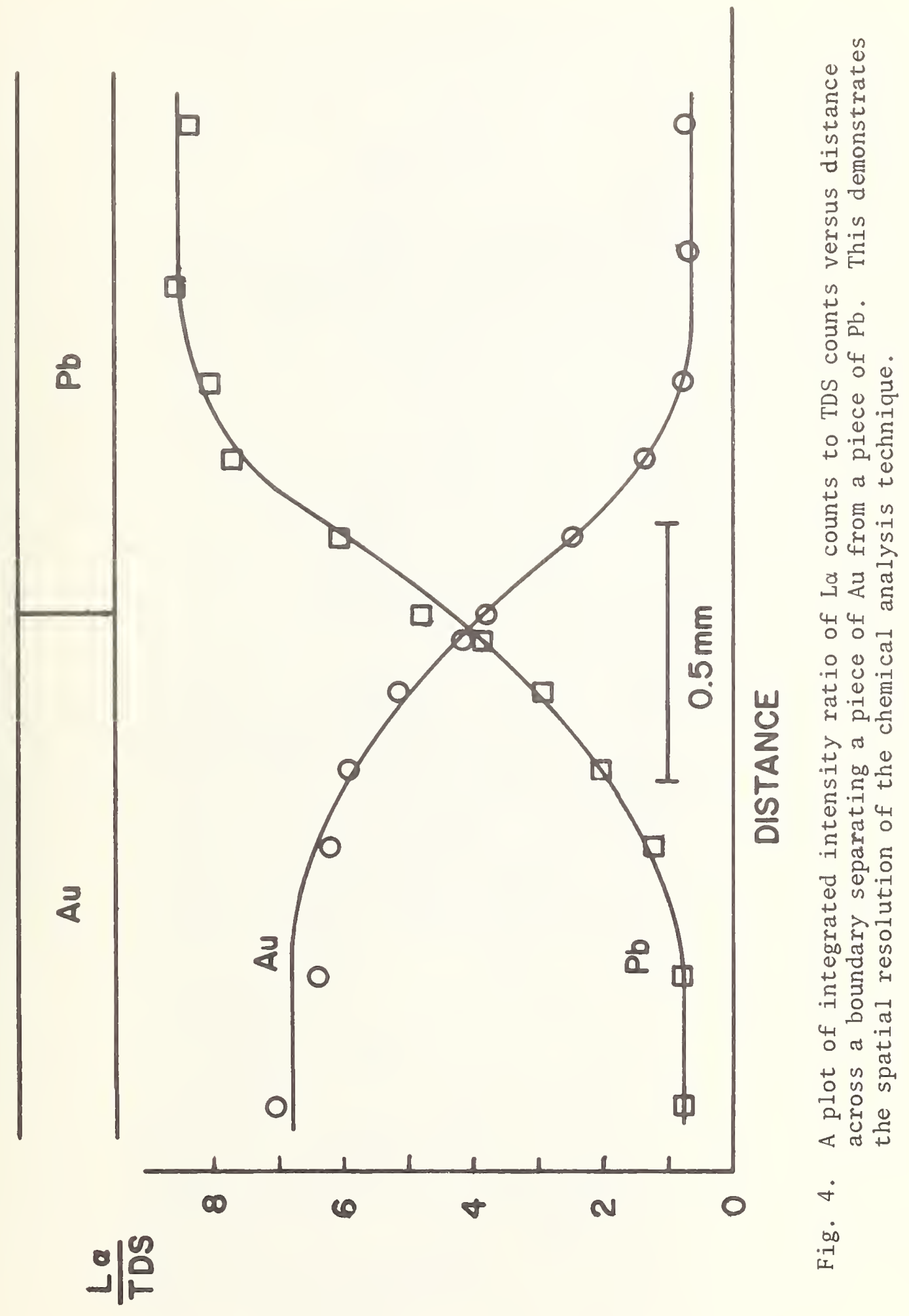




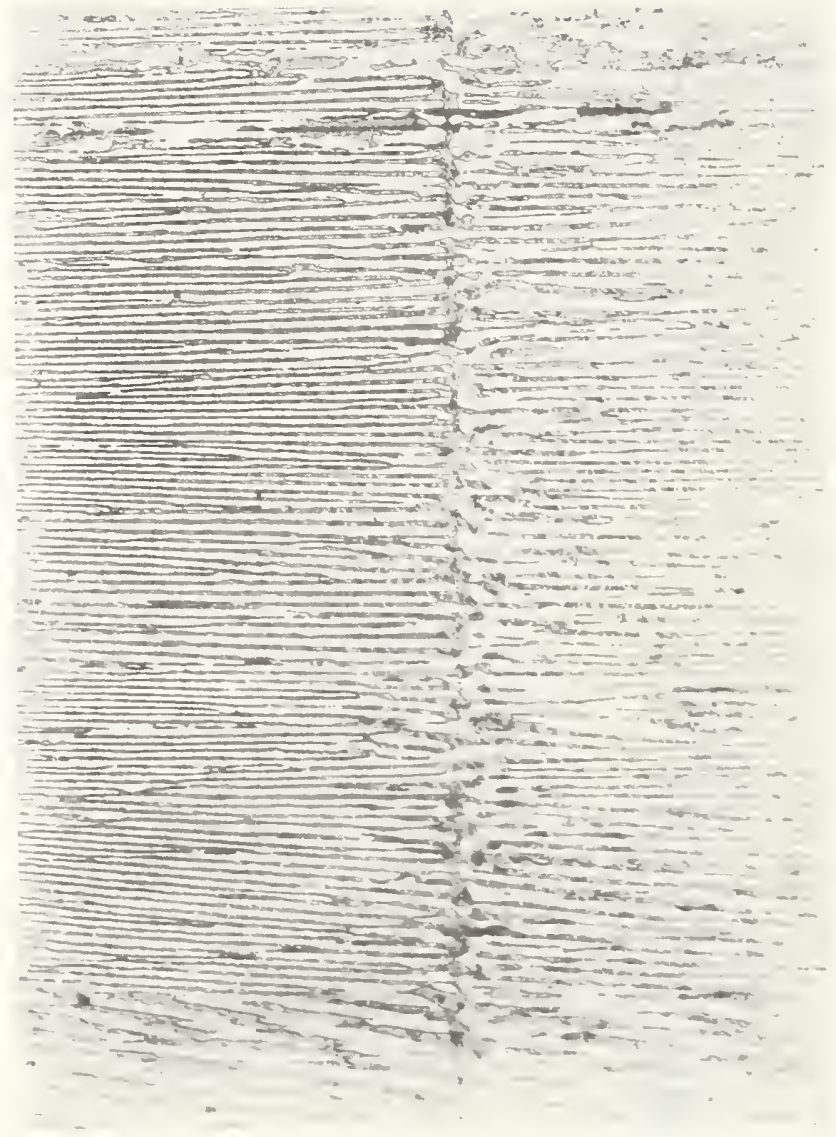

Fig. 5. Longitudinal section of a growth rate fluctuation caused by sticking of the alloy to the silica tube during directional solidification. The $\mathrm{Pb}-\mathrm{rich}$ phase is dark and the Sn-rich phase is light. Optical micrograph. Magnification 256 times 


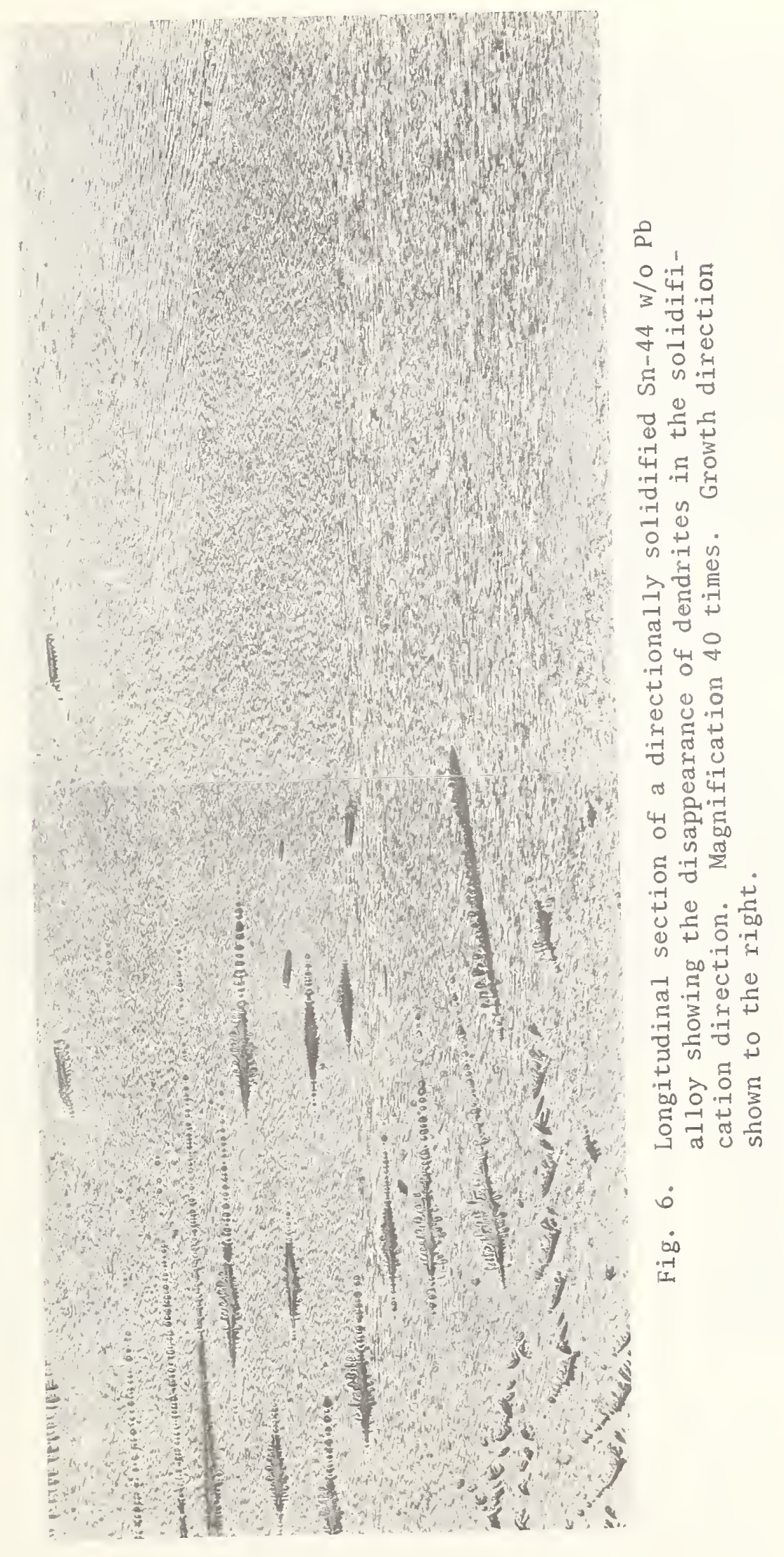



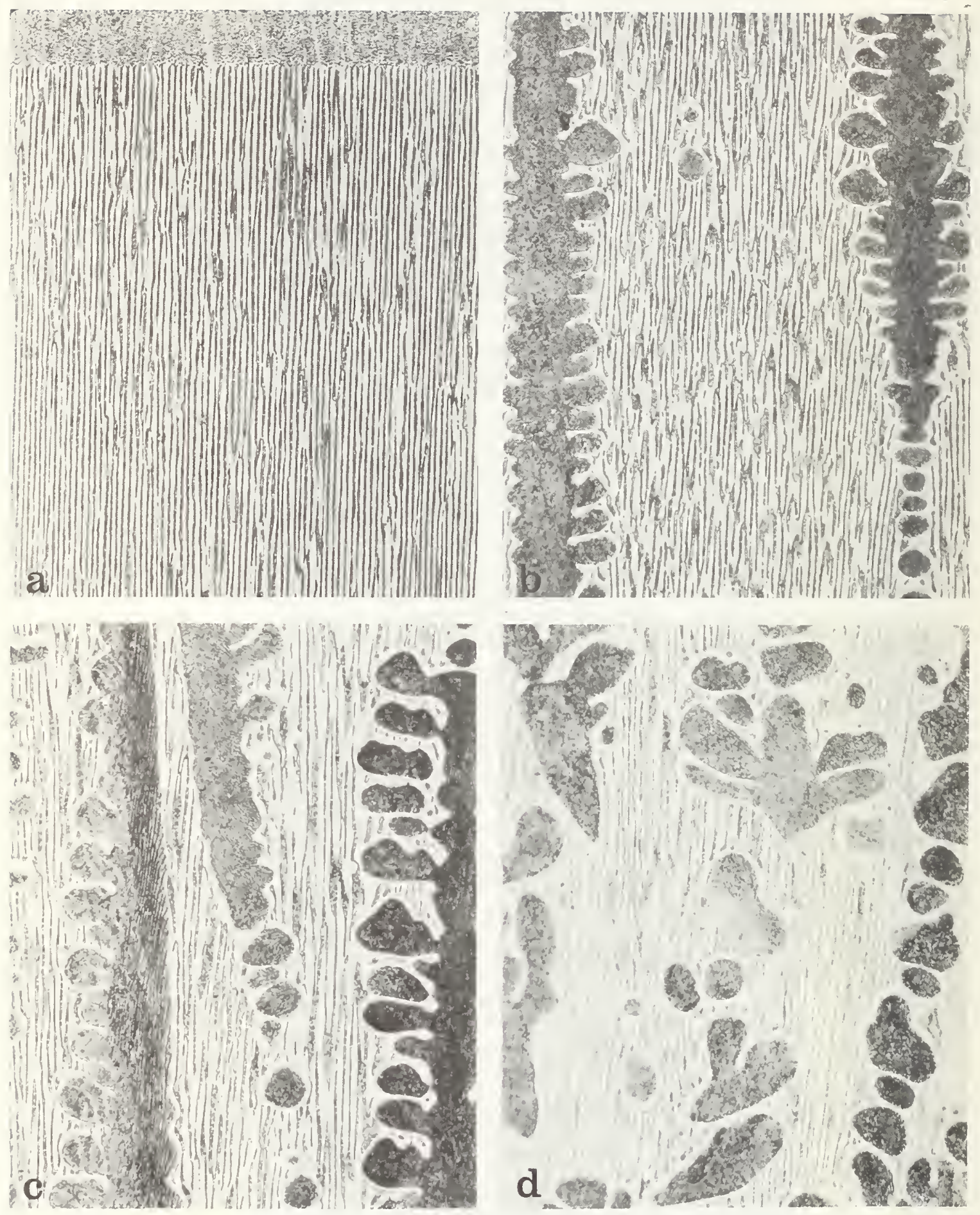

Fig. 7. Longitudinal section of a directionally solidified Sn-44 w/o $\mathrm{Pb}$ alloy showing the change in structure in the solidification direction.

(a) Quenched liquid-solid interface, $4.2 \mathrm{~cm}$ solidified, (b) $3.0 \mathrm{~cm}$ solidified, (c) $1.7 \mathrm{~cm}$ solidified, (d) $0.2 \mathrm{~cm}$ solidified. Growth direction shown vertically. Magnification 256 times. 
Determination of Crystal Perfection

M. Kuriyama and H. E. Burdette

Metal Science and Standards Division

Center for Materials Science

\section{Summary}

The objective of this year's task is shifted from the actual crystal growth on Earth to helping NASA establish methods for evaluating spacegrown crystals with reference to nearly perfect crystals grown on Earth. For this purpose our intention is to perform Asymmeric (Double) Crystal Topography (ACT) on a real-time basis, so that any laboratory can evaluate crystal perfection routinely with the same accuracy using an ACT camera. In this report Section II includes the crystal characterization work on nickel single crystal growth with emphasis on the interpretation of observed imperfection images including a new rule of image invisibility. Section III covers our activities on real-time imaging, which are aimed toward improvement of resolution and brightness. 


\section{INTRODUCTION}

The previous objectives dealt with the assessment of crystal perfection as crystal growth conditions were deliberately changed, so as to obtain the relationship between resultant crystal perfections and crystal growth conditions $[1-3]$. In order to do this it was necessary to introduce a new technique for the evaluation of crystal perfection by x-ray diffraction topography, namely Asymmetric Double Crystal Topography (ACT) ${ }^{[1,4-7]}$. With this technique, we were able to evaluate the crystal perfection of a large number of Czochralski grown crystals (about 20 boules a year and more than 50 crystal slices) routinely but more accurately than the ordinary topographic methods.

The objective of this year's task is different from those of previous years. This year's goal is shifted from the actual crystal growth on Earth to helping NASA establish methods for evaluating space-grown crystals in comparison with crystals grown on Earth. Our intention is to perform ACT on a real-time basis so that any laboratory can evaluate crystal perfection routinely with the same accuracy using an ACT camera.

In the past, we demonstrated that ACT is best suited to the characterization and assessment of crystals and is superior to other well-known topographic techniques, such as the Berg-Barrett technique and Lang techniques $[7,8]$. This is because the ACT system is more sensitive to strains and imperfections in crystals than the other methods. A well-considered use of the x-ray diffraction effect makes it possible to obtain improved $x$-ray optical resolution and strain- 
sensitivity. Although this ACT system is versatile and simple to operate in that it requires neither a-micro-focus $x$-ray source and generator nor a scanning device, this system still suffers from one problem common to other topographic systems. This problem is that it takes a long exposure to record high resolution diffracted images, in which all the information necessary to characterize the crystal is delivered. This cumbersome problem deters many people from characterizing their crystals accurately.

In this task, we aim at obtaining improved real-time imaging systems with sufficient resolution and intensity-sensitivity。 These systems, once successful, will be used not only with the ACT system but also with $\mathrm{x}$-ray or $\gamma$-ray radiographic systems for material flaw inspection. Since the work on this task was at a reduced level this year, the results reported here are by no means final and should be considered as a starting point for further work。

Section II includes the crystal characterization work on nickel single crystal growth, which has thus far been unreported. Section III covers our activities on real-time imaging.

\section{CHARACTERIZATION OF CRYSTAL PERFECTION}

In $x$-ray topography, the visibility or invisibility of imperfection images is quite important for the characterization of crystal imperfections. The direction (and sometimes also the magnitude) of atomic displacements can be determined by just looking for the existence or non-existence of images. One of the unique features of the ACT system 
enables us to observe imperfections deep in the interior of quite thick ( $1 \mathrm{~mm}$ ) crystals. In the $x$-ray topography of thin crystals kinematical scattering processes dominate the formation of topographic images. By contrast, in the $x$-ray topography of thick crystals, the conditions of invisibility differ considerably, depending upon the nature of imperfections [6]. Here the product of thickness and linear absorption coefficient, $\mu \mathrm{L}$, is greater than 10. These conditions had never been studied before the ACT system was fully developed. The recent availability of synchrotron radiation will now provide the same opportunity as the ACT system.

We continued the characterization of nickel single crystals grown under different conditions. Since nickel is ferromagnetic, magnetic domains form in it. The magnetic domain walls between the domains are usually strained due to a magnetostriction; in other words, the domain walls can be thought of as crystal imperfections. When magnetic domain walls extend all the way through a crystal, and the crystal is thick, topographic images are usually produced by the domain walls. These images are called disruption images or shadows.

We have observed a new rule for the visibility or invisibility of these domains ${ }^{[9]}$. These rules relate crystal and defect orientations to diffraction condition, as described in reference 9. This new rule is, in general, applicable to a non-isolated thin planar defect which has an extremely regular atomic displacement. One such defect could be a stacking fault. 
The methods of interpreting topographic images in ACT have been explained elsewhere ${ }^{[10,11]}$ through the application to nickel crystals. When bulk crystals are used for the true assessment of crystal imperfections, the interpretation of images obtained with the ACT systems or synchrotron radiation requires different rules from the familiar ones for the ordinary Lang topography. With ACT system, for example, it is possible to identify crystal imperfections whose identities would not be uniquely determined by ordinary Lang techniques.

\section{III。 RESOLUTION IMPROVEMENT IN REAL-TIME IMAGING}

For the rapid and accurate assessment of crystal perfection, an improvement in resolution of a real-time imaging system is required without loss of image brightness. As in ordinary optics, two principal problems will be involved to achieve improved resolution: one is the collimation of incoming beam, and another is the spatial resolution on an imaging detector. If one could make the size of the beam source truly a mathematical point, the first problem would be solved. However, this is not possible. One has to devise a method of controlling the spatial size and angular divergence of the incident beam regardless of the size of the beam source -- this is the problem of collimation.

We deal with two objects each of which has a finite size and which are located a given distance apart. Tentatively, resolution will be defined by the minimum distance between the centers of these two objects before the images from the two objects start overlapping in the imaging detector. Before describing the method used in the current work, other 
methods of improving angular resolution will be described for comparison. It is concluded that the method being developed in the current work provides major advantages over these other methods.

\section{Collimation}

The earliest attempts at spatial collimation of an $x$-ray beam consisted of an arrangement of single slits to define beam direction, sometimes followed by Bragg reflection by a crystal. The beam collimated in this way was, generally, quite small. Soller ${ }^{[12]}$ first suggested collimating an x-ray beam by using multiple long foils, each spacing defining a narrow beam divergence, over a rather large area. It is interesting to note that Soller's original plan was to study microscopic changes due to thermal, mechanical, or other physical influences on materials. The first Soller slits were made with lead foils, with a length to spacing ratio of $166: 1$. Although lead is not the ideal material for Soller slit foils because of its mechanical properties, it does illustrate several other important considerations in the manufacture of this device. It is very important to avoid $\mathrm{K}$-fluorescence and surface reflection by the foils. There are clearly two ways to avoid the first problem: either the foil materials must have a higher K-excitation energy than the energy of the penetrating radiation, or they must have such a low excitation energy that, although much fluorescence is present, it is absorbed either in air or the detector window or can be discriminated by the pulse height analysis system. The best collimation will be achieved if the foil material can be chosen such that it has an absorption edge 
near the energy of the x-ray beam. The problem of surface reflection on the foils has been at least partially solved by roughening the foils with emery, with a reported ${ }^{[13]} 20 \%$ increase in resolving power for the case of FeKa x-rays collimated by $\mathrm{Al}$.

The common Soller slit ratio in use today is $30: 1$, resulting in an angular divergence of about $2^{\circ}$, compared to Soller's $1 / 3^{\circ}$. The ultimate collimation the device can achieve is the order of 1 to 10 arc minutes angular divergence。

Another collimating device, originally designed to study grain boundaries, inclusions and details of histological specimens, utilized surface reflections in order to improve the ratio of transmitted to incident flux. This device consisted of bundles of glass capillaries [14] from $2-12 \times 10^{-6} \mathrm{~m}$ in diameter and $0.5-1.0 \times 10^{-2} \mathrm{~m}$ long. Although the apparent "best case" collimation is of the order of 1 arc minute, the angular divergence is significantly greater because of the reflections. One can make a similar statement where a single wedge-shaped glass arrangement is used to collimate $\mathrm{CuK} \alpha \mathrm{x}$-rays. An actual collimation of 15 arc minutes was achieved, with an ultimate limit of 5 arc minutes predicted $^{[15]}$.

A novel approach to the mechanical collimation of $x$-rays -- the multiple slit array[16] -- eliminates entirely the internal reflection of off-axis radiation. This device was conceived for $x$-ray astronomy, where it is desirable to have a large percent transmission over a very narrow angle so that $x$-ray fluxes from neighboring sources in the galaxy 
could be resolved. In this collimator, identical grids, which are prepared from a master by precision photomicrographic techniques, are spaced in a progressive pattern. That pattern is designed, based on simple geometric considerations, to eliminate all possible off-axis paths. The overall length determines the angular resolution. The first intermediate grid (j) is placed midway between grid (i) and the right end grid; grid (k) is placed midway between grid ( $j$ ) and the right end grid, etc.. The geometry allows a minimization of the number of grids actually needed to achieve the desired overall angular resolution, based on the number of slits in each grid. McGrath ${ }^{[16]}$ has estimated that the best collimation that can be achieved with this device is 30 arc minutes with $40 \%$ transmission.

There are several problems inherent to the above device especially when viewed from the application to hard (over $8 \mathrm{kev}$ ) x-radiation. One would require that each grid be opaque to the radiation of interest because some beams are blocked by only one plate thickness. Also, this apparently simple instrument requires very close alignment tolerances, especially for the closely spaced grids near the beam exit. This makes the device quite difficult to construct. A collimator in which the grids contain a random array of apertures has been suggested ${ }^{[17]}$ and constructed ${ }^{[18]}$, which to some degree eliminates these problems. With an array of identical grids with random apertures, each part of the beam must pass through the same hole of each grid, and the grid plates are evenly spaced. Tests ${ }^{[18]}$ of this device using 8 plates, $1.27 \times 10^{-2} \mathrm{~m}$ 
apart, yielded an angular response at $\mathrm{A} 1 \mathrm{~K} \alpha\left(8.34 \mathrm{~A}^{\circ}\right)$ of 15 arc minutes and $27 \%$ overall transmission. These plates were $25 \times 10^{-6} \mathrm{~m}$ thick stainless steel with 2000 holes, $0.36 \times 10^{-3} \mathrm{~m}$ in diameter, inside $2 \times 10^{-4} \mathrm{~m} 2$. The random hole pattern was derived from a random computer output and photographic etching techniques. A similar output could be used to prepare a tape for a tape operated drilling machine and much thicker plates, suitable to hard $x$-radiation.

An offshoot of this device, the modulation collimator ${ }^{[19]}$, is the current favorite of $x$-ray astronomers. Used in its rotating mode ${ }^{[20] \text {, }}$ it can be used $[21]$ to reconstruct the location of celestial $x$-ray sources down to a few seconds of arc. The multiple set of grids is replaced with multiple grids of wire. The spacing and the wire diameters are determined by the same geometrical arguments as above. The resultant collimator has multiple angular transmission planes. If the modulation collimator is rotated about the line of sight, the $x$-ray signal from each source is modulated with frequency components and with a phase characteristic of each source's location in the field of view. The resultant time varying signal can be Fourier analyzed to reconstruct the source locations. Clearly, both the actual alignment and the precision calibration of a modulation collimator are quite complex. While these developments were underway in mechanical collimation, another very successful means of collimation was also being explored. This method, first suggested by DuMond $[22]$, makes use of multiple parallel reflections by a very good crystal near the Bragg angle to 
achieve highly collimated, very monochromatic x-ray beams. The Bragg condition for one crystal can be represented by a band. After successive diffraction by two or more crystals, a very narrow-range of wavelength and angular divergence, corresponding to the overlapping of the several bands, is selected. With asymmetric diffraction, the very parallel beam can also be magnified in area $[1,4,5,7,23,24]$.

This is the principle used for our asymmetric crystal topography (ACT). The angular collimation from a two crystal arrangement using this principle was found to be much less than 10 arc seconds in the ACT system. Aiso the collimated beam still was sufficiently strong, as demonstrated by the successfully imaged topographs mentioned in Section III. Using a multi-crystal arrangement, the narrowest angular spread actually measured was 0.16 arc seconds, well beyond the usual limits of mechanical collimation. Unfortunately, the collimated beam in this case was quite weak after two or three reflections. Nevertheless, the 10 arc second collimation achieved from two crystal arrangement is much better than that achieved by other techniques.

In sum, it is concluded at the present time that the collimation for the ACT system is the best among those above mentioned collimation methods, particularly because the collimated beam is sufficiently intense (or bright) for practical use。

Resolution With Various Screens

Currently, image intensifiers for visible light are commercially available at reasonable cost. These can be made into $x$-ray image 
intensifiers by placing a fluorescent (phosphor) screen in front of the fiber-optic input faceplate as described in previous papers $[2,7]$. Obviously, the resolution of the $x$-ray image depends strongly on the properties of the phosphors。 We have tested various phosphors, such as polycrystalline cadmium sulfide and gadolinium oxy-sulfide, and single crystal cesium iodide.

For the previous topographic work, a $0.1 \mathrm{~mm}$ thick Ag-activated $(\mathrm{Cd}, \mathrm{Zn}) \mathrm{S}$ fluorescent screen was used. This material was selected simply for its adequate intensity yield of visible (green) light after the conversion from x-rays. Therefore, the resolution was not good for real-time imaging. We tested five additional (Cd, $\mathrm{Zn}$ ) $\mathrm{S}$ phosphors for resolution and intensity-sensitivity (brightness) with CuKa(8kev), MoKa $(17 \mathrm{kev})$ and $\operatorname{AgK\alpha }(22 \mathrm{kev})$ radiation. Nichrome, Sn-bronze, stainless steel and gold meshes of various sizes were placed in the path of a beam collimated by the method described previously. We were able to achieve a resolution of $250 \mu \mathrm{m}$ with all of the phosphors, but their intensitysensitivity varied enormously. As expected, the brightest image was obtained using the coarsest phosphor.

A series of gadolinium oxy-sulfide $\left(\mathrm{Gd}_{2} \mathrm{O}_{2} \mathrm{~S}\right)$ and yttrium oxy-sulfide $\left(\mathrm{T}_{2} \mathrm{O}_{2} \mathrm{~S}\right)$ were tested next. Their particle sizes were quoted to be $6 \mu \mathrm{m}$ and $3 \mu \mathrm{m}$, respectively. The thicknesses of gadolinium phosphors were 17 $\mathrm{mg} / \mathrm{cm}^{2}$ and $19 \mathrm{mg} / \mathrm{cm}^{2}$, and those of yttrium phosphors are all $19 \mathrm{mg} / \mathrm{cm}^{2}$. Their resolution reached $125 \mu \mathrm{m}$, but the brightness from yttrium phosphors was so low that they were considered to be impractical. It seems quite difficult to prepare thin phosphors from fine particles for 
the further improvement of resolution. Therefore, we attempted to prepare phosphors from single crystals.

We chose cesium iodide (C'SI) single crystals for this purpose. For slicing and polishing, we modified the acid saw and the acid polisher which were used for copper and nickel single crystals. Cutting was made using nichrome wires carrying a solution of 5 to $10 \%$ distilled water in ethyl alcohol. The proper speed was chosen by trial and error for each boule of a different diameter. Polishing was tried in two ways:

1) Mechanical polishing - crystal slices were polished first on abrasive papers to obtain a desired thickness and then on a lapping wheel to produce an optical finish on the surfaces。

2) Chemical polishing - slices were polished with 5 to $10 \%$ water in alcohol on a precision polishing wheel attached to the modified acid polisher. Even though considerable efforts were made on the precision for the flatness and the rotation axis of the polishing wheel, this polishing method produced lens shaped samples due to the chemical reaction around the edge of the slice.

It was important to hold slices securely without straining them during polishing. Various methods were tried; for example, "Opticon UV-57" optical cement* was used with only partial success. This produced uneven bonding. Dow Corning "2-3067 optical couplant"* was found to be satisfactory. This is particulary suitable for mounting a crystal on fiber optic plates. The polished crystals were finally mounted on

\footnotetext{
Use of the trade name does not constitute a product endorsement.
} 
fiber optic plates which matched the plate on the front of the image tubes。

In spite of all these efforts, resolution was not improved much from the one $(\sim 125 \mu \mathrm{m})$ obtained with polycrystalline phosphors. The brightness was not improved either. Some of single crystal phosphors yielded less brightness than polycrystalline ones, probably due to the uneven thickness of single crystal wafers. The very thin single crystals showed a marked decrease in brightness. They were impractical for use in the image tubes, even though the resultant resolution was better than that achieved from others。

In conclusion, the present approach in the improvement of resolution for real-time imaging always encounters two opposing problems simultaneously; if one improves the resolution, then the brightness decreases, and vice versa. The best resolution which is easily obtained by the ordinary preparation of phosphors can be said from our experience to be about $150 \mathrm{\mu m}$, without loss of significant brightness. If one uses commercial inexpensive image intensifiers like the one we used, they have a resolution limit determined by the size of the fiber diameter in fiber optical plates. They may be about $50 \mu \mathrm{m}$ down to $10 \mu \mathrm{m}$ at best. Therefore, the present approach or any similar approach would not succeed in reducing the resolution for real-time imaging to less than $10 \mu \mathrm{m}$ or to a submicron range. As a result, we have since taken a completely different approach mostly based on reexamining existing collimation techniques to overcome the resolution problem. This new approach including a two crystal arrangement mentioned above has been quite successful. 


\section{References}

[1] M. Kuriyama, J。R。Early and H. E。 Burdette, NBS report 10873 (1972); NBSIR 73-402, 3 (1973)。

[2] M。 Kuriyama, W。J. Boettinger, H. E. Burdette and R。M. Eaton, NBSIR 74-611, 3 (1974)。

[3] M. Kuriyama, W. J. Boettinger, and H. E. Burdette, NBSIR 76-980, 3, (1976); NBSIR 77-1208, 3 (1977).

[4] M。Kuriyama, J. G. Early and H: E. Burdette, Proc. AIAA 12 th Aerospace Sciences Meeting, Paper No. 74-204 (1974) .

[5] M. Kuriyama, J. G. Early and H. E. Burdette, J.Appl. Cryst. 7, $535(1974)$.

[6] W. J. Boettinger, H. E. Burdette and M. Kuriyama Phil. Mag。 34, $119(1976)$.

[7] W。J。Boettinger, H. E. Burdette, M。Kuriyama and R. E. Green, Jr., Rev. Sci. Instr. 47,18 (1976).

[8] W. J. Boettinger, H。E. Burdette, E. N. Farabaugh and M. Kuriyama, Advances in X-Ray Analysis, Vol。20, Eds. H. E. McMurdie, C. S. Barrett, J。B. Newkirk and C。O。Ruud (1977) p. 207.

[9] W. J. Boettinger, H. E. Burdette and M. Kuriyama, Phi1. Mag. 36, $763(1977)$

[10] M. Kuriyama, W. J. Boettinger and H. E. Burdette, Advances in X-Ray Analysis, Vol. 20, Eds。H。E. McMurdie, C. S. Barrett, J. B. Newkirk and C. O. Ruud (1977) p. 245。

[11] M. Kuriyama, W。 J. Boettinger and H. E. Burdette, J. Cryst. Growth $\underline{43}, 287(1978)$. 
[12] W. Soller, Phys. Rev . 24, 158 (1924).

[13] J. Drahokoupil, Czech. J. Phys. 6, 35 (Jan. 1956).

[14] F. Fournier, Compes Rendus de 1'Acad. Sci. 227, 833 (1948).

[15] J. A. Lely and T. W. van Rijssel, Acta Cryst. 2, 337 (1949) and Philips Tech. Rev. 13, 96 (Oct. 1951).

[16] J. F. McGrath, Jr., Rev. Sci. Inst。39, 1036 (July 1968).

[17] J.H. Underwood, Rev. Sci. Inst. 40, 894 (July 1969).

[18] R。S. Wriston, Rev. Sci. Inst. 45, 1566 (Dec. 1974).

[19] M. Oda, App1. Opt. 4, 143 (1965).

[20] H. W. Schnopper, R。 I. Thompson, and S. Watt, Sp. Sci. Revs. 8 , 534 (1968).

[21] H. Bradt, G. Garmire, M. Oda, G. Spada, B. V. Sreekantaw, P. Gorenstein, and H. Gursky, Sp. Sci. Revs。ㅁ, 471 (1968)。

[22] J.W. M. DuMond, Phys。Rev. 52, 872 (1937).

[23] K. Kohra and S。Kikuta, Acta Cryst. A24, 200 (1968).

[24] M. Kuriyama and W。J. Boettinger, Acta Cryst. A32, 511 (1976). 
Task 4

A Thermochemical Study of Corrosive Reactions in Oxide Materials H. S. Parker, R. S. Roth and C. D. Olson Ceramics, Glass and Solid State Science Division and E. R. Plante

Chemical Stability and Corrosion Division Center for Materials Science

\section{Summary}

The system $\mathrm{KFeO}_{2}-\mathrm{Fe}_{2} \mathrm{O}_{3}$ has been examined over portions of the subsolidus region. The occurrence of a $\beta$-alumina structure-type at a composition $\mathrm{K}_{2} \mathrm{O}: 5.75 \mathrm{Fe}_{2} \mathrm{O}_{3}\left(85.2\right.$ mole percent $\left.\mathrm{Fe}_{2} \mathrm{O}_{3}\right)$ has been observed. Little corrosion of platinum by this composition at a temperature of $1475^{\circ} \mathrm{C}$ was detected. Vapor pressure measurements of $\mathrm{KFeO}_{2}$ showed an equilibrium potassium pressure of the order of $10^{-4}$ atmospheres in the $1200^{\circ} \mathrm{C}$ range. 


\section{Introduction}

The major objective of this work is to further the understanding of the reactions of alkali oxides and iron oxides with other oxides common in MHD and fuel ceil environments. The alkali oxides as a class and the transition elements, with iron oxide as an example, are extremely reactive, particularly in the liquid state. Because of sample-container reaction in laboratory studies, a further objective is to investigate the nature, extent and limitations imposed by this reaction and to evaluate containerless techniques for melt and solid state studies. At the present time, severe problems associated with chemical reaction, corrosion and erosion exist with electrode-insulator materials in the current generation of MHD channels as a result of the $\mathrm{K}_{2} \mathrm{O}$ derived from the required potassium seed together with various combinations of $\mathrm{K}_{2} \mathrm{O}, \mathrm{SiO}_{2}, \mathrm{Fe}_{2} \mathrm{O}_{3}, \mathrm{Al}_{2} \mathrm{O}_{3}$ and other oxides in those MFD channels operated with slagging electrodes (coal combustion). Additionally, one proposed fuel cell concept envisions a molten alkali carbonate eutectic cell with a ceramic matrix, again with a concomitant corrosion problem. However; the study of reactions with alkali oxides, iron oxides and other ceramic oxides is severely hampered and results often vitiated by corrosive reaction with the necessary container material, whether it be ceramic or metal. Thus, it is suggested that the ultimate studies could best be performed in a zero gravity environment where containerless studies might more easily be performed. It is anticipated that such experiments could provide data of importance not 
only to the technological community concerned with alternate energy sources, but in addition provide fundamental data on the basic equilibrium assemblages in such systems. Studies in the $\mathrm{K}_{2} \mathrm{O}-\mathrm{Al}_{2} \mathrm{O}_{3}-$ $\mathrm{Fe}_{2} \mathrm{O}_{3}-\mathrm{SiO}_{2}$ system are currently in progress at NBS as part of our program on materials degradation in an MHD environment. Complementary investigations in a containerless geometry would enable measurements otherwise unobtainable to be made as well as an estimate of the effect of container reaction on properties of importance in MHD applications. As indicated above, the problems of greatest urgency are those involving iron and alkali oxides in contact with ceramic oxides, primarily $\mathrm{Al}_{2} \mathrm{O}_{3}$ and $\mathrm{MgO}$, with the resulting degradation of properties through compound formation or eutectic melting. Attempts to investigate potential solutions to these problems in the laboratory are severely hampered by the simultaneous reduction of iron oxide and alloying with the platinum container and the reaction of the alkali oxides with the platinum container. Both of these reactions severely compromise the bulk composition of the sample under investigation. $\mathrm{Al}_{2} \mathrm{O}_{3}$ and $\mathrm{MgO}$ crucibles have both proven unsatisfactory containment materials, reaction with these oxides elther forming compounds or penetrating the crucible walls with subsequent disruption on cooling. For limited studies, containers of a silver-palladium alloy are of some use at temperatures up to $1200-1400^{\circ} \mathrm{C}$. However, temperatures well in excess of $1400^{\circ} \mathrm{C}$ are of interest in MHD environments. A further problem urgently requiring investigation is associated with the geometry 
and materials in current MHD channels. Iron oxide is one constituent of the electrodes which are in contact with aluminum oxide or MgO insulators. A further complication is associated with the slag which contains potassium, silica, alumina and iron oxide. Reaction of slag with the oxide insulators and electrodes degrades the performance of both and is a major problem at the present time. These reactions include compound formation, melting and vaporization.

The inftial system chosen for investigation is the $\mathrm{KFeO}_{2}-\mathrm{FeO}_{\mathrm{x}}$ portion of the $\mathrm{K}_{2} \mathrm{O}-\mathrm{FeO}_{\mathrm{x}}$ system, in part because of its immediate applicability to the study of slag-electrode reactions in MHD systems. of further interest in this system are those phases with the $\beta$-alumina structures which may exhibit high ionic conductivity as well as high electronic conductivity. During the period covered by this report, the major effort has been directed towards a general survey of the subsolidus phase equilibrium assemblages and vapor pressure measurements of $\mathrm{KFeO}_{2}$.

Experimental Procedures

In order to prevent hydration of both starting materials and heated samples it was necessary to perform all handling operations including weighing, blending and grinding in a drybox. Transfer of materials between laboratories was done in small dessicators. Potassium carbonate and $\alpha-\mathrm{Fe}_{2} \mathrm{O}_{3}$, ignited to constant weight, were used as starting materials. All calcinations were done in air, using gold containers. The heating schedule followed for all 
compositions was an initial heating at $625^{\circ} \mathrm{C}$ for $\sim 18$ hours followed by an $800^{\circ} \mathrm{C}$ heat treatment for $\sim 90$ hours, with periodic grindings. A total of 16 compositions, as shown in Figure 1, were prepared. For heatings above $800^{\circ} \mathrm{C}$, it was necessary to use sealed containers to prevent potassium loss from the specimens. In order to determine if the tubes leaked during heating, specimens were quenched into paraffin oil. Oil was used instead of water to prevent the introduction of moisture into the drybox in case of fallure. Attempts were made to use gold tubes for $1000^{\circ} \mathrm{C}$ heatings but the high failure rate necessitated the use of platinum tubes. At temperatures so close to the gold melting point the gold tubes appeared to fail from internal pressure rather than from any corrosive reaction.

Al1 sixteen compositions were heated at $1000^{\circ} \mathrm{C}$ for 72 hours in sealed platinum tubes, $5 \mathrm{~mm}$ O.D. $\times 25 \mathrm{~mm}$ long, to provide well-reacted materials for higher temperature heatings.

Characterization of specimens after heat treatment was by powder $x$-ray diffraction methods, using a high angle goniometer equipped with a bent graphite crystal monochromator. Scanning speed was $0.25^{\circ} 2 \theta$ per minute. The goniometer shield was sealed and a thin plastic window provided for the $\mathrm{x}$-ray beams. In this manner a dry nitrogen atmosphere could be maintained over the sample, preventing hydration.

Both optical and scanning electron microscopy were used to evaluate the nature and extent of reaction with the platinum container. In this case, a special heavy-walled tube, $2.54 \mathrm{~mm} \mathrm{I.D.} x 6.0 \mathrm{~mm}$ O.D., 
was used. A separate end cap was welded on after filling. After heating, the sample was mounted in epoxy resin and cut with a diamond saw at right angles to the tube axis. The halves were ground and polished using gamma $\mathrm{Al}_{2} \mathrm{O}_{3}$ and methanol for microscopic examination. Experimental Results

A. Phase Equilibria and Corrosion (H.S. Parker, R.S. Roth, C.D. Olson) The compositions and temperatures investigated are shown in Table 1 and Figure 1. Figure 1 includes several high temperature points obtained prior to the start of this investigation. These points were obtained in sealed platinum tubes, with severe attack of the platinum, failure occurring for all compositions within moments when liquid was present. The dashed Iines in Figure 1 are estimates of the solidus and liquidus values primarily from Takahashi, Kuwabara and Kase [1], modified by the NBS measurement of the melting point of $\mathrm{KFeO}_{2}$ as $1615^{\circ} \pm 10^{\circ}$ (C.L. McDaniel, personal communication). The observed phase assemblages are, in general, in agreement with reference 1, although the composition range of $\beta+\beta^{\prime \prime}$ structures appears to be slightly different.

The $\mathrm{K}_{2} \mathrm{O}: 5.75 \mathrm{Fe}_{2} \mathrm{O}_{3}$ composition ( 85.2 mole percent $\mathrm{Fe}_{2} \mathrm{O}_{3}$ ) in the sealed heavy wall platinum tube was heated at $1475^{\circ} \mathrm{C}$ for 19 hours and rapidly cooled in paraffin oil. A low magnification SEM photograph after sectioning, polishing and carbon coating is shown in Figure 2. As is evident, no gross attack of the platinum is visible. As shown in Figure 3a, the specimen was at least partially melted and separated into two phases on cooling. Figures $3 b$ and $3 c$ indicate 
Table 1. The System $\mathrm{KFeO}_{2}-\mathrm{Fe}_{2} \mathrm{O}_{3}$.

\begin{tabular}{|c|c|c|c|c|}
\hline \multicolumn{2}{|c|}{$\frac{\text { Composition }}{\text { Mole percent }}$} & \multirow[t]{2}{*}{$\frac{\text { Temperature }}{{ }^{\circ} \mathrm{C}}$} & \multirow[t]{2}{*}{$\frac{\text { Time }}{\text { hrs }}$} & \multirow[t]{2}{*}{ Results } \\
\hline $\mathrm{K}_{2} \mathrm{O}$ & $\mathrm{Fe}_{2} \mathrm{O}_{3}$ & & & \\
\hline 50 & 50 & 1000 & 72 & $\mathrm{KFeO}_{2}$ \\
\hline 33.33 & 66.67 & 1000 & 72 & $\mathrm{KFeO}_{2}+\left(\beta+\beta^{\prime \prime}\right)$ \\
\hline 25 & 75 & 1000 & 72 & $\mathrm{KFeO}_{2}+\left(\beta+\beta^{\prime \prime}\right)$ \\
\hline \multirow[t]{2}{*}{20} & 80 & 1000 & 72 & $\left(\beta+\beta^{\prime \prime}\right)+\mathrm{KFeO}_{2}$ \\
\hline & & 1200 & 19 & Leaked badly \\
\hline 16.67 & 83.33 & 1000 & 72 & $\left(\beta+\beta^{\prime \prime}\right)+\mathrm{KFeO}_{2}$ \\
\hline 15.4 & 84.6 & 1000 & 72 & $\left(\beta+\beta^{\prime \prime}\right)+$ trace $\mathrm{KFeO}_{2}$ \\
\hline \multirow[t]{4}{*}{14.8} & 85.2 & 1000 & 72 & $\left(\beta+\beta^{\prime \prime}\right)$ \\
\hline & & 1200 & 19 & Single phase $\beta$ \\
\hline & & 1308 & 18 & Single phase $B$ (small leak) \\
\hline & & 1475 & 19 & For SEM examination \\
\hline \multirow[t]{2}{*}{14.3} & 85.7 & 1000 & 72.5 & $\left(\beta+\beta^{\prime \prime}\right)+$ trace $\mathrm{Fe}_{2} \mathrm{O}_{3}$ \\
\hline & & 1308 & 18 & Single phase $\beta$ (small leak) \\
\hline \multirow[t]{2}{*}{12.5} & 87.5 & 1000 & 72 & $\left(\beta+\beta^{\prime \prime}\right)+$ trace $\mathrm{Fe}_{2} \mathrm{O}_{3}$ \\
\hline & & 1200 & 18 & Single phase $\beta$ \\
\hline 11.1 & 88.9 & 1000 & 72 & $\left(\beta+\beta^{\prime \prime}\right)+\mathrm{Fe}_{2} \mathrm{O}_{3}$ \\
\hline \multirow[t]{2}{*}{10.0} & 90.0 & 1000 & 72 & $\mathrm{Fe}_{2} \mathrm{O}_{3}+\left(\beta+\beta^{\prime \prime}\right)$ \\
\hline & & 1200 & 18 & Leaked badly \\
\hline 9.1 & 90.9 & 1000 & 72 & $\mathrm{Fe}_{2} \mathrm{O}_{3}+\left(\beta+\beta^{\prime \prime}\right)$ \\
\hline 8.3 & 91.7 & 1000 & 72 & $\mathrm{Fe}_{2} \mathrm{O}_{3}+\left(\beta+\beta^{\prime \prime}\right)$ \\
\hline 7.7 & 92.3 & 1000 & 72 & $\mathrm{Fe}_{2} \mathrm{O}_{3}+\left(\beta+\beta^{\prime \prime}\right)$ \\
\hline 5.9 & 94.1 & 1000 & 72 & $\mathrm{Fe}_{2} \mathrm{O}_{3}+\left(\beta+\beta^{\prime \prime}\right)$ \\
\hline 4.2 & 95.8 & 1000 & 72 & $\mathrm{Fe}_{2} \mathrm{O}_{3}+\left(\beta+\beta^{\prime \prime}\right)$ \\
\hline 0 & 100 & 1000 & 72 & $\mathrm{Fe}_{2} \mathrm{O}_{3}$ \\
\hline
\end{tabular}

al All compositions calcined at $625^{\circ} \mathrm{C}$ for $\sim 18$ hours and $800^{\circ} \mathrm{C}$ for $\sim 90$ hours. All heatings above $1000^{\circ} \mathrm{C}$ used material heated at $1000^{\circ} \mathrm{C}$ for 72 hours. 
the lamellae to be a potassium-rich phase. Again, little or no interdiffusion between $\mathrm{Fe}, \mathrm{K}$ and the platinum container is evident.

In Figure 4a, a dark area is visible at the interface between the platinum and specimen near the upper center of the figure. Analysis of this area, Figure 4b, by EDXA showed that a slight reaction has occurred, the dark area being composed of $\mathrm{K}, \mathrm{Fe}$ and $\mathrm{Pt}$ as contrasted to the spectrum obtained in areas immediately adjacent on either side, Pt in one case and $\mathrm{K}$ plus $\mathrm{Fe}$ only in the other.

\section{B. Vapor Pressure Measurements on "KFeO 2 " (E.R. Plante)}

Results of mass spectrometric measurements of the vapor pressure of $\mathrm{KFeO}_{2}$ (series 1 ) were summarized in a previous report [2]. In the series 1 data an abnormally high $I_{32}^{+} / I_{39}^{+}\left(0_{2}^{+} / \mathrm{K}^{+}\right)$ratio was observed which was thought to indicate that significant reduction of the $\mathrm{KFeO}_{2}$ took place during the vaporization experiments. However, this observation was contrary to results obtained by $x$-ray diffraction studies of the residual sample which indicated that the evaporation process was consistent with a reaction forming a non-stoichiometric phase which can be approximated by the following:

$$
7 / 6\left(\mathrm{~K}_{2} \mathrm{O} \cdot \mathrm{Fe}_{2} \mathrm{O}_{3}\right)=2 \mathrm{~K}(\mathrm{~g})+1 / 2 \mathrm{O}_{2}(\mathrm{~g})+1 / 6\left(\mathrm{~K}_{2} \mathrm{O} \cdot 7 \mathrm{Fe}_{2} \mathrm{O}_{3}\right)
$$

The possibility that a high $\mathrm{O}_{2}$ pressure was due to reaction of $\mathrm{FeO}_{\mathrm{x}}$ with Pt allowing release of $\mathrm{O}_{2}$ was considered but SEM examination of the Pt Knudsen cell indicated only minor interaction. 
Because of these conflicting observations the mass spectrometer constant could only be determined within a factor of 3 . If it was assumed that the $\mathrm{x}$-ray diffraction data were correct the mass spectrometer calibration factor for $\mathrm{K}$ would be a factor of 3 greater than that obtanined assuming the $\mathrm{I}_{32}^{+} / \mathrm{I}_{39}^{+}$ratio was correct.

More recent observations on unrelated $\mathrm{K}_{2} \mathrm{O}$-containing slag samples have shown that the $\mathrm{I}_{32}^{+} / \mathrm{I}_{39}^{+}$data were probably incorrect because of misalignment of the molecular beam with the ion source. These observations showed that the $\mathrm{I}_{32}^{+} / \mathrm{I}_{39}^{+}$ratio could be varied over a factor 3 depending on the ion optics adjustments of the source. This effect is possible because $\mathrm{O}_{2}$ is non-condensible and can bounce off surfaces into the active volume of the ion source while $\mathrm{K}$ is condensible and will be effectively removed from the molecular beam.

New measurements on the vaporization of $\mathrm{KFeO}_{2}$ have been undertaken. The measurements were made in our modulated beam, quadrupole mass spectrometer using a platinum effusion cell having a $0.343 \mathrm{~mm}$ diameter orifice. The experimental arrangement was essentially the same as previously used except that a new thermocouple was used. As before, measurements of both the $\mathrm{K}^{+}$and $\mathrm{O}_{2}^{+}$ion currents were made as a check on the vaporization stoichiometry. However, within experimental error the vaporization reaction is that previously noted.

Certain non-equilibrium effects were observed during the experiments. Measurements of the ion currents were made at constant temperature, but the initial ratio of $\mathrm{I}_{32}^{+} / \mathrm{I}_{39}^{+}$depended on whether the temperature was 
increased or decreased in going from one constant temperature to another. When the temperature was increased, after the initial increase in the $\mathrm{K}^{+}$intensity, there was a tendency for the $\mathrm{K}^{+}$ion current to decrease with time and the $0_{2}^{+}$ion intensity to Increase with time until a steady state was reached. The opposite effects were noted when the temperature was decreased but the same steady state was eventually achieved. It appeared, however, that a shorter period of time was required to achieve steady state pressures if the temperature was increased from point to point and the data presented was taken in that direction. Above $1300 \mathrm{~K}$, equilibration rates were quite rapid and steady state pressures were observed within 10-15 minutes, while at the lower temperatures periods of about an hour were required.

These effects could be due to a phase boundary dependence on temperature. For example, the " $\mathrm{K}_{2} \mathrm{O} \cdot 7 \mathrm{Fe}_{2} \mathrm{O}_{3}$ " composition might become $\mathrm{O}_{2}$ deficient at higher temperatures, Other possibilities include instrumental problems with the mass spectrometer or kinetic factors in the diffusion of $\mathrm{K}$ and $\mathrm{O}_{2}$ from the evaporating phase (insufficient equilibration time). At steady state conditions, the $I_{32}^{+} / I_{39}^{+}$ratio indicated a deficiency of $\mathrm{O}_{2}$ at low temperatures but at higher temperatures the $\mathrm{O}_{2} / \mathrm{K}$ pressure ratio appeared consistent with the proposed decomposition reaction.

Some selected data from the measurements are shown in Figure 5. Curve A shows data for the $K$. pressure series $I$ data using the mass 
spectrometer sensitivity constant obtained by assuming that decomposition took place according to the reaction cited. Curve $\mathrm{B}$ is the $\mathrm{K}$ pressure observed during the later experiments and Curve $\mathrm{C}$ is the oxygen pressure. The ratio of the $\mathrm{K}$ pressure to the $\mathrm{O}_{2}$ pressure is based on previous experience which indicates that the mass spectrometer sensitivity constant for $K$ is about 5.6 times that for $0_{2}$. The difference between curves $A$ and $B$ is probably due to a systematic error in temperature of about $30 \mathrm{~K}$. Different thermocouple probes were used for the respective measurements. The curve $B$ data is thought to be more reliable. Curve $\mathrm{D}$ represents the $\mathrm{K}(\mathrm{g})$ pressure over $\mathrm{KAlO}_{2}$. The order of magnitude increase in the $\mathrm{K}$ pressure over $\mathrm{KFeO}_{2}$ compared to $\mathrm{KAlO}_{2}$ confins the general idea that $\mathrm{K}_{2} \mathrm{O}$ interacts more strongly with $\mathrm{Al}_{2} \mathrm{O}_{3}$ than with $\mathrm{Fe}_{2} \mathrm{O}_{3}$.

Conclusion and Discussion

The compositional range of the $\beta-\beta$ " alumina structure types appears to be centered at a composition of $\mathrm{K}_{2} \mathrm{O}: 5.75 \mathrm{Fe}_{2} \mathrm{O}_{3}$ (85.2 mole percent $\mathrm{Fe}_{2} \mathrm{O}_{3}$ ) with the $\beta^{\prime \prime}$ disappearing at higher temperatures as the $\mathrm{Fe}^{+2}: \mathrm{Fe}^{+3}$ ratio increases. The possibility of high ionic and electronic conductivity in these structures, including the $\beta^{\prime \prime \prime}$ structure should be investigated.

The attack of platinum by compositions in the 85 mole percent $\mathrm{Fe}_{2} \mathrm{O}_{3}$ region does not appear to be extensive at temperatures up to $1475^{\circ} \mathrm{C}$ for times of 19 hours. Failure of thin-wall platinum containers at temperatures in the $1200^{\circ}-1300^{\circ} \mathrm{C}$ range may be due to internal 
pressure from released oxygen from the sample rather than from corrosive effects. Future Mossbauer and magnetic susceptibility studies should help establish the cause of failure. At compositions higher in potassium content, it is expected that corrosive attack will become a factor.

The potassium pressure over $\mathrm{KFeO}_{2}$ is roughly an order of magnitude greater than that over $\mathrm{KAIO}_{2}$, approaching $10^{-4}$ atmospheres in the $1200^{\circ} \mathrm{C}$ range. As a result, shifts in bulk composition occurring during extended heat treatments at higher temperatures in open systems must not be overlooked.

\section{References}

[1] T. Takahashi, $\mathrm{K}$. Kuwabara, and $\mathrm{Y}$. Kase, "Formation of $\mathrm{K}-\mathrm{BFe}_{2} \mathrm{O}_{3}$ and Phase Diagram of the System $\mathrm{KFeO}_{2}-\mathrm{Fe}_{2} \mathrm{O}_{3}$ ", Denk1 Kagaku 43 [5] 273-7 (1975). In Japanese.

[2] Quarterly Progress Report for July - September 1977, NBS

"Properties of Electronic Materials", E. Passaglia, NASA Government Order H-27954B, October 1977. 


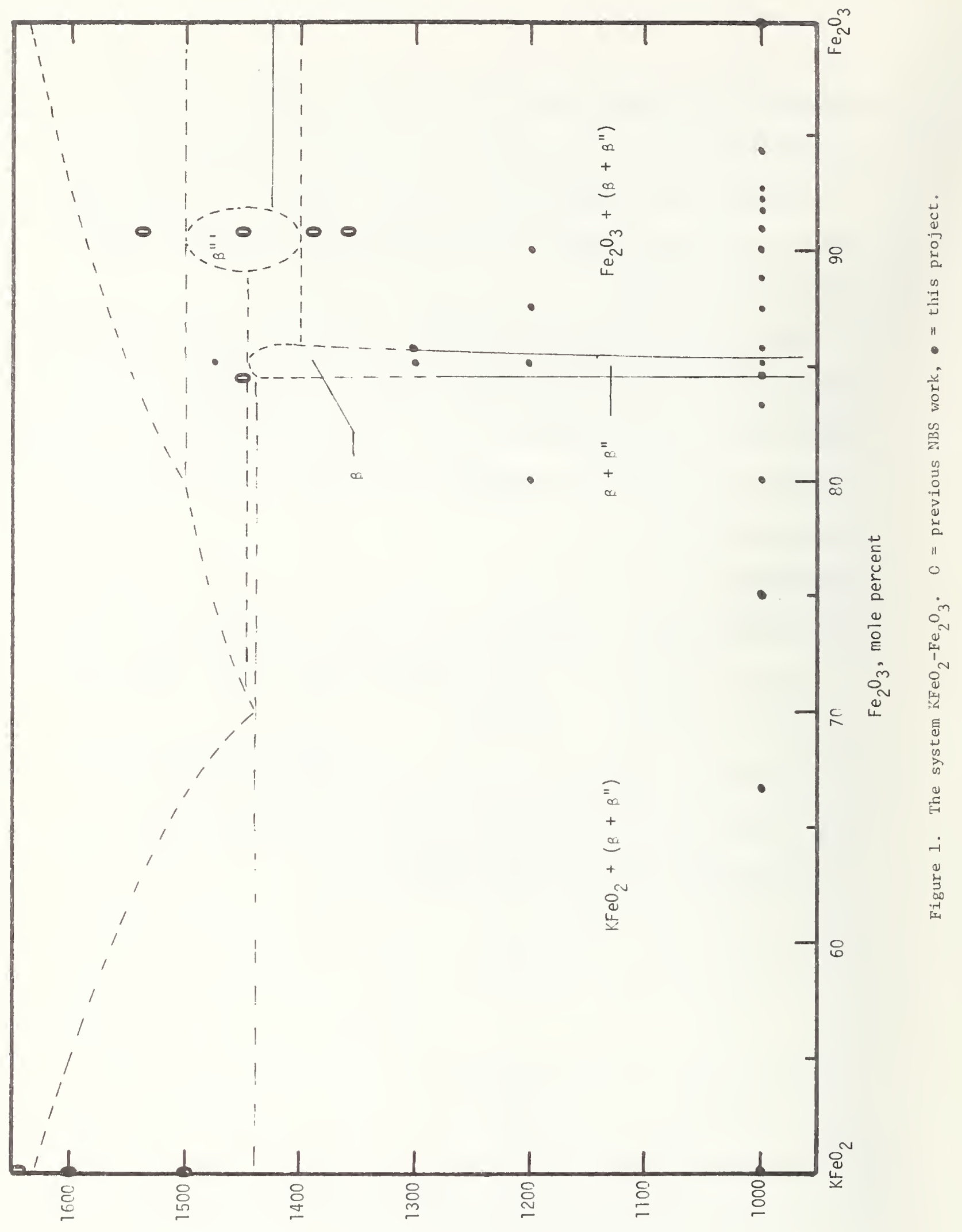




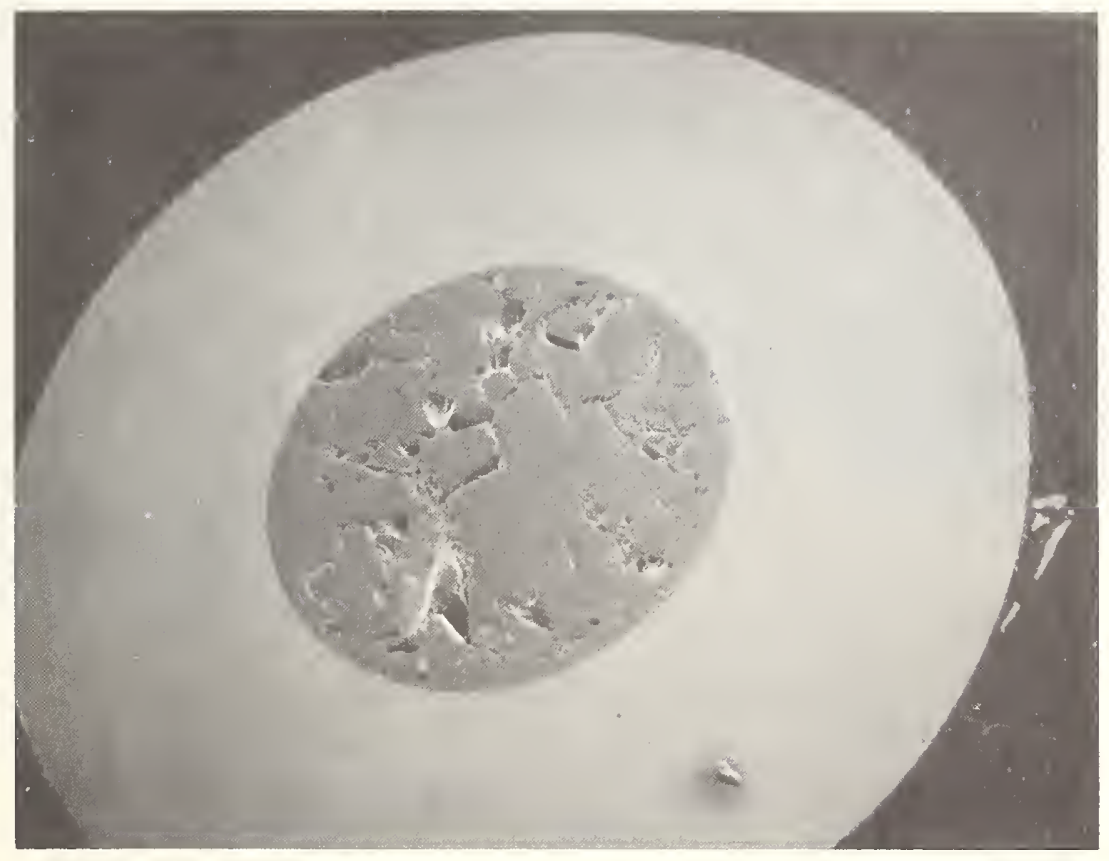

$1 \mathrm{~mm}$

Figure 2. Scanning electron micrograph of section of heavy wall platinum tube contalning specimen of $\mathrm{K}_{2} \mathrm{O}: 5.75 \mathrm{Fe}_{2} \mathrm{O}_{3}$ (85.2 mole percent $\mathrm{Fe}_{2} \mathrm{O}_{3}$ ) after heat treatment at ${ }^{3}$ $1475^{\circ} \mathrm{C}$ for 19 hours. 


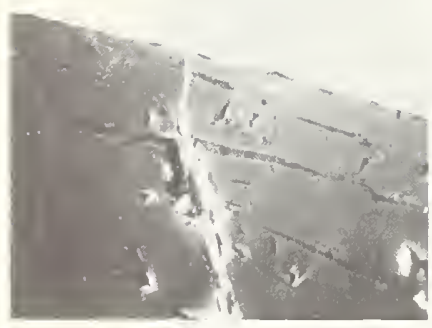

(a)

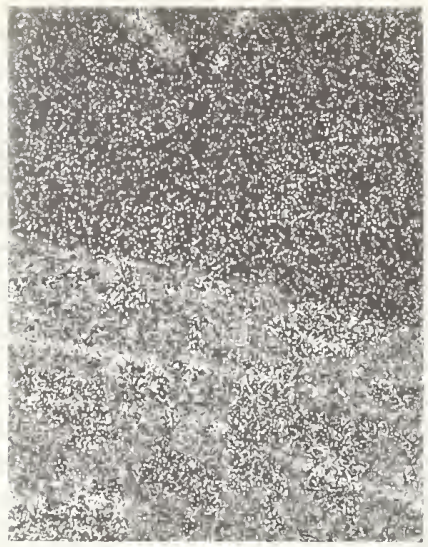

(b)

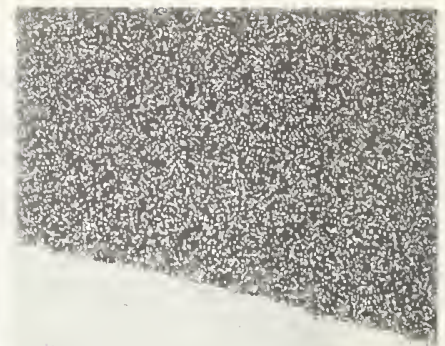

$x$

(c)

$0.02 \mathrm{~mm}$

Figure 3. a) Scanning electron micrograph of interface of specimen of Figure 2 .

b) Potassium map of $3(a)$.

c) Iron map of $3($ a). 


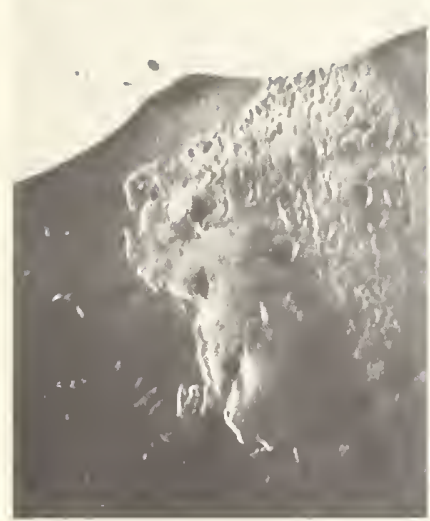

(a)

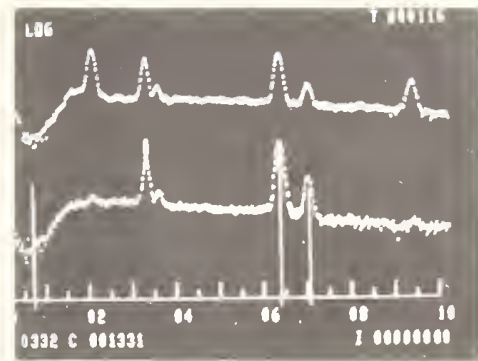

(b)

$0.01 \mathrm{~mm}$

Figure 4. a) High magnification micrograph of platinum-sample interface of specimen of Figure 2 .

b) EDXA spectrum of small dark area near upper center of $4 \mathrm{a}$ and spectrum of immediately adjacent specimen. 


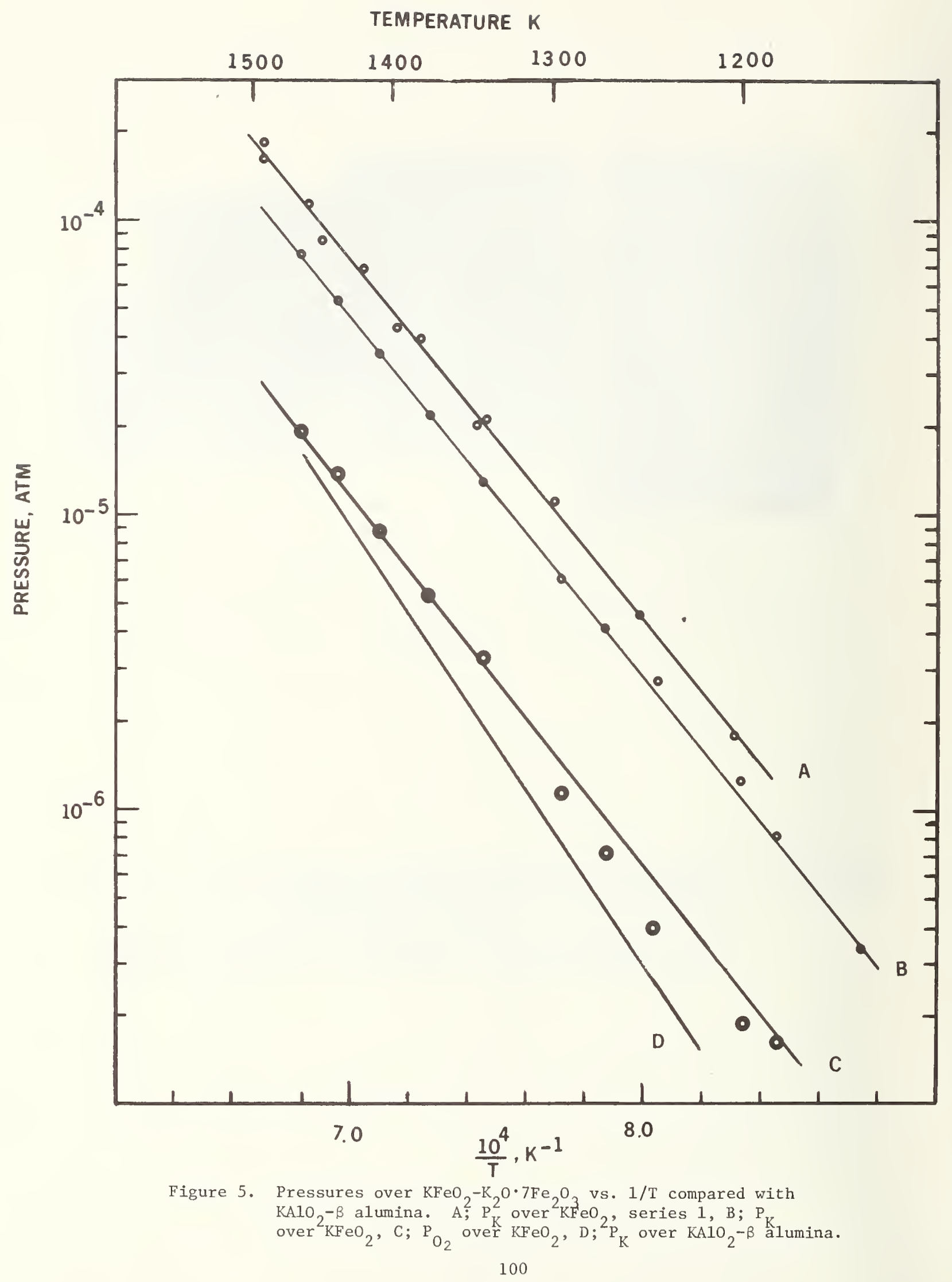




\author{
Task 5 \\ Gravity Effects on Flame Inhibition \\ J. W. Hastie \\ Chemica1 Stability and Corrosion Division \\ Center for Materials Science \\ Summary
}

The first year of this project has emphasized the development of new or improved techniques for the detailed molecular characterization of laboratory flames. This development has progressed very satisfactorily, with the following main achievements:

a. Design and nearly complete construction of a High Pressure Sampling Mass Spectrometer facility, suitable for transient species analysis in diffusion and particulate containing flames.

b. Development of optical absorption spectroscopy for $\mathrm{OH}$ concentration and rotational temperature measurements in optically thick flames.

c. Application of the new technique of rotationa1 Raman spectroscopy to flame temperature profile measurements.

d. Initiation of thermodynamic and chemical kinetic modeling predictions of flame temperatures, post flame species concentrations, and flame inhibition and promotion.

e. Design, construction and critical evaluation of new burner systems having potential.utility as standard devices for interlaboratory comparison of flame diagnostic procedures. 
f. Critical evaluation of various optically based flame temperature measurement techniques including, OH-absorption, $\mathrm{N}_{2}$-rotational Laser Raman, NaD-line reversal methods.

\section{Introduction}

Previous experimental and modeling studies of gravity effects in clean or non-inhibited combustion by NASA and NASA-supported research laboratories have considered the fluid-dynamic and thermodynamic aspects and obtained partial agreement between experiment and theory. A significant conclusion from this early work is the recognition that a more satisfactory agreement between observed and predicted gravity-effects cannot be expected until more detailed combustion kinetics are included in the models--even for clean unperturbed flames (e.g., see NASA CR120921, 1972). Such studies have however established that, for gaseous flame mixtures, readily observable phenomena such as physical shape and combustion efficiency are known (NASA CR-120921) and can be predicted (NASA CR-143350) to be strongly modified under zero-gravity conditions. Similarly synthetic polymers, such as polyesters and polyurethanes, exhibit marked differences in flame propagation rate at zero-gravity as compared with normal $1-\mathrm{g}$ conditions (NASA Conf. Mater. Impr. Fire Saf., 1970). As these and related flame properties are also known to be affected by flame inhibitors under normal g-conditions, we can therefore expect the degree and perhaps the mechanism of flame inhibition to be gravity dependent. This means that the control of fires in space by flame inhibitors should not necessarily be dictated by terrestrial experience. Nor should existing standards and test procedures be assumed 
to be valid measures of fire hazards in space. Another aspect to the problem is that the eventual study of laboratory flames in space under essentially zero-gravity conditions would provide us with an added variable (i.e., g-level) for testing and mechanistic details of flame propagation and inhibition in general. This is significant since our present understanding of flame phenomena is limited by the difficulty of uncoupling the physical (e.g., mass and heat transfer) and chemical (e.g., reaction rates) components in flame theory. As gravity can be expected to influence primarily the physical transport properties, a comparison of earth-bound and space-laboratory flame data should reveal the relative importance of physical and chemical effects and aid in their decoupling for development of a generalized flame theory. Thus the study of flames in space impacts both on the reduction of spacecraft fire hazards and in the fundamental understanding of flames under any gravity condition. In the latter instance, the development of a generalized flame theory incorporating chemical or physical perturbations leading to inhibition or promotion will certainly advance the flame retardant and energy conservation technologies. Development of such a far reaching theory will require the most detailed experimental measurement effort possible, so that non-unique mechanisms are excluded as far as possible.

\section{Experimental Procedures}

A comparison of flame inhibition processes under both 1 -g and zerog conditions will be made to test gravity effects on flame inhibition, leading to a generalized model of flame inhibition. From practical and 
fundamental (i.e., availability of basic chemical kinetic data) considerations, a halocarbon such as the commercial fire extinguishing agent $\mathrm{CF}_{3} \mathrm{Br}$ in diffusion and premixed flame configurations, will be required under normal $1-g$ conditions to establish a predictive preliminary model for flame inhibition in a spacecraft environment. The limited availability of basic chemical kinetic data limits the flame compositions for which a theoretical analysis is presently possible to those containing $\mathrm{H}_{2}$ or $\mathrm{CH}_{4}$ as fuel and $\mathrm{O}_{2}$ as oxidant. These flame mixtures will be subjected to detailed earth-lab mass spectrometric and optical spectroscopic analyses for the spatial determination of species concentration and temperature profiles, including both radical intermediates and stable reactant and product species. These profiles are the net result of chemical kinetic and transport processes, and, using the well known flame equations, such data can be unfolded to yield a set of reactions describing flame propagation and inhibition. Thus the profile data will be used to establish a flame inhibition model for $1-g$ conditions. This predictive model will then be used to define the experimental parameters, such as flame composition and flame type (diffusion or premixed), best suited to zero-g conditions. Such an a priori optimization of experimental conditions for a spacecraft study of flame inhibition is essential, due to the limited measurement tools that can be applied in space. Thus the use of a sophisticated molecular-beam-sampling mass spectrometer, for instance, in the terrestrial experiments would be contrasted in suggested space lab studies by application of relatively simple optical spectroscopic methods for monitoring macroscopic flame properties such as luminosity, 
and the spatial distribution of $\mathrm{CO}_{2}$ and $\mathrm{CO}$ species. Eventual comparison of these sky lab data with those obtained in the $1-\mathrm{g}$ environment would be used to test, and modify where necessary, the predictive flame inhibition mode1.

\section{Experimenta1 Results}

The main objectives for the first year of this project (just completed) were as follows: (a) Using molecular beam mass spectrometry obtain temperature and species concentration profiles including radical intermediates for premixed $\mathrm{CH}_{4}, \mathrm{H}_{2}, \mathrm{O}_{2}, \mathrm{~N}_{2}$ flames at atmospheric pressure. (b) Develop optical spectroscopic absorption methods to supplement mass spectrometric technique. (c) Design and construct a separate molecular beam sampling mass spectrometer system for species characterization of relatively weak premixed and diffusion flames. Objectives (b) and (c) were essentially met and objective (a) was modified to include the application of Laser Raman spectroscopy to the monitoring of temperature and species concentration profiles.

Optica1 Spectroscopy:

Classical optical spectroscopic methods have been utilized in our laboratory primarily for spatially resolved concentration measurements of $\mathrm{H}$ and $\mathrm{OH}$ and the temperature profile determinations. Current work has emphasized optical absorption spectroscopic measurements of the $\mathrm{OH}$ visible-U.V. system in a series of fuel-rich $\mathrm{H}_{2}-\mathrm{O}_{2}-\mathrm{N}_{2}$ flames. Particular attention was given to the rotational line intensities and their dependence on the degree of optical thickness of the flame. The basic expressions for deriving temperatures and $\mathrm{OH}$ concentrations from the $\mathrm{OH}$ rotational 
fine structure are valid only for an optically thin system such as for very weak (and difficult to measure)-transitions and for flames with relatively low $\mathrm{OH}$ concentration. By noting the dependence of absorption intensity as a function of the number of light beam traversals (i.e., optical path length) through the flame we have been able to correct for the effect of optical thickness. The multipass optical system used was based on the White cell design. A linear dependence of the fractional absorption on the number of beam traversals was found thus enabling an accurate extrapolation to zero passes to be made from which line intensitites of the ideal optically thin condition are obtained.

With this correction procedure, we have determined average postflame temperatures and $\mathrm{OH}$ concentrations for several $\mathrm{H}_{2}-\mathrm{O}_{2}-\mathrm{N}_{2}$ flame compositions. The importance of the optical thickness correction was evident from the approximately $200 \mathrm{~K}$ temperature difference between the corrected and uncorrected values (too high). Comparison of the corrected temperatures with those obtained by the Na-D line reversal technique indicated a disparity of 100-200K. At least part of this disparity may be due to the lower spatial resolution of the White cell optical system. Laser Raman Spectroscopy:

Laser Raman spectroscopy has the particular advantage over other optical methods of high spatial resolution and sensitivity to major species not having readily accessible electronic transitions. Our current interest in this technique is based on its potential for accurate temperature profile determinations throughout the whole flame. This contrasts with other optical methods, such as the $\mathrm{Na}-\mathrm{D}$ line reversal 
approach, where moderate resolution data can be obtained only in the post flame region.

Vibrational and rotational Raman measurements have been carried out on small premixed $\mathrm{H}_{2} / \mathrm{O}_{2} / \mathrm{N}_{2}$ flames premixed initial compositions of $4 / 1 / 4$ and 3.4/1.0/2.0. Measurements of temperature profiles in the fuel rich 4/1/4 flame with and without a concentric $\mathrm{N}_{2}$ gas shield flow indicates that diffusion of air into the unshielded flame can raise the flame temperature by $125 \mathrm{~K}$ (21.50K unshielded, 2025K shielded at $5 \mathrm{~cm}$ distance from the burner face). Comparison of Na-D line reversal temperature data from the capillary type burner (used for the other optical measurements) and Raman measurements from the shielded torch flames gives temperatures in agreement within the uncertainties of the two measurements ( 20K for Raman).

Computer profile calculations of $\mathrm{N}_{2}$ and $\mathrm{H}_{2}$ vibrational $\mathrm{Q}$ branch lines have been obtained as a function of temperature. These calculations are essentially the same as those used by others (Lapp, Setchell, etc.). The effects of rotational-vibrational line strength corrections, 0 and $S$ branch contributions, and the anisotropic component of the Q branch on these predicted profiles (and hence, temperature measurements) are currently being investigated. For example, including rotationalvibrational line strength corrections for $\mathrm{H}_{2}-\mathrm{Q}$ branch spectra lowers the calculated temperatures by only $1.5 \mathrm{~K}$ at $325 \mathrm{~K}$ but more significantly by $80 \mathrm{~K}$ at $2400 \mathrm{~K}$. 


\section{Mass Spectroscopy:}

The mass spectroscopic component of our flame characterization program relates to the spatially resolved identification of virtually all the molecular species present. As the method involves use of an intrusive molecular beam sampling probe, care must be taken to avoid perturbing the system being sampled. Previous work in our laboratory has demonstrated the conditions for which such a perturbation is negligible. A second high pressure sampling mass spectrometer system (HPMS) is currently under construction in our laboratory and is near completion. This system will be more versatile than our existing HPMS facility in that it will allow sampling from vertical gas flows (i.e., flames) and from diffusion and particulate laden flames.

Associated with both the mass and optical spectroscopic phases of our work is the development of reproducible flat flame burners capable of handling inorganic additivies. We have obtained commercially a metal honeycomb material from which we fabricated a shielded flat flame burner. Unlike the more conventional sintered brass burner-type this burner produced visually flat flames of $\mathrm{CH}_{4}-\mathrm{O}_{2}-\mathrm{N}_{2}$ over a wide range of fuel rich and lean stoichiometries. This burner so far appears very satisfactory for generating atmospheric pressure flat flames suitable for both optical and mass spectrometric analysis as well as eventual theoretical mode1ing. Mode1ing:

With the increasing availability of high speed computer facilities and the development of new mathematical techniques for solving the basic 
flame equations, theoretical modeling of kinetically complex experimental systems is becoming feasible. The potential value of modeling combined with a comprehensive combustion diagnostics program is now well recognized by researchers in the field. While at the current level of effort we cannot generate original modeling computer programs ourselves, we are making every effort to obtain usable programs from other sources as they become available. In the interim we are, and shall continue, modeling selected aspects of the experimental systems.

For instance, the burnt gas region of the hot $\mathrm{H}_{2}-\mathrm{O}_{2}-\mathrm{N}_{2}$ flames that we have obtained experimental data on can be very well approximated as a thermodynamic equilibrium system. The Gordon and McBride NASA multicomponent equilibrium program has been revived for this purpose. Calculations have been made of the adiabatic flame temperatures of methane and propaneair flames of varying stoichiometry in an attempt to rationalize the different behavior of inhibitors in both these types of flames. The calculated flame temperatures differ by less than 50K, with propane yielding hotter flames. This trend is in accord with the observed effect of inhibitors in diffusion flames where smoke suppression effects appear more predominantly for the hotter propane-air flames.

In an attempt to rationalize, in at least a semi-quantitative manner, the experimentally observed reversible inhibition-promotion effect of phosphorus-containing additives we have developed a simple kinetic argument based on the rate limiting reaction;

$$
\mathrm{H}_{2} \mathrm{O}+\mathrm{PO} \rightleftharpoons \mathrm{HPO}_{2}+\mathrm{H} \text {. }
$$


Our previous determination of the heat of formation for $\mathrm{HPO}_{2}$, using Knudsen effusion mass spectrometry, combined with the known thermodynamic data for $\mathrm{PO}, \mathrm{H}_{2} \mathrm{O}$ and $\mathrm{H}$ indicate this reaction to be exothermic by about $22 \mathrm{kcal} / \mathrm{mo} 1$. To a satisfactory approximation we can therefore assume the activation energy for the reverse process to have a similar magnitude. The pre-exponential factor in the Arhenius rate expression can be estimated by analogy with similar processes, e.g., those reported by Jones and Jensen (1975). With this information we have estimated rate constants for the forward and reverse processes as a function of temperature. From our mass and optical spectroscopic analyses of flames containing phosphorus, we know the concentrations of the species involved in the above rate processes. Hence, the reaction rates can be calculated. We find that the calculated reaction rate vs temperature curves for the forward and reverse processes should cross in the region of $1700 \pm 300 \mathrm{~K}$ with the forward process being favored above the cross over temperature. This forward reaction creates $H$ atoms and is therefore $f l a m e$ promoting whereas the reverse process is flame inhibiting. Our earlier reported experimental observations of phosphorus-induced H-atom removal below about $2000 \pm 200 \mathrm{~K}$ and $\mathrm{H}$ atom production above this temperature regime can, therefore, be rationalized by this simple kinetic scheme involving the $\mathrm{PO}$ and $\mathrm{HPO}_{2}$ species.

Conclusion and Discussion

The first year of this project has emphasized the development of new or improved techniques for the detailed molecular characterization of laboratory flames. This development has progressed very satisfactorily, 
with the following main achievements:

a. Design and nearly complete construction of a High Pressure Sampling Mass Spectrometer facility, suitable for transient species analysis in diffusion and particulate containing flames.

b. Development of optical absorption spectroscopy for $\mathrm{OH}$ concentration and rotational temperature measurements in optically thick flames.

c. Application of the new technique of rotational Raman spectroscopy to flame temperature profile measurements.

d. Initiation of thermodynamic and chemical kinetic modeling predictions of flame temperatures, post flame species concentrations, and flame inhibition and promotion.

e. Design, construction and critical evaluation of new burner systems having potential utility as standard devices for interlaboratory comparison of flame diagnostic procedures.

f. Critical evaluation of various optically based flame temperature measurement techniques including, OH-absorption, $\mathrm{N}_{2}$-rotational Laser Raman, NaD-line reversal methods. 
National Aeronautics and Space Administration Washington, D. C. 20546

Mr。F。L。Williams

Dr。J。R。Carruthers
Code ES

Code ES
1 copy

4 copies

National Aeronautics and Space Administration Johnson Space Center

Houston, Texas 77058

Mr。W. E. Rice

Mr。J.P. Loftus

Mr。J.A. Mason

Mr. E。 J。Svreek
Code EA

Code AT

Code DA

Code FM5
2 copies

1 copy

1 copy

1 copy

National Aeronautics and Space Administration George C. Marshall Space Flight Center Marshall Space Flight Center, Alabama 35812

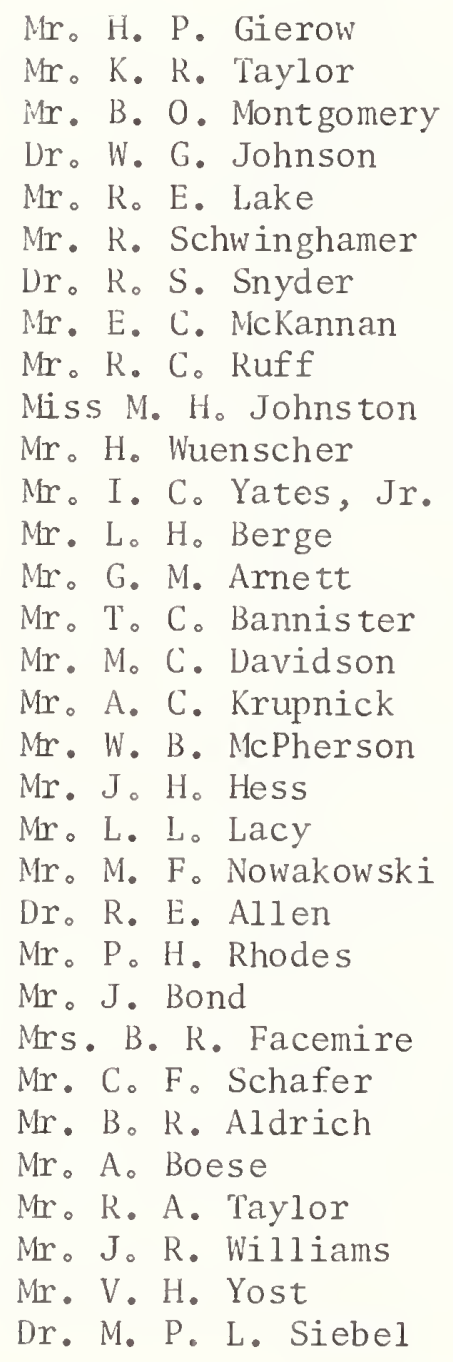

Code PD-MP-DIR

Code PD-MP-T

Code S\&E-DIR

Code S\&E-R-DIR

Code S\&E-R

Code S\&E-ASTN-M

Code S\&E-ASTN-MTE

Code S\&E-ASTN-MM

Code S\&E-ASTN-MEV

Code S\&E-ASTN-MEV

Code S\&्षE-PE-DIR

Code SEEPE-A

Code S\&E-PE-A

Code S\&E-SSL-TR

Code S\&E-SSL-T

Code S\&E-SSL-TR

Code S\&E-ASTN-MT

Code S\&E-ASTN-MMM

Code S\&E-ASTN-MM

Code S\&E-SSL-NP

Code S\&E-QUAL-QT

Code S\&E-ASTN-MTM

Code S\&E-ASTN-MTE

Code SEE-ASTN-MTE

Code SEE-SSL-TR

Code S\&E-SSL-TR

Code S\&E-PE-MXX

Code S\&E-PE-A

Code SE్E-PE-MEI

Code S\&E-PE-M

Code SEE-PE-MW

Code S\&E-PE-DIR
1 copy

2 copies

1 copy

1 copy

1 copy

1 copy

1 copy

1 copy

1 copy

1 copy

1 copy

1 copy

1 copy

1 copy

1 copy

1 copy

1 copy

1 copy

1 copy

1 copy

1 copy

1 copy

1 copy

1 copy

1 copy

1 copy

1 copy

1 copy

1 copy

1 copy

1 copy

1 copy 
Jet Propulsion Laboratory

California Institute of Technology

4800 Oak Grove Drive

Pasadena, California 91103

Dr. C。H. Savage

Dr。M.M. Saffren

Dr。T. G。Wang

Dr. D. D. E1leman

Dr. J. W. Lucas
Code 158-235

Code 183-301

Code 183-401

Code 183-401

Code $180-700$
1 copy

1 copy

1 copy

1 copy

1 copy

National Aeronautics and Space Administration Langley Research Center

Hampton, Virginia 23665

Dr. L。T. Melfi, Jr.

$\mathrm{Mr}$. B. W. Cocke, Jr.

Dr。R。A. Outlaw

Dr. J. P. Mugler

Mr. J。D。DiBattista

Mr. W. C. Ayers
Code 401A

Code 401A

Code 234

Code 215B

Code 215B

Code 418
1 copy

1 copy

1 copy

1 copy

1 copy

1 copy

National Aeronautics and Space Administration Ames Research Center

Moffett Field, California 91103

Dr。J.A. Parker

Code SC

1 copy

Grumman Aerospace Corporation

Bethpage, New York 11714

Dr。C。H. Li

1 copy

General Electric Company

Space Sciences Laboratory

$\mathrm{P}$ 。O 。Box 8555

Philadelphia, Pennsylvania 19101

Dr. R。 T。 Frost

1 copy

European Space Research Organization

114 Avenue Charles de Gaulle

92 Neuilly, France

Dr . G. Seibert

1 copy

Gesellschaft fur Weltraumforschung $\mathrm{mbH}$

505 Porz-Wahn

Lind er Hohe

German Federal Republic

Dr. A. Bewersdorff

1 copy 

NBS.114A (REV. 7.73)

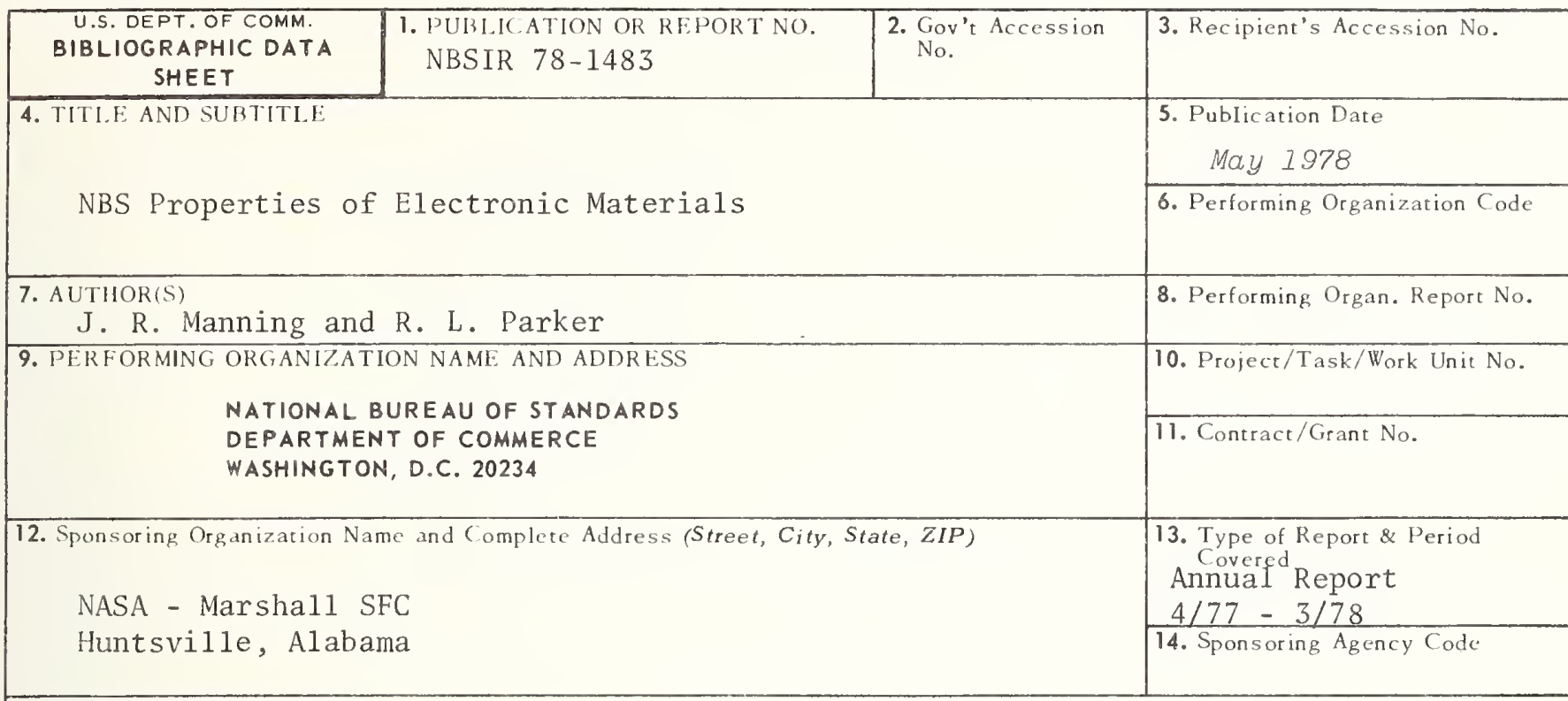

\section{SUPPIEMENTARY NOTES}

Annual Report

16. ABSTRACT (A 200-word or less factual summary of most significant in formation. If document includes a significant bibliography or literature survey, mention it here.)

This report describes NBS work for NASA in support of NASA's (Materials Processing in Space) Program covering the period April 1, 1977 to March 31, 1978.

The resuits obtained for each task are given in detailed summaries in the body of the report. Briefly, in Task I - Surface Tensions and their Variations with Temperature and Impurities - surface tension measurements have been made for gallium using the pendant drop technique and some complex effects ascribed to impurities were observed. In Task 2 - Solutal Convection and Liquid Diffusion Coefficients - the onset of convective and interfacial instabilities during the vertical directional solidification of a binary alloy has been determined theoretically by means of a linear stability analysis. In Task 3 - Determination of Crystal Perfection - a study has been performed on the improvement in resolution of $x$-ray imaging systems for real-time assessment of crystal perfection. In Task 4 - A Thermochemical Study of Corrosion Reactions in Oxide Materials - the system $\mathrm{KFeO}_{2}-\mathrm{Fe}_{2} \mathrm{O}_{3}$ has been examined over portions of the subsolidus region, and the occurrence of a beta alumina structure-type at 85.2 mole percent $\mathrm{Fe}_{2} \mathrm{O}_{3}$ has been observed. In Task 5 - Gravity Effects on Flame Inhibition - a high pressure sampling mass spectrometer facility has been designed and construction on it is nearly completed.

17. KEY WORDS (six to twelve entries; alphabetical order; capitalize only the first letter of the first key word unless a proper name; separated by semicolons)

Convection; corrosive reactions; crystal perfection; flame inhibition; microgravity; solidification; solutal convection; surface tensions
18. AVAIL ABILITY
$\mathrm{X}$ Unlimited

For Official Distribution. Do Not Release to NTIS

Order From Sup. of Doc., U.S. Government Printing Office Washington, D.C. 20402, SD Cat. No. C13

\begin{tabular}{|l|c|}
\hline $\begin{array}{l}\text { 19. SECURITY CLASS } \\
\text { (THIS REPURT) }\end{array}$ & 21. NO. OF PAGES \\
UNCLASSIFIED & 116 \\
\hline $\begin{array}{l}\text { 20. SECURITY CLASS } \\
\text { (THIS PAGE) } \\
\text { UNCLASSIFIED }\end{array}$ & $\begin{array}{l}\text { 22. Price } \\
\$ 6.50\end{array}$ \\
\hline
\end{tabular}

X Order From National Technical Information Service (NTIS) Springfield, Virginia 22151 


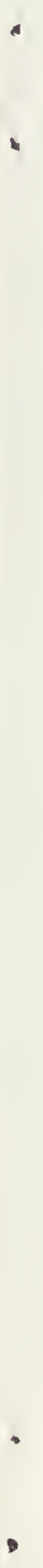
4 
